University of Tennessee Health Science Center UTHSC Digital Commons

\title{
Genetic and Chemical Dissection of BloodBrain Barrier Development in Zebrafish
}

\author{
Robyn Anne Umans \\ University of Tennessee Health Science Center
}

Follow this and additional works at: https://dc.uthsc.edu/dissertations

Part of the Neurosciences Commons

\section{Recommended Citation}

Umans, Robyn Anne, "Genetic and Chemical Dissection of BloodBrain Barrier Development in Zebrafish" (2014). Theses and Dissertations (ETD). Paper 369. http://dx.doi.org/10.21007/etd.cghs.2014.0329.

This Dissertation is brought to you for free and open access by the College of Graduate Health Sciences at UTHSC Digital Commons. It has been accepted for inclusion in Theses and Dissertations (ETD) by an authorized administrator of UTHSC Digital Commons. For more information, please contact jwelch30@uthsc.edu. 


\title{
Genetic and Chemical Dissection of BloodBrain Barrier Development in Zebrafish
}

\begin{abstract}
The blood-brain barrier (BBB) maintains a homeostatic environment as well as prohibits the entrance of xenobiotics into the brain. Because of these qualities, drug delivery is a fundamental challenge for the treatment of many diseases of the central nervous system (CNS). Along with limiting the access of therapeutics into the brain, the BBB is also impaired in CNS pathologies. Understanding the molecular cues that are essential for healthy BBB development and integrity may reveal targets for drug delivery leading to decreased progression or possible treatment of many detrimental CNS diseases. While the central features of the BBB have been accepted, an innovative model has yet to creatively utilize this established knowledge to decipher how the unique signals controlling BBB properties can be optimally targeted. To overcome these scientific barriers, we used zebrafish as a model organism to study BBB development in vivo. We hypothesized that zebrafish could be used to genetically dissect the molecular mechanisms important for the development, function, and maintenance of the BBB. The ultimate goal of this project was to use the zebrafish model for unbiased genetic and small molecule screens. To fulfill these goals, we produced a BBB reporter line, $\mathrm{Tg}$ (glut1b:mCherry)sj1, to characterize the in vivo development of the BBB. We were able to show through live imaging and proof-of-principle experiments, that the processes of CNS angiogenesis and barriergenesis occur concurrently, but do so via independent mechanisms. We then initiated a forward genetic and small molecule screen with our BBB reporter line. From these unbiased, high-throughput approaches, we uncovered several BBB mutants and chemical hits that modulate CNS angiogenesis and the glut $1 \mathrm{~b}$ promoter. We also cloned one of these genetic mutants whereby the mutation was in gpr124, an established gene important for BBB development in mammals. The gpr124 mutant validates that we were able to find genetic modifiers of BBB development in our forward genetic screen. The execution of these screens will not only advance our understanding of BBB development but potentially reveal novel genes and molecules that could be targeted for CNS drug delivery.
\end{abstract}

Document Type

Dissertation

Degree Name

Doctor of Philosophy (PhD)

Program

Biomedical Sciences

Research Advisor

Michael R. Taylor, Ph.D.

Keywords

blood-brain barrier, zebrafish, development, genetic screen, chemical screen

\section{Subject Categories}

Medicine and Health Sciences | Neurosciences

\section{Comments}

One year embargo expired December 2015 
Genetic and Chemical Dissection of Blood-Brain Barrier Development in Zebrafish

\author{
A Dissertation \\ Presented for \\ The Graduate Studies Council \\ The University of Tennessee \\ Health Science Center
}

In Partial Fulfillment

Of the Requirements for the Degree

Doctor of Philosophy

From The University of Tennessee

By

Robyn Anne Umans

December 2014 
Portions of Chapters 1 and 3 @ 2012 by American Society for Clinical Pharmacology and Therapeutics.

Copyright (C) 2014 by Robyn A. Umans.

All rights reserved. 


\section{DEDICATION}

This dissertation is dedicated in loving memory to my grandfather, Bernard Hindes. 


\section{ACKNOWLEDGEMENTS}

I would like to thank Dr. Michael Taylor for his mentorship and the opportunity to perform my dissertation work in his research lab. He transformed genetics from a long-standing topic of struggle to an achievable field of study for me. I am forever grateful for his patience, guidance, and support. He is the type of advisor students hope to find and I was very lucky to have done so. Thank you for being a phenomenal mentor, Michael.

I would like to thank all of my thesis committee members, Drs. Charles Lessman, John Schuetz, Sandra D'Azzo, and Kristin Hamre. I appreciate you volunteering to be a part of my graduate training and your thoughtful input. You all have been so positive and constructive towards completing my degree. I would also like to thank Dr. Lessman for chairing this committee.

I would like to thank my lab members, co-workers, and friends in the department of Chemical Biology and Therapeutics and among St. Jude Children's Research Hospital for all of your assistance and friendship. CBT is a one-of-a-kind environment and I will miss working in such a great department of people. I would like to give special thanks to Drs. Chaithanyarani Parupalli, Sujuan Jia, Wilda Orisme, Hannah Henson, and Jennifer Peters.

I would also like to thank members of my "fish community" for all the help trouble-shooting science and encouragement. Whether it was a "fishue" or not, you all were there for me whenever I needed you. I would like to give a special thank you to my fish sister, Dr. Nikki Glenn.

I would like to thank St Jude Children's Research Hospital and the University of Tennessee Health Science Center for giving me the opportunity to complete my training at top tier institutions. I am also grateful for financial support from the American Lebanese and Syrian Associated Charities. Without their fundraising efforts and monetary contributions to research, none of my research would be possible.

On a personal note, I would like to thank Brenda Royal and Dr. Lora Becker. Brenda, you are the woman who made me fall in love with biology. I honestly wouldn't be where I am today without your influence, love, and support. Lora, you helped foster my love for Neuroscience. Your enthusiasm is contagious and I thank you for encouraging and supporting me to pursue my interest in science.

Last but not least, there is not enough room to adequately thank my friends and family. Thank you for believing in me and supporting me throughout my development as a scientist. You always believed in me when I needed it and never doubted my abilities. I thank my grandparents for always making me feel like a superstar. And of course, thank you mom for your undying love, support, and encouragement. I love you very much. Mom, you are finally allowed to call me Dr. Umans. 


\begin{abstract}
The blood-brain barrier (BBB) maintains a homeostatic environment as well as prohibits the entrance of xenobiotics into the brain. Because of these qualities, drug delivery is a fundamental challenge for the treatment of many diseases of the central nervous system (CNS). Along with limiting the access of therapeutics into the brain, the $\mathrm{BBB}$ is also impaired in CNS pathologies. Understanding the molecular cues that are essential for healthy BBB development and integrity may reveal targets for drug delivery leading to decreased progression or possible treatment of many detrimental CNS diseases. While the central features of the BBB have been accepted, an innovative model has yet to creatively utilize this established knowledge to decipher how the unique signals controlling BBB properties can be optimally targeted. To overcome these scientific barriers, we used zebrafish as a model organism to study BBB development in vivo. We hypothesized that zebrafish could be used to genetically dissect the molecular mechanisms important for the development, function, and maintenance of the BBB. The ultimate goal of this project was to use the zebrafish model for unbiased genetic and small molecule screens. To fulfill these goals, we produced a BBB reporter line, $\operatorname{Tg}$ (glut lb:mCherry) ${ }^{s j 1}$, to characterize the in vivo development of the BBB. We were able to show through live imaging and proof-of-principle experiments, that the processes of CNS angiogenesis and barriergenesis occur concurrently, but do so via independent mechanisms. We then initiated a forward genetic and small molecule screen with our $\mathrm{BBB}$ reporter line. From these unbiased, high-throughput approaches, we uncovered several BBB mutants and chemical hits that modulate CNS angiogenesis and the glut $1 b$ promoter. We also cloned one of these genetic mutants whereby the mutation was in gpr 124, an established gene important for BBB development in mammals. The gpr 124 mutant validates that we were able to find genetic modifiers of BBB development in our forward genetic screen. The execution of these screens will not only advance our understanding of BBB development but potentially reveal novel genes and molecules that could be targeted for CNS drug delivery.
\end{abstract}




\section{TABLE OF CONTENTS}

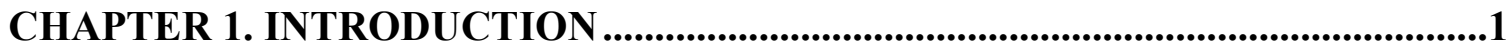

Background of the Blood-Brain Barrier ..................................................................... 1

Blood-Brain Barrier in Health and Disease .......................................................... 1

Drug Delivery Across the Blood-Brain Barrier ........................................................2

Blood-Brain Barrier Structure ...............................................................................2

Molecular Mechanisms that Regulate the Blood-Brain Barrier.................................4

Models Used to Study the Blood-Brain Barrier.........................................................6

Different Models to Study the Blood-Brain Barrier ..................................................6

Zebrafish as a Model Organism .........................................................................

Zebrafish as a Model to Study Blood-Brain Barrier Development ...........................9

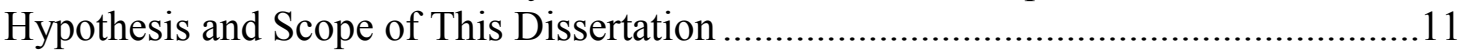

\section{CHAPTER 2. GENERATION OF A BLOOD-BRAIN BARRIER REPORTER}

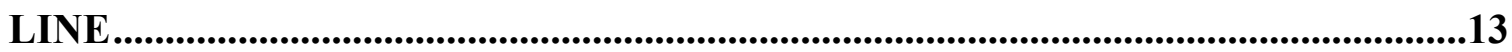

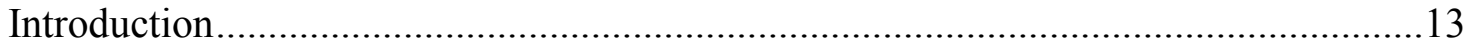

Transgenics Used to Study the Blood-Brain Barrier ............................................... 13

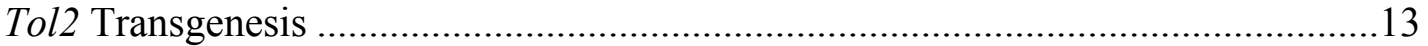

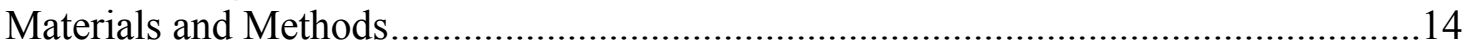

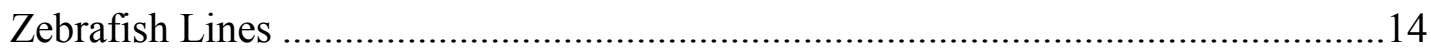

Tol2 Transgenesis and Construct Microinjection ................................................. 14

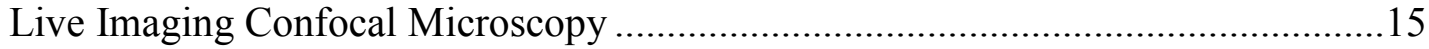

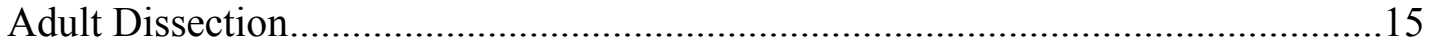

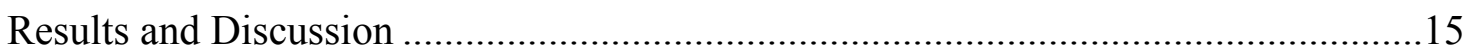

Selecting a BBB-Promoter, Glucose Transporter 1 ................................................ 15

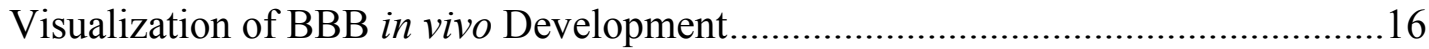

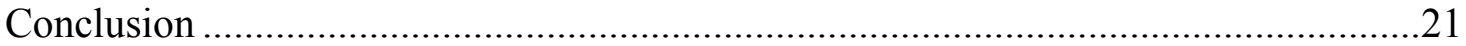

\section{CHAPTER 3. CHARACTERIZATION OF THE BLOOD-BRAIN BARRIER REPORTER LINE........................................................................................................23}

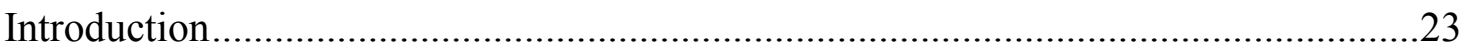

Characteristic Properties of the BBB ....................................................................23

Methods for Testing the Function and Structure of the BBB ...............................23

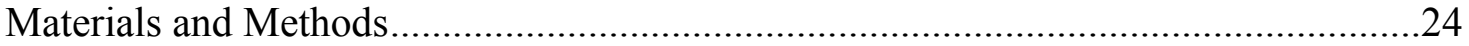

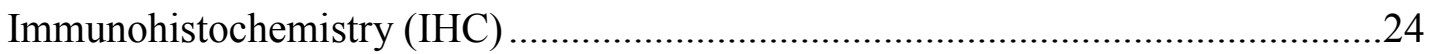

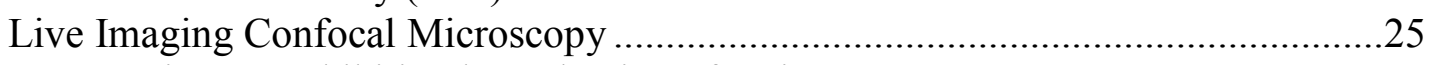

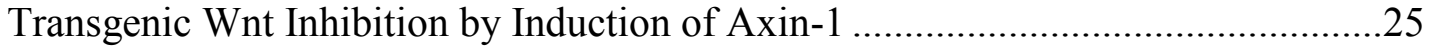

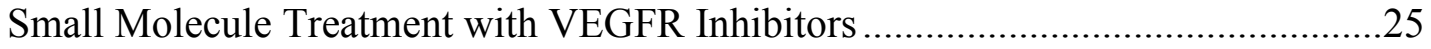

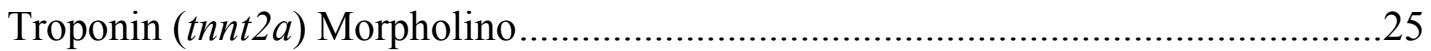

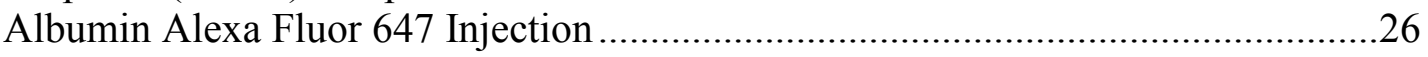

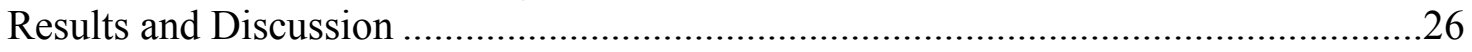

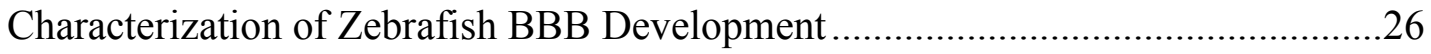

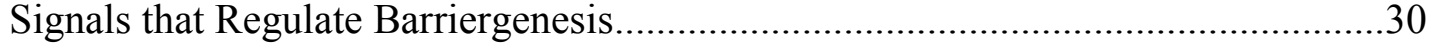

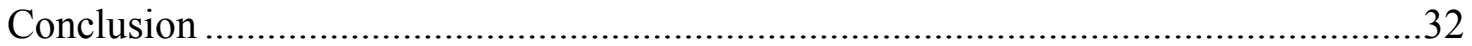




\section{CHAPTER 4. A FORWARD GENETIC SCREEN TO UNCOVER BLOOD-

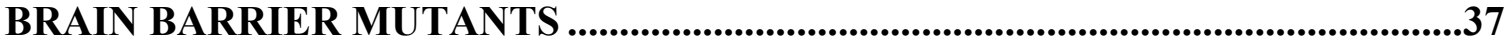

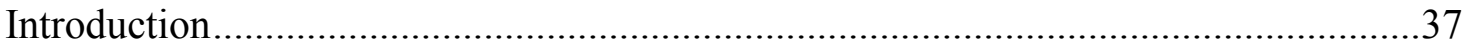

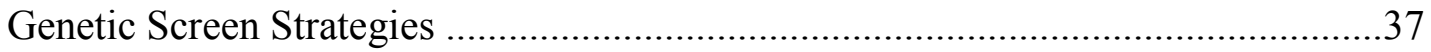

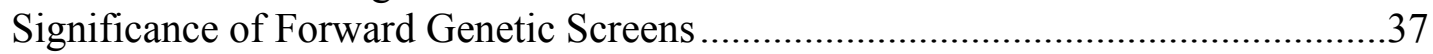

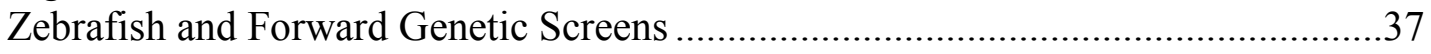

Treatment and Breeding Scheme in Zebrafish Forward Genetic Screens .................38

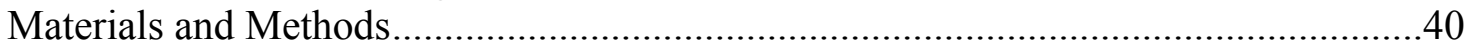

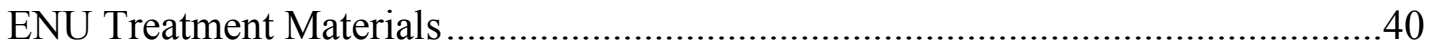

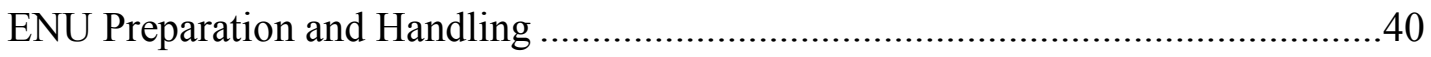

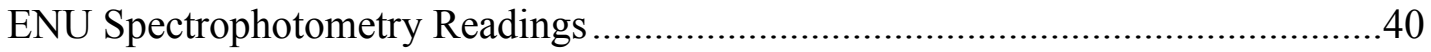

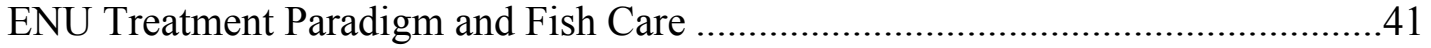

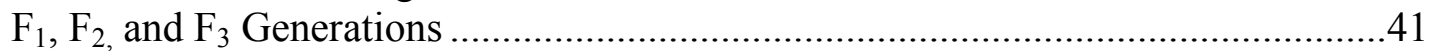

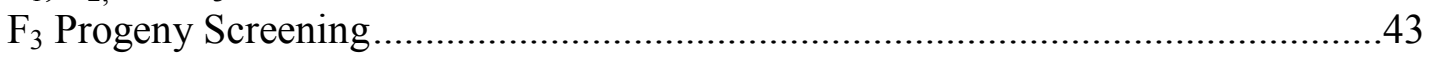

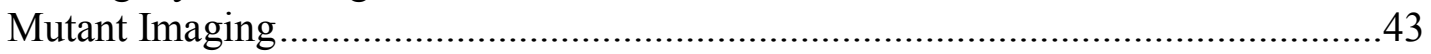

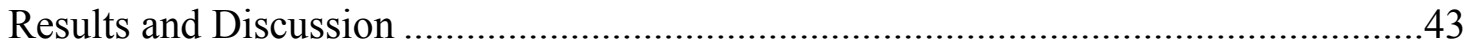

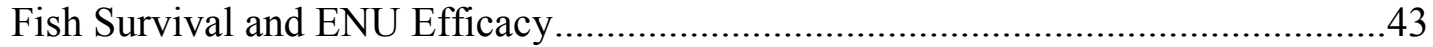

BBB Mutants Discovered from Our Forward Genetic Screen .................................44

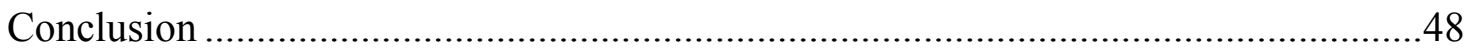

\section{CHAPTER 5. CLONING AND CHARACTERIZATION OF THE FIRST}

ZEBRAFISH BLOOD-BRAIN BARRIER MUTANT, 22.6....................................50

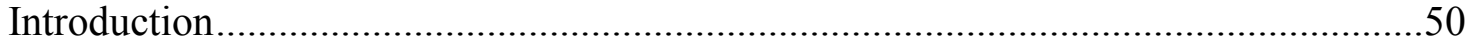

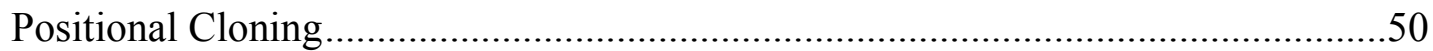

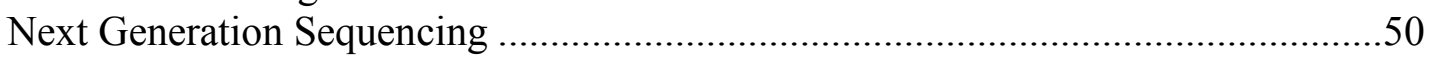

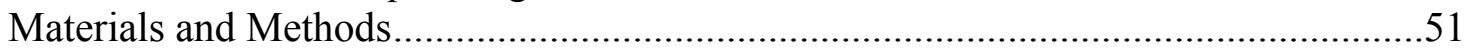

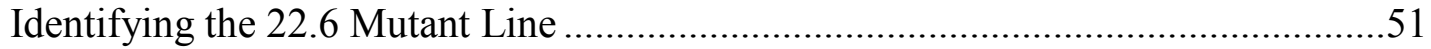

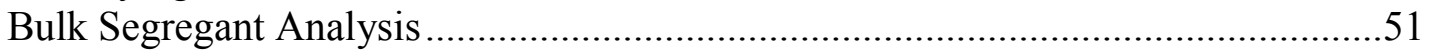

Exome Sequencing..................................................................................52

Polymerase Chain Reaction (PCR) and Sequencing of Candidate 22.6 Mutations...52

Reverse Transcriptase Polymerase Chain Reaction (RT-PCR) of gpr 124 ...............53

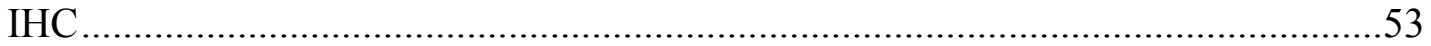

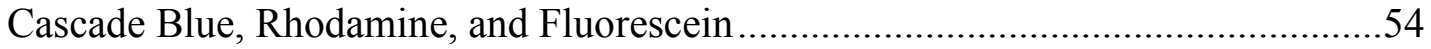

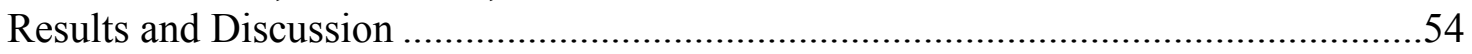

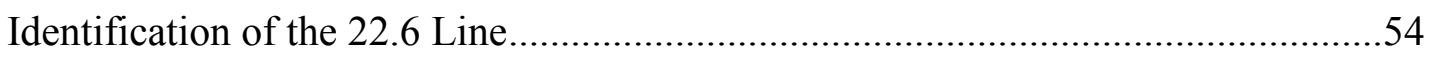

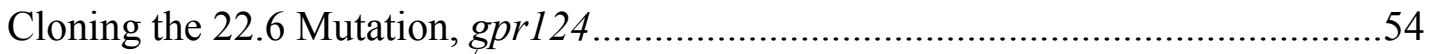

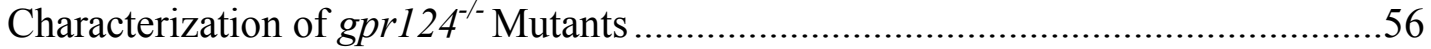

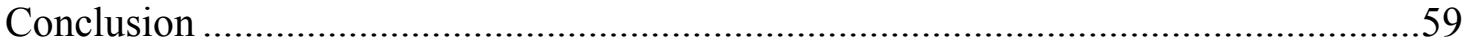

\section{CHAPTER 6. A CHEMICAL SCREEN TO IDENTIFY MODULATORS OF} BLOOD- BRAIN BARRIER DEVELPOMENT ................................................62

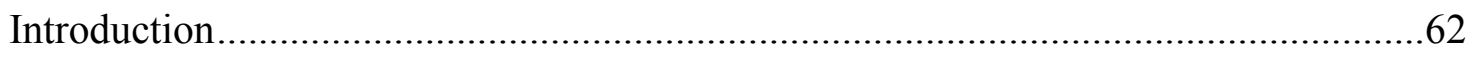

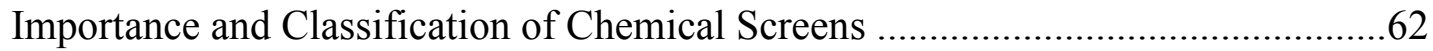

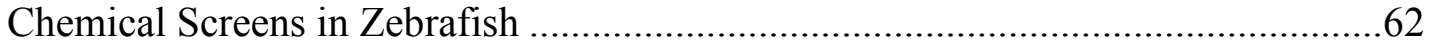

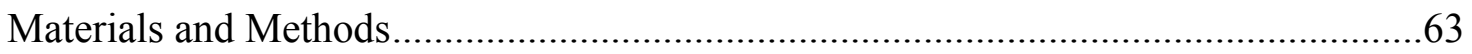


Chemical Libraries Tested ...........................................................................6 63

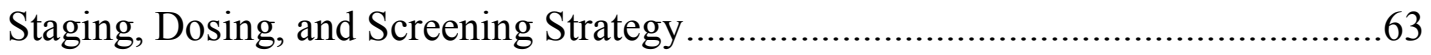

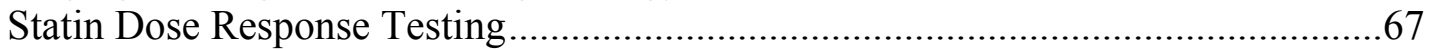

Tunicamycin, Forskolin, and GBR13069 Treatment..........................................67

Dolichol Kinase (dolk) Morpholino Injections and RT-PCR ................................67

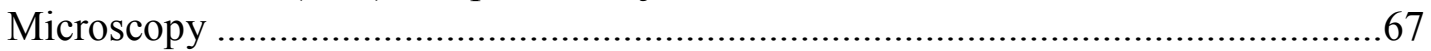

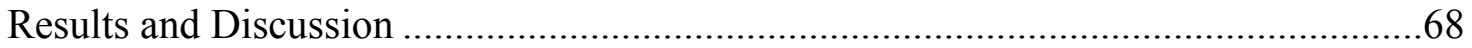

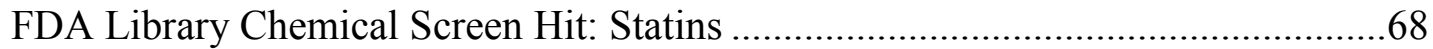

ENZO Wnt and Autophagy Library Hit: Forskolin ............................................. 73

ENZO Neurotransmitter Library Hit: GBR13069 ............................................ 78

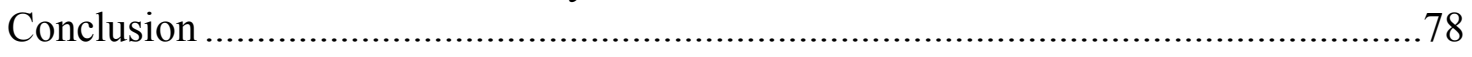

CHAPTER 7. SUMMARY AND CONCLUSIONS.......................................................82

LIST OF REFERENCES ......................................................................................................88

VITA 


\section{LIST OF TABLES}

Table 4-1. Observation of mutant lines over the course of development.....................46

Table 5-1. Candidate mutations for the 22.6 line ..........................................................56 


\section{LIST OF FIGURES}

Figure 1-1. The neurovascular unit illustrating the various cell types of the bloodbrain barrier.

Figure 1-2. Zebrafish are a suitable model organism for studying developmental biological processes

Figure 1-3. Comparative timeline of events between zebrafish and mammals during blood-brain barrier development. 10

Figure 2-1. Blood-brain barrier construct injection into $\operatorname{Tg}(f l i l a: E G F P)^{y 1}$ embryos ......17

Figure 2-2. Transient expression of glut 1b:mCherry in flila:EGFP brain endothelium

Figure 2-3. glut $1 b$ is expressed in brain endothelial cells and not in circumventricular organs

Figure 2-4. glut 1b:mCherry is maintained in adult brain endothelial cells as well as reproductive tissues .20

Figure 2-5. Angiogenesis and barriergenesis occur simultaneously .22

Figure 3-1. Zebrafish brain endothelial cells express Claudin5 and Glut1 during development

Figure 3-2. Zebrafish brain endothelial cells express Mdr1 during development. .28

Figure 3-3. Newly formed vessels are impermeable to albumin .29

Figure 3-4. Levels of Wnt inhibition are permissive for angiogenesis but not barriergenesis

Figure 3-5. Angiogenesis is not required for barriergenesis to occur.

Figure 3-6. Shh and BMP signaling is not required for glut $1 b$ expression in the zebrafish blood-brain barrier

Figure 3-7. Circulation is not required for glut $1 b$ expression in the zebrafish bloodbrain barrier.

Figure 4-1. Breeding scheme for a zebrafish $\mathrm{F}_{3}$ forward genetic screen. . .39

Figure 4-2. ENU treatment paradigm for male $T g($ glut lb:mCherry;flila:EGFP $)$..........42

Figure 4-3. Effectiveness of $3 \mathrm{mM} \mathrm{ENU}$ after the $1^{\text {st }}$ round of ENU treatment. . .45 
Figure 4-4. CNS angiogenesis mutants identified from the forward genetic screen .......47

Figure 4-5. $\operatorname{Tg}($ glut $1 b: m$ Cherry) mutant and control sibling identified from the forward genetic screen

Figure 5-1. Workflow comparison of bulk segregant analysis and exome sequencing for cloning zebrafish mutants from a forward genetic screen ......................55

Figure 5-2. Sequencing larvae for the 22.6 candidate gene mutations

Figure 5-3. Immunohistochemical analysis of BBB markers Glut1 and Claudin5 in 22.6 mutants and wild-type siblings 58

Figure 5-4. 22.6/TL mutants appear "leaky" after IV injections with Cascade Blue .60

Figure 5-5. The 22.6/TL mutant blood-brain barrier is leaky with various sized tracers

Figure 6-1. Zebrafish embryos are amenable for high-content small molecule screening......

Figure 6-2. Weekly workflow for the blood-brain barrier chemical screen .66

Figure 6-3. Statins induce cerebral hemorrhage and reduce CNS angiogenesis in the developing zebrafish

Figure 6-4. Zebrafish as a predictive indicator of blood-brain barrier penetration of statins.

Figure 6-5. Experimental design for a developmental time course with Atorvastatin and Rosuvastatin treatments....

Figure 6-6. Statin treatment prior to zygotic transcription disrupts CNS angiogenesis ..72

Figure 6-7. Statin treatment from 24 or $48 \mathrm{hpf}$ to $72 \mathrm{hpf}$ disrupts CNS angiogenesis ....74

Figure 6-8. Tunicamycin causes a similar disruption to CNS vessels as does statin treatment.

Figure 6-9. dolk is maternally derived and disrupts CNS angiogenesis when targeted with morpholinos .76

Figure 6-10. dolk morphants have normal peripheral angiogenesis .77

Figure 6-11. Forskolin was a hit from both ENZO Wnt and Autophagy libraries .79

Figure 6-12. GBR13069 dose response shows a decrease in Tg (glut1b:mCherry) in the hindbrain of the developing zebrafish 


\section{LIST OF ABBREVIATIONS}

$\begin{array}{ll}\text { ABC } & \text { ATP-Binding Cassette } \\ \text { adra2b } & \text { Alpha-2B Adrenoceptor } \\ \text { BBB } & \text { Blood-Brain Barrier } \\ \text { BEC } & \text { Brain Endothelial Cell } \\ \text { BMP } & \text { Bone Morphogenic Protein } \\ \text { bp } & \text { Base Pair } \\ \text { BSA } & \text { Bulk Segregant Analysis } \\ \text { cAMP } & \text { Cyclic Adenosine Monophosphate } \\ \text { cDNA } & \text { Complementary DNA } \\ \text { CNS } & \text { Central Nervous System } \\ \text { CSF } & \text { Cerebral Spinal Fluid } \\ \text { CtA } & \text { Central Arteries } \\ \text { Da } & \text { Dalton } \\ \text { DMSO } & \text { Dimethyl Sulfoxide } \\ \text { DNA } & \text { Deoxyribonucleic Acid } \\ \text { DOLK } & \text { Dolichol Kinase } \\ \text { dpf } & \text { Days Post Fertilization } \\ \text { EC } 50 & \text { Half Maximal Effective Concentration } \\ \text { EGFP } & \text { Enhanced Green Fluorescent Protein } \\ \text { ENU } & \text { N-Ethyl-N-Nitrosourea } \\ \text { FDA } & \text { Food and Drug Administration } \\ \text { fli1a } & \text { Friend Leukemia Virus Integration 1a } \\ \text { GLUT1 } & \text { Glucose Transporter 1 } \\ \text { GPR124 } & \text { G-Coupled Protein Receptor 124 } \\ \text { hpf } & \text { Hours Post Fertilization } \\ \text { IHC } & \text { Immunohistochemistry } \\ \text { IV } & \text { Intravenous } \\ \text { kb } & \text { Kilo Base } \\ \text { MDR1 } & \text { Multi Drug Resistant Protein 1 } \\ \text { MGH } & \text { Massachusetts General Hospital } \\ \text { mRNA } & \text { Messenger Ribonucleic Acid } \\ \text { NGS } & \text { Next Generation Sequencing } \\ \text { NT } & \text { Neurotransmitter } \\ \text { NVU } & \text { Neurovascular Unit } \\ \text { OD } & \text { Optical Density } \\ \text { PBS } & \text { Phosphate Buffered Saline } \\ \text { PCR } & \text { Polymerase Chain Reaction } \\ \text { PHBC } & \text { Primordial Hindbrain Channel } \\ \text { PTU } & \text { N-Phenylthiourea } \\ \text { RT-PCR } & \text { Reverse Transcriptase Polymerase Chain Reaction } \\ \text { Shh } & \text { Sonic Hedgehog } \\ \text { TALENS } & \text { Transcription Activator-like Effector Nucleases } \\ \text { TEER } & \text { Transendothelial Electrical Resistance } \\ & \\ \text { TH } & \end{array}$




$\begin{array}{ll}\text { Tg } & \text { Transgenic } \\ \text { TILLING } & \text { Targeting Induced Local Lesions In Genomes } \\ \text { TL } & \text { Tubingen Longfin } \\ \text { tnnt2a } & \text { Cardiac Troponin T2A } \\ \text { VEGF } & \text { Vascular Endothelial Growth Factor } \\ \text { WES } & \text { Whole Exome Sequencing } \\ \text { WGS } & \text { Whole Genome Sequencing }\end{array}$




\section{CHAPTER 1. INTRODUCTION*}

\section{Background of the Blood-Brain Barrier}

The blood-brain barrier (BBB) is a unique structure crucial for maintaining central nervous system (CNS) homeostasis. The physical and chemical properties of brain endothelial cells (BECs) regulate the access of compounds from blood to brain and brain to blood. While it is necessary for BECs to retain these properties, this physiological system hinders the efficacious delivery of therapeutics into the CNS. Even though the BBB was discovered over a century ago, molecular mechanisms and interacting cell types necessary for BBB function and integrity have only recently become uncovered. The field of BBB biology could benefit from innovatively utilizing a model system to dissect elements crucial for barrier properties. These molecular pathways could be modulated to aid in drug delivery and prevent disease progression.

\section{Blood-Brain Barrier in Health and Disease}

The BBB is one of three blood-CNS barriers playing a major role in CNS health and disease. The BBB is endothelial cell based unlike the epithelial cell based choroid plexus and arachnoid barriers that exist between the cerebral spinal fluid (CSF) and blood $^{1}$. Each of these barriers exhibits a unique physical and molecular signature in order to protect the CNS. While all three barriers contribute to a healthy CNS, studies on the $\mathrm{BBB}$ have been more prominent in the past few decades.

A healthy neurovascular structure is necessary for neuronal function as the brain is a highly metabolic organ, requiring $20 \%$ of cardiac output ${ }^{2}$. The BBB maintains the proper environment crucial for neuronal activity, ionic homeostasis, and prohibits harmful xenobiotics from entering the brain parenchyma ${ }^{2}$. These functions provide the brain with a chemical safeguard and prevent neural complications. However, it is unknown whether alteration of the BBB is a cause or effect of disease states. The BBB is disrupted in many CNS pathologies such as Alzheimer's disease, Parkinson's disease, Multiple Sclerosis, AIDS Dementia, stroke, and epilepsy ${ }^{2,3}$. Reduction in blood flow in the aging brain may lead to a change in cerebral protein synthesis, action potential firing, and electrolyte dysbalance ${ }^{2}$. In a recent study by Bell et al. (2010), it was suggested that BBB break down exists before disease progression ${ }^{4}$. Their mouse model of pericyte loss in the adult brain produced vascular defects and the subsequent initiation of secondary neurodegenerative changes ${ }^{4}$. The health and function of the BBB not only plays a role in the pathophysiology of CNS disease, but also in the treatment of conditions such as CNS tumors.

*Modified with permission. Umans, R. A. \& Taylor, M. R. Zebrafish as a model to study drug transporters at the blood-brain barrier. Clin Pharmacol Ther 92, 567-570, doi:10.1038/clpt.2012.168 (2012). 


\section{Drug Delivery Across the Blood-Brain Barrier}

The protection the BBB offers the brain simultaneously prohibits the access of most therapeutics from entering the CNS. In fact, around $100 \%$ of large molecules and $98 \%$ of small molecules are excluded from entering the brain ${ }^{5}$. CNS drug penetration is dependent on drug lipid solubility as well as a lower molecular weight ${ }^{6}$. These therapeutic limitations are imposed by a lack of fenestrations, presence of tight junctions, and expression of multi-drug resistance efflux pumps on BECs ${ }^{7}$. The main multi-drug resistance protein found in the BBB, P-glycoprotein, was discovered due to its overexpression in cancer cells and consequently was found to be one of the major players for drug resistance at the $\mathrm{BBB}^{8,9}$. P-glycoprotein, as well as other members of the multi-drug resistance protein families, excludes various anti-cancer, anti-psychotic, anti-epileptic, and anti-HIV drugs ${ }^{7}$. While it is necessary for the BBB to regulate the passage of compounds into the brain, this access is not biased towards compounds that could otherwise medicate CNS diseases.

To more effectively deliver therapeutics into the CNS, various approaches and targets have been established. Biomedical and chemical tactics are commonly employed to more effectively deliver drugs into the CNS. Receptor-mediated endocytosis of biodegradable nanoparticles coated in various drugs successfully traverse the barrier in vitro and in vivo ${ }^{10}$. Multi-drug resistance transporters and other endogenous mechanisms may be targeted for CNS drug delivery although a good structure-activity relationship is needed for effective drug design. Drugs can be designed as transporter substrates or conversely inhibit transporters to allow more uptake of the desired therapeutic ${ }^{11,12}$. Aside from biochemical approaches, physical techniques have also been investigated for opening up the BBB. MRI- targeted ultrasound has been shown to breakdown the paracellular barrier between BECs in a focused area ${ }^{13}$. MRI-induced BBB opening allowed the delivery of antibodies in the CNS yet physical damage to tissue is an artifact of this process ${ }^{14}$. More invasive methods exist, such as intra-cerebro-infusion and biodegradable implants aiming to physically break down the barrier and locally deliver therapy ${ }^{15}$. However, it is less likely patients would prefer a more crude, surgical technique, especially since drugs diffuse better into the blood versus the brain and multiple implants may need to be transplanted in order to have an effective treatment volume $^{6,16}$. Drug delivery through the olfactory system is also a route for CNS drug delivery. Molecules move into the brain through the nose by entering the olfactory CSF, very similarly to intra-cerebro-infusions, yet less invasive ${ }^{16}$. Several kinds of compounds such as sulfonamides, insulin, and progesterone have entered the CNS via nasal delivery but measures need to be taken as to not allow viruses or bacteria to enter the CNS through this course ${ }^{11}$.

\section{Blood-Brain Barrier Structure}

The BBB was first described over a century ago by Paul Ehrlich as he injected various water-soluble dyes into circulation and witnessed their exclusion from the brain and spinal cord ${ }^{17,18}$. Edwin Goldmann, Ehrlich's student, further characterized these 
observations through which he witnessed the retention of trypan blue in the CSF and not the periphery when injected directly into the $\mathrm{CSF}^{19}$. The term "blood-brain barrier" was however created by Max Lewandowksy whereby he discovered neurotoxic agents only had a harmful effect after direct injection into the brain ${ }^{20}$. These pioneering experiments laid the ground work for the presence of a functional barrier but still posed the question of how and why it developed. In fact, it wasn't until 1969 that Brightman and Reese first localized tight junction proteins in between BECs, identifying the structure that was responsible for controlling passive diffusion of different molecules ${ }^{21}$.

Since then, it has been widely established that the selectivity of the barrier is due in part to both its physical and chemical barriers ${ }^{2}$. Tight junctions, proteins anchored between endothelial cells, are important for establishing the paracellular regulation between cells. The main classes of tight junction proteins are Occludins and Claudins, tethered between cells by junction adhesion molecules. When absent, loss of Claudin 5 causes a size-selective loosening of the $\mathrm{BBB}^{22}$. In addition to a physical blockade, these proteins mediate the restriction of small molecule movement through trans-endothelial electrical resistance (TEER). In fact, TEER in the periphery is reported to be fifty times lower than between BECs ${ }^{23}$. Transporters, both carrier mediated and active efflux, regulate the access of particular nutrients as well as prohibit the entry of xenobiotics into the brain ${ }^{2}$. Throughout the CNS, transporters traffic a diverse range of compounds. Several classes of transporters exist along blood-CNS barriers to direct the proper distribution of drugs, xenobiotics, and nutrients.

While different transporters are isolated to specific locations among the bloodCNS barriers, transporters of the BBB are perhaps the best characterized. With over 40 individual members of the ATP-binding cassette ( $\mathrm{ABC}$ ) family, $\mathrm{ABC}$ transporters are the most widely studied drug transporters at the BBB. Of the $\mathrm{ABC}$ transporters, $\mathrm{ABCB} 1$, also known as Multidrug Resistance Protein 1 (MDR1) and P-glycoprotein, plays a prominent function in excluding compounds from the CNS. Other $\mathrm{ABC}$ family members such as BCRP (ABCG2) and MRP4 (ABCC4) are also highly expressed at the BBB and play important roles in drug transport ${ }^{7}$. Knockout mice of the MDR1 mouse ortholog, $M d r l a$, revealed higher sensitivities to both neurotoxic Ivermectin and chemotherapeutic Vinblastine compared to wild-type littermates, indicating a protective activity of MDR1a at the mouse $\mathrm{BBB}^{24}$. Since its discovery, many groups have attempted to modulate MDR1 function as a means to alter therapeutic bioavailability and to overcome multidrug resistance.

In addition to drug efflux transporters, BECs express many anionic and carrier mediated systems to deliver the proper nutrition to the CNS. Organic anion transporters mediate the distribution of neurotransmitter (NT) metabolites as well as other anions throughout the $\mathrm{CNS}^{25}$. Amino acid transporters help deliver essential amino acids as well as regulate potential amino acid neurotoxicity in the $\mathrm{CNS}^{2}$. Glucose Transporter 1 (GLUT1), a solute carrier, is vital for proper brain function since glucose is impermeant to the BBB and the brain uses approximately $20 \%$ of whole-body glucose ${ }^{26}$. GLUT1 is highly expressed in BECs in order to provide energy to the brain and is often used as a 
marker for BBB localization and function ${ }^{27}$. GLUT1 is also one of the earliest BBB markers as it is expressed in BECs early in brain development ${ }^{28}$.

Furthermore, it is still widely debated in the field of brain barrier biology whether angiogenesis and barriergenesis occur simultaneously or in a two-step process ${ }^{1}$. The first hypothesis suggests that barriergenesis occurs as soon as angiogenesis takes place in the brain. The second hypothesis suggests that angiogenesis of leaky, immature vessels occurs in the brain followed later by barriergenesis whereby BECs adapt the BBB phenotype. In order to advance the field of BBB biology and answer these unsettled mysteries about barriergenesis, innovative strategies must be applied to study BBB development in vivo and find novel signals controlling this process.

In addition to the structural components of the BBB, different cell types associate with BECs to fully establish what is deemed the neurovascular unit (NVU)

(Figure 1-1) $^{23}$. The NVU is a physiological arrangement of BECs, neurons, astrocytes, pericytes, and microglia ${ }^{2}$. Pericytes, a kind of smooth muscle cell, develop during initial CNS angiogenesis in the rat at E12 and E11.5 in mouse before astrocytic endfeet ensheath BECs post-natally ${ }^{27} 29$. Initially, astrocytes were speculated to be the cell type important for barriergenesis as astrocytes relay cell non-autonomous signals important for barrier differentiation and function ${ }^{30}$. It is now understood that pericytes are important for barrier function in mice at a time when astrocytes have not yet developed ${ }^{27}$. Microglia, the primary immune cell of the CNS, survey their environment and change into phagocytic cells when activated ${ }^{2}$. Many studies are now aimed at deciphering the molecular cues that ensue between the cell types of the NVU as a means to target them therapeutically.

\section{Molecular Mechanisms that Regulate the Blood-Brain Barrier}

The signals important for BBB development are still not completely understood. Although much remains unknown about signaling during BBB development, certain pathways and molecules essential for barriergenesis have recently been uncovered. The cell types of the NVU relay cell-autonomous and non-autonomous signals to one another in order to create a functional BBB 31323334,35 . Daneman et al. (2009) demonstrated that Wnt signaling is important for CNS angiogenesis but not peripheral angiogenesis, suggesting that $\mathrm{Wnt}$ is included in a distinct signaling profile for BBB development ${ }^{36}$. GCoupled Protein Receptor 124 (GPR124), an orphan g-coupled protein receptor upregulated in colorectal cancer, is also crucial for proper BBB formation ${ }^{34,35}$. Interestingly, both Wnt and GPR124 mutant mice have similar phenotypes, including embryonic lethality and abnormal CNS angiogenesis in the forebrain and spinal cord ${ }^{35,36}$. Additionally, Sonic hedgehog (Shh) has been suggested to promote barrier integrity through secretion of Shh by astrocytes ${ }^{33}$. These findings advance the understanding of signals important in BBB development, but do so using a candidate gene approach. While effective, such methods may shed bias on results, especially since some of these pathways are already established to be important for brain development ${ }^{37,38}$. 


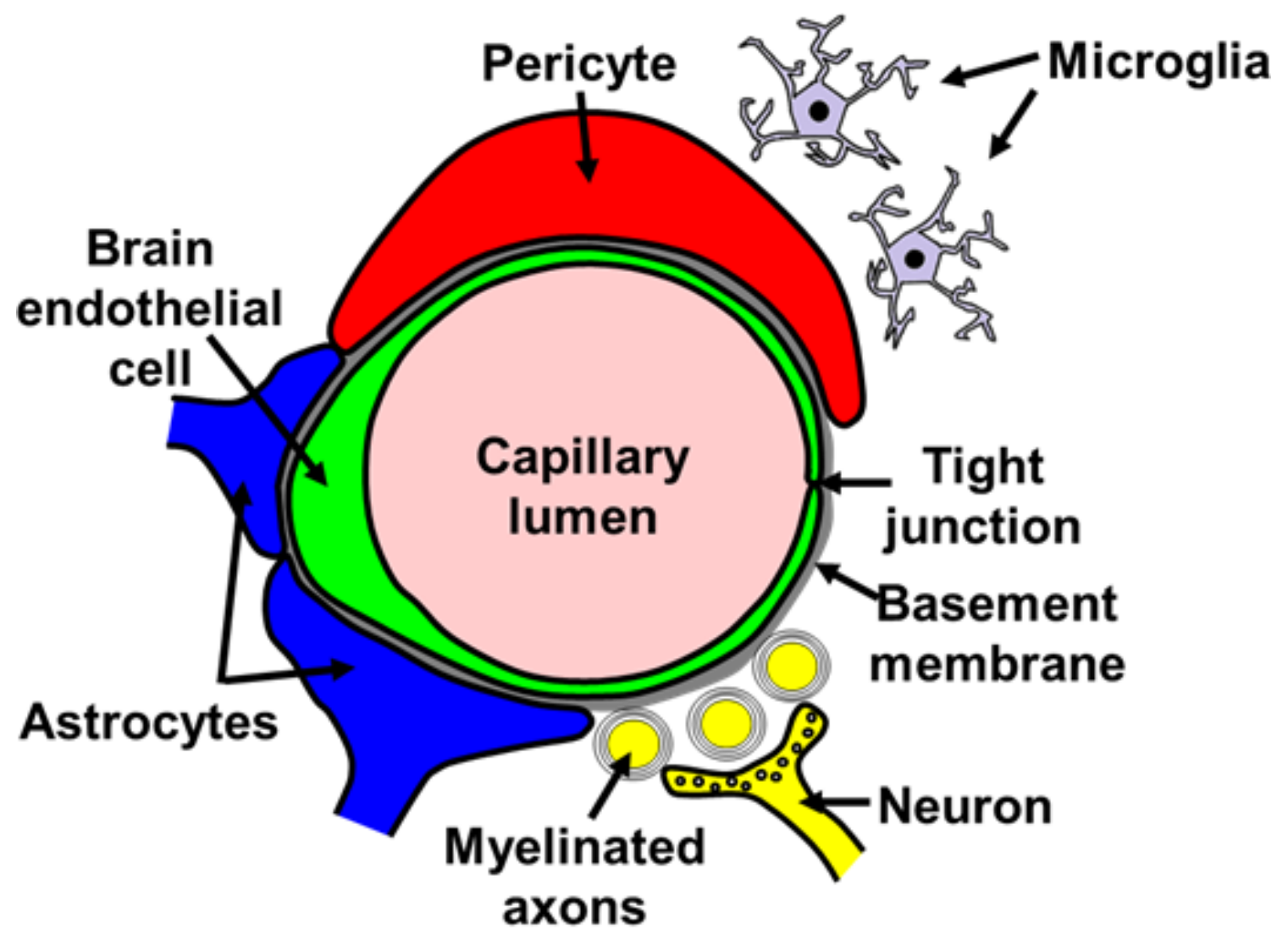

Figure 1-1. The neurovascular unit illustrating the various cell types of the bloodbrain barrier

Brain endothelial cells interact with pericytes, astrocytes, neurons and microglia to maintain CNS homeostasis. 
In recent years, the molecular signatures of BECs have been investigated in order to find specific targets at the BBB. Daneman et al. (2010) compared the transcriptional profile of isolated BECs compared to vessels found in the liver and lung ${ }^{39}$. From this study, a mouse BBB transcriptome was developed as a resource for further understanding what signals are enriched in this brain barrier. Ben-Zvi et al. (2014) recapitulated a similar assay, isolating $M f_{S} d 2 a$ as a BBB enriched gene ${ }^{40}$. Knockout of $M f_{s} d 2 a$ resulted in normal vascular patterning but a leakier $\mathrm{BBB}^{40}$. Genes such as $M f_{s} d 2 a$ and ones found in the mouse BBB transcriptome could ideally be targeted for CNS drug delivery.

\section{Models Used to Study the Blood-Brain Barrier}

\section{Different Models to Study the Blood-Brain Barrier}

A variety of models are used to study BBB function and its molecular mechanisms. Just as Ehrlich used several animal models to assess BBB function over a century ago, a current array of models exists to study BBB development and maturation. Among the models used are avian, rodent, insect, and tissue culture systems. Avian studies first suggested there are signals in the brain milieu responsible for BBB development ${ }^{32}$. Stewart and Wiley (1981) performed transplantation studies revealing peripheral vessels could adopt BBB features when they vascularized CNS transplanted tissue, suggesting a difference between the microenvironment of the periphery of the body and the brain ${ }^{32}$. Rodent models, which are most popularly used, have helped identify the proteins important to the physical and chemical barriers of the BBB, the cell types that are important in the NVU, the consequences of particular cell type loss, the expression patterns of BBB markers, and signals that are communicated between these cell types ${ }^{21,24,27,28,30,31,33-36,39-41}$. Armulik et al. (2010) demonstrated that pericytes in the rodent $\mathrm{BBB}$ are crucial for proper regulation by maintaining BBB function, BBB-specific gene expression, and astrocytic end-feet contact ${ }^{41}$. This significant finding also contradicted previous theories that suggested that astrocytes regulate BBB formation even though pericytes develop before astrocytes ${ }^{1,30}$. Furthermore, insect models possess technical advantages as they are used easily and rapidly in molecular biology ${ }^{42}$. Mayer et al. (2009) demonstrated an evolutionary conservation of the BBB in Drosophila melanogaster and further demonstrated the use of fruit flies for studying BBB physiology ${ }^{43}$. Tissue culture systems are utilized independently or in combination with rodent studies to examine the importance of cellular interactions and gene expression in the $\mathrm{BBB}^{44}$. Because the various cell types of the NVU have now been established, models have been developed utilizing more than two cell types to more accurately recapitulate BBB properties in vitro. Lippmann et al. (2011) co-cultured differentiated rat neural progenitor cells with rat brain microvascular endothelial cells to more effectively elevate and recapitulate $\mathrm{BBB}$ properties in endothelial cells compared to the more tediously acquired primary rat astrocyte cell co-culture models ${ }^{45}$. It is important to consider the strengths and weaknesses of each of these models when asking questions about BBB development. In recent years, the zebrafish (Danio rerio) has emerged as a suitable model organism for studying blood-CNS barriers. 


\section{Zebrafish as a Model Organism}

Zebrafish are genetically tractable vertebrates with high fecundity. Offspring mature rapidly, outside of the mother, allowing for manipulation early in development. Within 26 hours post-fertilization (hpf), zebrafish embryos already have developing eyes, a brain, heartbeat and circulation. Their transparent nature also allows for the visualization of these early processes in real time (Figure 1-2), making zebrafish an excellent model for developmental biology studies. Zebrafish are a member of the teleosts, a class of fish that underwent a genome duplication event during the course of evolution but still have a similar number of chromosomes to humans ${ }^{46}$. Due to conservation in genomic structure, vertebrate body plan, and systemic biology, zebrafish are popularly used in genetic screens to uncover genes orthologous to human ${ }^{47}$.

Since the first small-scale mutagenesis screen in the early 1990s, zebrafish have been exploited for large-scale mutagenesis screens, whole-genome sequencing, positional cloning, insertional mutagenesis, germline transgenesis, and modeling human disease ${ }^{47}$. Forward genetic screens surged during the 1990s in Boston, Massachusetts and Tübingen, Germany, initially uncovering genes essential for embryogenesis ${ }^{48,49}$. This approach identifies point mutations randomly arrayed throughout the genome based on phenotypic screening. Forward genetic screens have identified mutants in signaling pathways involved in various aspects of physiology such as vision, tumorigenesis, and angiogenesis $^{50-52}$. While it is more cost effective and easier to produce large zebrafish clutches for forward genetics, zebrafish are still used for reverse genetic approaches just as rodents are. With the advancement of technology, reverse genetics in fish is possible with a variety of techniques such as morpholinos, Targeting Induced Local Lesions in Genomes (TILLING), Zinc finger nucleases, transcription activator-like effector nucleases (TALENS), and CRISPR/Cas9 systems $^{53-55}$. Human mutations have also been introduced in the zebrafish that cause the same diseases derived in human. Mutations in $B R A F(V 600 E)$ from human melanoma introduced into the fish also caused the disease and identified molecular regulators to accelerate the malignancy, suggesting a conservation of genes between zebrafish and mammals ${ }^{56}$. Zebrafish are not only ideal for these genetic screens but for chemical assays as well.

Chemical screens are targeted, high-throughput platforms that have been extensively validated with the zebrafish model system ${ }^{57}$. These assays provide a powerful tool for therapeutic discovery as compound libraries are administered to multiple, whole vertebrates in a multi-well format. The power of zebrafish genetics is also commonly utilized for chemical biology studies. Chemical libraries may be applied to genetic mutants to rescue disease-related phenotypes as well as validate structureactivity relationships ${ }^{57}$. Previous zebrafish chemical screens have identified compounds that affect signaling pathways such as Bone Morphogenic Protein (BMP), cell cycle progression, and cancer angiogenesis ${ }^{58-60}$. 


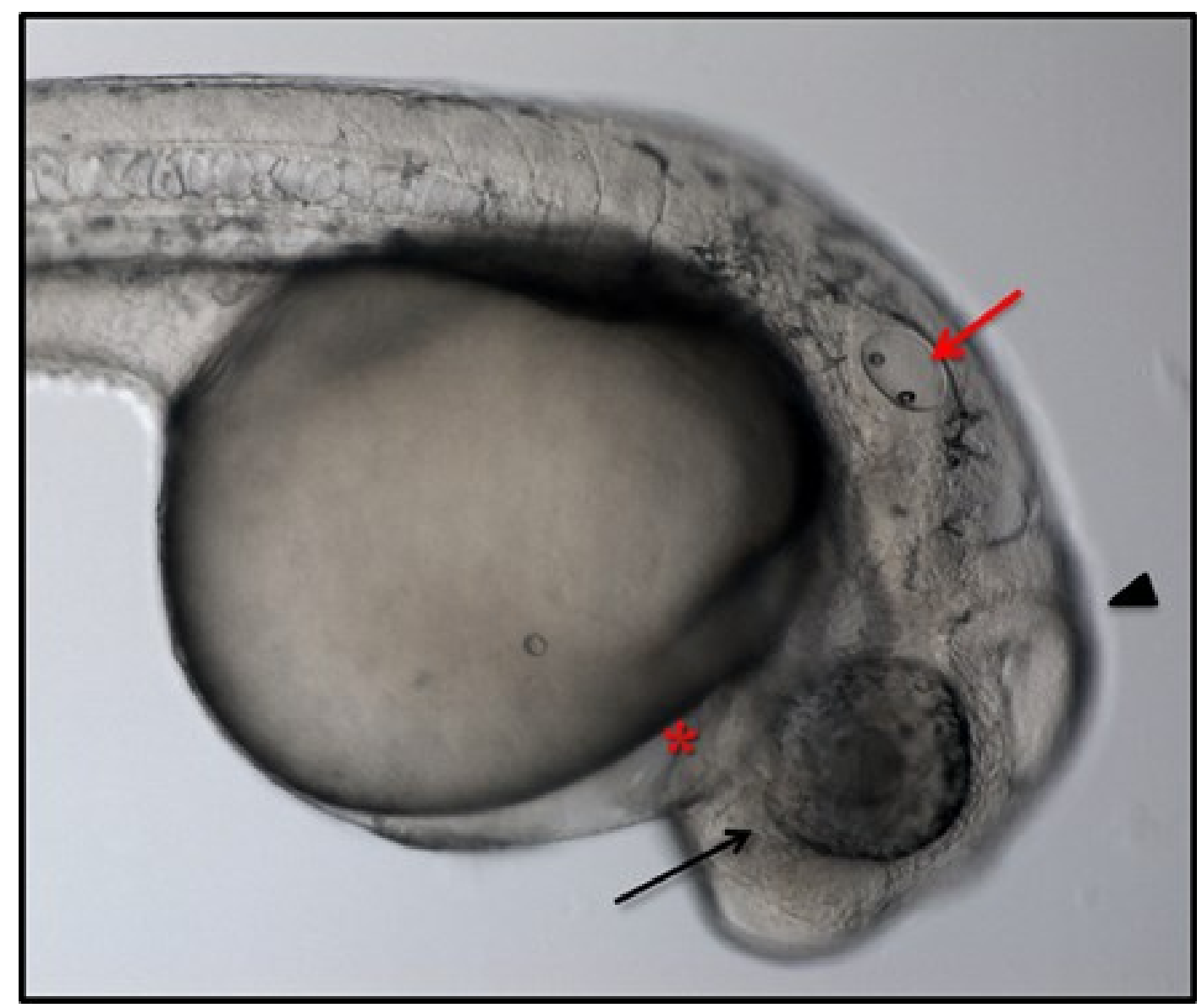

Figure 1-2. Zebrafish are a suitable model organism for studying developmental biological processes

At 1 days post-fertilization (dpf), the zebrafish embryo already has a developing ear (red arrow), brain (black arrowhead), eye (black arrow) and beating heart (red asterisk). 


\section{Zebrafish as a Model to Study Blood-Brain Barrier Development}

Within the past decade, scientists have exploited the advantages of the zebrafish model system to study the BBB. Other groups as well as our own have demonstrated the zebrafish BBB is comparable to that in mammals. The zebrafish BBB possesses tight junctions anchored between $\mathrm{BBB}$ endothelium maintaining a physical barrier and chemical transport systems mediating active efflux and prohibiting xenobiotic entry ${ }^{61-66}$. The tight junction protein Claudin5 is the most highly expressed homolog of the Claudin family in zebrafish ${ }^{67}$. Knockdown of Claudin5a in zebrafish reduces ventricle lumen expansion but does not affect neuroepithelial tissue integrity ${ }^{68}$. The zebrafish genome duplication event resulted in Claudin5a and Claudin5b alleles. Each paralog's tissue specificity has not been fully resolved and this may explain why Claudin5a knockdown may have an effect on the blood-CSF barrier and not at the $\mathrm{BBB}^{67}$. Functional studies in zebrafish are starting to occur as Fischer et al (2013) recently demonstrated the influx of the substrate Rhodamine with chemical and genetic inhibition of zebrafish $a b c b 4$, what they deem as the zebrafish ortholog to MDR $1^{66}$. Further investigation of bioavailability of compounds at the BBB still needs to be established. Zebrafish possess multi-drug resistance proteins in the gut epithelium and can actively prohibit the absorption of doxorubicin, a common MDR1 substrate ${ }^{65}$. Because the gut lumen and BBB maintain a similar barrier phenotype, it is possible that Mdr1 expressed at the zebrafish BBB also excludes compounds similarly ${ }^{69}$. All of these properties confer a functional BBB as it has been established in mammalian models ${ }^{61}$.

Neural vascular biology correspondingly develops in fish as it does in mammals (Figure 1-3). Development of the zebrafish primordial hindbrain channels (PHBC) commences around $28 \mathrm{hpf}$ in the zebrafish brain similarly as the formation of the perineural vascular plexus around E8.5 in the mouse and E11 in the rat ${ }^{27,35,70}$. Growth factors, including vascular endothelial cell growth factor (VEGF) signaling, have been widely established as important and specific mitogens in vessel formation ${ }^{71}$. Heterozygous lethality of one VEGF allele demonstrates the importance of this signaling pathway in blood vessel development ${ }^{72}$. Various zebrafish VEGF mutants also demonstrate genetically distinct VEGF profiles for particular blood vessel types ${ }^{73}$.

After vasculogenesis, nascent blood vessels sprout into the developing brain parenchyma. This process of newly formed vessels budding off of pre-existing ones is called angiogenesis ${ }^{74}$. Our lab has demonstrated CNS angiogenesis occurs similarly at $30 \mathrm{hpf}$ from zebrafish PHBCs as it does from the mouse perineural vascular plexus at E9.5-10 ${ }^{35,36}$ (Taylor lab, data unpublished). In the zebrafish, the main arterial route and vessel branching becomes hearty by $2 \mathrm{dpf}^{70}$. In addition to CNS angiogenesis, markers of barriergenesis also differentiate similarly in the zebrafish. GLUT1 has been identified as one of the initial markers of cerebral angiogenesis in mammals appearing at E12 in rat and $\mathrm{E} 11$ in mouse BECs ${ }^{28,75}$. Zebrafish also specifically express Glut1 early in angiogenesis suggesting that brain endothelial cells differentiate during initial CNS angiogenesis $^{64}$ (Taylor lab, data unpublished). Recently, it has been established that zebrafish express brain pericytes ensheathing endothelium as early as $48 \mathrm{hpf}$ and brain development ${ }^{76,77}$. The difference in timing of pericyte versus astrocyte 


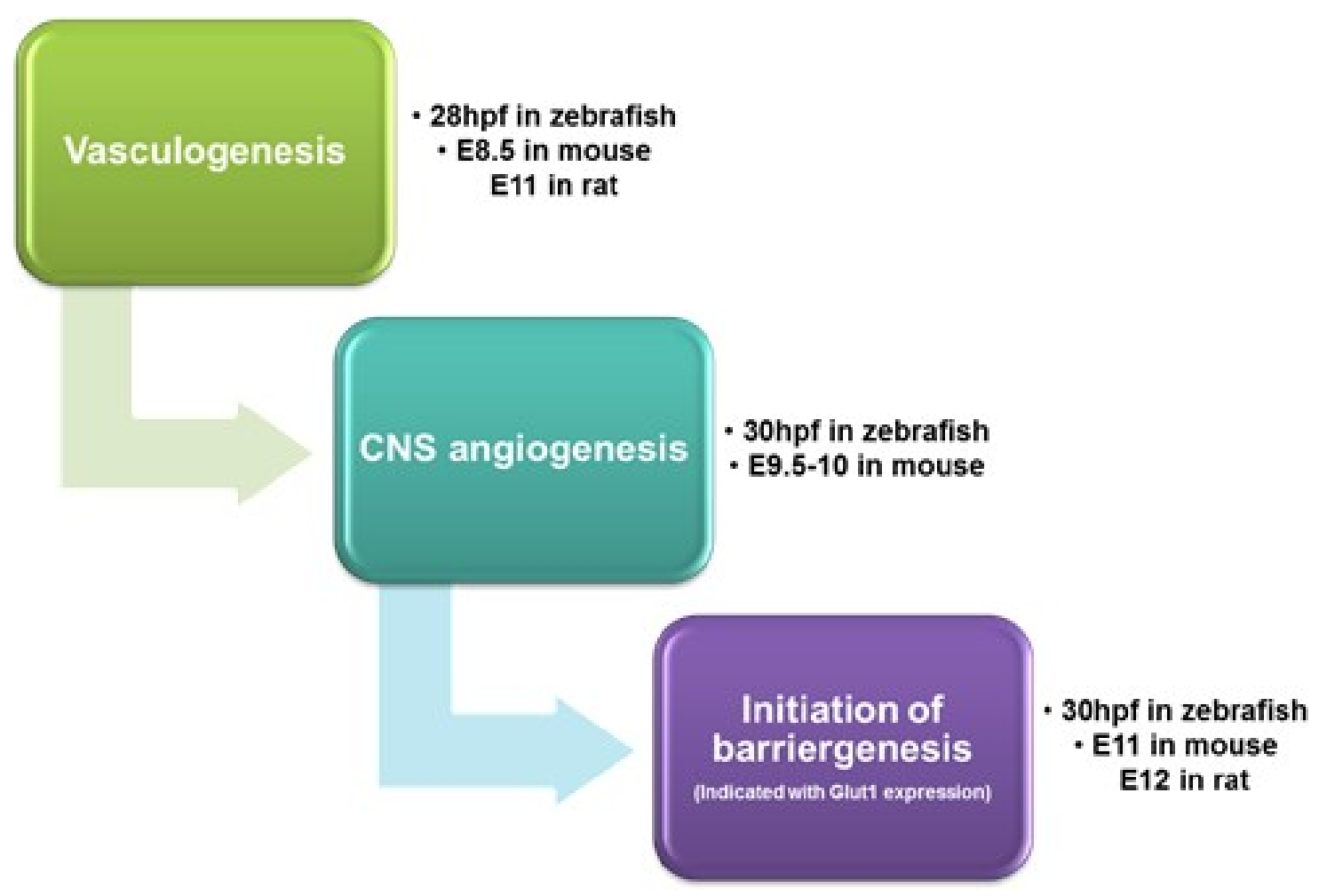

Figure 1-3. Comparative timeline of events between zebrafish and mammals during blood-brain barrier development

Zebrafish vasculogenesis, CNS angiogenesis, and barriergenesis occurs similarly as it does in rodents. The literature suggests that these two processes occur simultaneously, but a model has yet to show this in real time. The advantages of the zebrafish model system could be used to more carefully dissect these processes. 
development in the zebrafish brain may be an artifact of evolution, lack of transgenic lines to look at pericyte development in real time, as well as a limitation on established zebrafish astrocyte markers. For example, grey matter "protoplasmic" astrocytes surrounding CNS blood vessels express distinctive markers from the fibrous astrocytes that are typically labeled with the commonly used glial fibrillary associated protein marker $^{78}$.

Zebrafish provide an excellent model system for identifying canonical signaling pathways as well as novel signals important to development. Interestingly enough, expression profiles in mouse brain vasculature can be exploited in the zebrafish BBB. Tam et. al (2012) demonstrated the death receptors DR6 and TROY are important for both mouse and zebrafish barriergenesis ${ }^{64}$. These death receptors were also shown to be regulated by Wnt signaling, suggesting conservation in BBB signaling pathways between mammals and zebrafish ${ }^{64}$. Notch signaling has also been identified as an important regulator of barriergenesis in the mouse and has now been validated in the zebrafish ${ }^{76,79}$. The field of brain barrier biology could therefore benefit from further exploiting the zebrafish model system to uncover novel signaling pathways in high-throughput genetic and chemical screens.

\section{Hypothesis and Scope of This Dissertation}

The ultimate goal of this project was to use the zebrafish model for unbiased genetic and small molecule screens. These in vivo strategies could uncover novel molecular constituents that are important in barriergenesis. Therefore, we hypothesized that zebrafish can be used to genetically dissect the molecular mechanisms important for the development, function and maintenance of the BBB. To test our hypothesis, we completed the following specific aims:

1. Produce a BBB reporter line to characterize the in vivo development of the BBB.

We hypothesize that the creation of transgenic reporter lines using genes important for barrier function will allow us to study the development of the BBB. We first plan to create and then characterize the BBB reporter line, to survey the in vivo development of the BBB. By performing proof-of-principle experiments on our transgenic model, we can demonstrate that this reporter is a powerful tool for studying BBB development in a live animal and therefore useful for high-throughput screens.

2. Perform a genetic dissection of the BBB to uncover novel molecules that affect BBB properties.

A forward genetic and chemical screen exploiting our BBB reporter line could uncover mutants and compounds that cause defects in CNS angiogenesis and reporter expression. We will perform a small-scale F3 genetic screen after ENU treatment of adult BBB transgenics. Simultaneously we will test different compound libraries in wild- 
type BBB transgenics to look for alteration in BBB structure and reporter expression. These approaches are not only unbiased, but present the possibility of uncovering unfamiliar mechanisms important in barriergenesis. The execution of this screen will not only advance our understanding of BBB development but potentially reveal new genes from cloning mutants and "hits" that could be targeted for CNS drug therapy. 


\section{CHAPTER 2. GENERATION OF A BLOOD-BRAIN BARRIER REPORTER LINE}

\section{Introduction}

\section{Transgenics Used to Study the Blood-Brain Barrier}

Transgenic animals are modified to express non-native genetic sequences and have been studied to investigate developmental processes, tissues, and disease $\mathrm{s}^{47,80,81}$. These models are typically constructed by placing a specific promoter sequence upstream of a gene that will express some bioluminescent or fluorescent protein. However, there are currently no published models to study the BBB whereby a promoter localizes a reporter gene only to BECs. Instead, many groups utilize endothelial cell promoters to study BEC development even though all blood vessels are labeled. For example, the Tie2-GFP line is commonly used to study BBB development in the mouse ${ }^{35,36,40}$. The Tie2 promoter, an endothelial specific receptor tyrosine kinase, was used to create and understand vascular endothelial cell growth and function ${ }^{82}$. Studies for zebrafish BBB development have also utilized a similar transgenic line, $\operatorname{Tg}(f l i 1 a: E G F P)^{y 1}$ (herein $\operatorname{Tg}(f l i 1 a: E G F P)$ ) which labels blood vessels throughout the whole organism with the friend leukemia virus integration la (fli la) promoter driving expression of the enhanced green fluorescent protein (EGFP) reporter gene ${ }^{61,80,83}$. These transgenics are sufficient to study vessel development, but their promoter sequences are not specific to brain endothelium. More recently, the $\operatorname{Tg}(l-f a b p: D B P-E G F P)$ zebrafish reporter was generated to study the development and maintenance of brain barriers based on the expression and circulation of a $78 \mathrm{kDa}$ (kilo Dalton) Vitamin-D binding protein fused to EGFP ${ }^{62}$. The $T g(l-f a b p: D B P-E G F P)$ line was useful for studying the functional development of the $\mathrm{BBB}$ however, this transgenic does not report on the barrier signals that are essential for maintaining BBB function. If a BBB reporter line existed, studies could be performed to target pathways that affect molecular properties that constitute BECs versus signals that pertain to all endothelium. Therefore, the field of BBB biology could benefit from the construction of a zebrafish BBB reporter line.

\section{Tol2 Transgenesis}

To generate tissue specific reporter lines, zebrafish labs commonly use the Tol2Kit. ${ }^{84}$ This system is ideal for transgenesis as compared to injection of regular plasmid Deoxyribonucleic Acid (DNA) which gives lower germline transmission rates. ${ }^{84}$ Construct integration with $T o l 2$ can also be visualized by fluorescent reporter gene expression. It has been established that the Medaka fish, Oryzias latipes, possesses a transposable system deemed Tol2-tyr which can move in a cut and paste manner. ${ }^{85}$ While zebrafish do not contain Tol2 elements, co-injection into single cell zebrafish embryos of a Tol2 element with Tol2 transposase transcribed in vitro results in germ-line integration of the Tol2 element. ${ }^{86}$ The Tol2Kit uses site-specific recombination-based 
cloning that can produce a final clone containing a promoter sequence upstream of a reporter gene. ${ }^{84}$ Based on previous reports of the Tol2 system, a transgenesis rate of $50 \%$ is expected. ${ }^{87}$ Furthermore, the Tol2Kit has successfully produced transgenics such as those using the bactin 2 and $h s p 70$ promoters to drive GFP expression. ${ }^{84}$

\section{Materials and Methods}

\section{Zebrafish Lines}

The $\operatorname{Tg}(f l i l a: E G F P)$ strain was acquired from the Zebrafish International Resource Center (ZIRC). To assess glut lb expression patterns, we used two other transgenics, $c p: E G F P$, an enhancer trap line generated in our lab that expresses EGFP in the choroid plexus and tac:EGFP, a transgenic line that expresses EGFP in photoreceptor cells and the pineal gland. Embryos and larvae were maintained at $28.5^{\circ} \mathrm{C}$ in egg water (0.03\% Instant Ocean). $0.003 \% \mathrm{~N}$-Phenylthiourea (PTU) was used to inhibit melanin production for imaging. Dr. Taylor injected $2 \mathrm{nl}$ of $1 \mathrm{mg} / \mathrm{ml}$ bovine serum Albumin, Alexa Fluor 647 Conjugate (Invitrogen) into the common cardinal vein and fish were imaged by confocal microscopy

\section{Tol2 Transgenesis and Construct Microinjection}

The generation and injection of the BBB construct was performed in a group effort by multiple lab members. To generate the construct for $\operatorname{Tg}(\text { glut } 1 b: m C h e r r y)^{s j l}$, we used Gateway compatible vectors of the Tol2kit. A $2.9 \mathrm{~kb}$ fragment of the zebrafish glut $1 b$ promoter (accession \#: NM_001039808) was amplified from genomic DNA using a forward primer of 5'-TATTctcgagGGGGCTGATAACATTGACCT (with a Xho I restriction site added, lower case) and a reverse primer of 5'TCCAggatccCAAAAATTGTTCTTTAAAAAAAAC (with a Bam HI site added, lower case) and subcloned into the TOPO TA cloning vector (Invitrogen). The glut $1 b$ promoter was released from the cloning vector by XhoI and BamHI double digestion and inserted into $p 5 E-M C S$ (Tol2kit). This 5' entry clone was combined with middle entry clone pME-mCherry, the 3' entry clone $p 3 E$-polyA, and the pDestTol2pA2 destination vector to create the pDest-glut 1b:mCherry construct using the LR Clonase II Plus Enzyme mix (Invitrogen). Approximately $30 \mathrm{pg}$ of plasmid DNA and $30 \mathrm{pg}$ of in vitro transcribed Tol2 transposase messenger ribonucleic acid (mRNA) were co-injected into single-cell $T g$ (flila:EGFP) embryos $(\mathrm{n}=45)$. All microinjections were performed using a PV820 Pneumatic PicoPump (WPI). The embryos were raised to adulthood and screened for germline transmission by observing mCherry expression in BECs. The $T g$ (glutlb: $m$ Cherry $)^{\text {sjl }}$ line was bred to homozygosity and determined to contain a single insertion site. 


\section{Live Imaging Confocal Microscopy}

For imaging, fish (1-6 dpf) were anesthetized in $0.02 \%$ Tricaine and immobilized in $1.2 \%$ low melting point agarose (Invitrogen) in glass bottom culture dishes (MatTek). Confocal microscopy was performed on a Nikon TE2000E2 microscope equipped with a Nikon C1Si confocal using 488nm, 561 nm and 638nm DPSS lasers for excitation.

Images were acquired with a Nikon 20X 0.75 NA Plan Apo DIC objective and processed using Nikon NIS Elements software. Confocal z stacks were acquired with a 3 micron step size; for time-lapse imaging, a $\mathrm{z}$ stack was acquired every 30 minutes for 30 hours. All images are 2D projections of 3D confocal z-stacks, generated using either a MIP or EDF algorithm. Time-lapse movies were further processed by applying a Gaussian blur to the image stack before creating the $2 \mathrm{D}$ projection in order to remove "noise" from the collection of pixels.

\section{Adult Dissection}

$\operatorname{Tg}$ (flila:EGFP) and $\operatorname{Tg}$ (glut 1b:mCherry) adults were deeply anesthetized with $0.04 \%$ tricaine made in system water and humanely sacrificed for dissection. Dissections were performed on a petri dish containing a Kim wipe soaked in $0.04 \%$ tricaine. Tissues were dissected using a razor blade and fine forceps. Organ images were acquired on a Nikon SMZ 1500 stereoscope with a PRIOR Scientific Lumen 200 PRO Fluorescence Illumination System.

\section{Results and Discussion}

\section{Selecting a BBB-Promoter, Glucose Transporter 1}

BECs possess chemical and physical barriers to maintain brain homeostasis. The chemical barrier is mediated by transport systems, which may be metabolic, xenobiotic or solute specific. Carrier-mediated efflux pumps are crucial for delivering nutrients to the brain that would not otherwise be accessible. Glut1, one of these main transporters, is responsible for delivering glucose to the brain, as it requires $20 \%$ of whole body glucose $^{26}$. Because of this physiological need, GLUT1 is highly expressed the $\mathrm{BBB}^{7,39,88}$ GLUT1 is also one of the earliest markers at the BBB, and several recent studies have used GLUT1 expression as a functional indicator of BBB formation ${ }^{36,41}$. It has also been reported that BBB tightness plays a pivotal role in the pattern of GLUT1 expression during brain differentiation, demonstrating that GLUT1 serves as an indicator of BBB function as well as development ${ }^{28,75}$. Furthermore, it has been demonstrated that Glut1 localizes to BECs in zebrafish ${ }^{64}$. Therefore, based on its significance in BBB development and expression, we selected the zebrafish Glut1 homolog as a molecular tool for creating our BBB transgenic line. 
To visualize in vivo development of the BBB, we created a novel transgenic zebrafish reporter line using the zebrafish glut $1 b$ promoter. While zebrafish have three glut1 paralogs, we focused our studies on glut1b (NM_001039808), due to its predominant expression of mRNA in the brain ${ }^{63}$. We chose mCherry for our BBB reporter because it matures more rapidly than other fluorescent proteins ( $t_{0.5}=15$ minutes) allowing for accurate observation of temporal expression ${ }^{89}$. Injections were performed in the $T g(f l i 1 a: E G F P)$ line so we could look for an overlap with glut $1 b$ expression in EGFP positive BECs (Figure 2-1). Generating the BBB line with a transgenic labeling all endothelium would also allow us to study processes that affect BECs separately from the periphery. We found mCherry transiently expressed in the brain vasculature of $\sim 90 \%$ of injected embryos (Figure 2-2).

\section{Visualization of BBB in vivo Development}

We identified several stable transgenic lines by out-crossing transient expressers to $T g(f l i l a: E G F P)$ and designated one as $T g$ (glut $1 b: m C h e r r y)^{\text {sjl }}$ (herein $\operatorname{Tg}($ glut 1 b:mCherry $))$. To characterize the expression pattern of mCherry, we used confocal laser scanning microscopy on live glut 1b:mCherry; fli la:EGFP double transgenics. At $6 \mathrm{dpf}$, mCherry was expressed specifically in the brain vasculature (Figure 2-3C) and in the myocardium (data not shown), but not in the peripheral vasculature (Figure 2-3F). This expression pattern was maintained in adulthood as well as in the reproductive tissues of the adult (Figure 2-4). GLUT1 is expressed in ovarian tissue among different species, yet there are still species specific differences in tissue expression patterns ${ }^{90}$. When the cavities of the adults were opened, it appeared as if viscera like the kidney (data not shown) may also be glut $1 b$ positive which has also been reported in other species as glucose transport is involved in hyperglycemia and diabetes $\operatorname{progression}^{91}$.

Brain vessels are very specialized so that they do not easily allow the free exchange of molecules into the brain. One of these specializations includes the lack of fenestrations, openings in the vessel wall, a non-barrier property which is commonly seen in circumventricular organs ${ }^{92}$. Fenestrations are not only present in these organs but have also been demonstrated as marker for BBB disruption ${ }^{93}$. To demonstrate that mCherry expression was specific to endothelium that maintain BBB properties, we looked at $m C h e r r y$ in circumventricular organs in other transgenic lines. We crossed $T g$ (glut1b:mCherry) to $c p: E G F P$, an enhancer trap line that expresses EGFP in the choroid plexus and tac:EGFP, a transgenic line that expresses EGFP in the pineal gland. We found that mCherry was not expressed in the choroid plexus or pineal gland vasculature (Figure 2-3J and $\mathbf{N}$ ), indicating that glut 1b:mCherry in the zebrafish faithfully recapitulates mammalian GLUT1 expression at the BBB.

Taking advantage of our in vivo model, we wanted to address a fundamental argument about BBB development. Ongoing hypotheses suggest that BBB formation is either 1) a two-step process where CNS angiogenesis occurs and is followed by barriergenesis, or that 2) both CNS angiogenesis and barriergenesis occur at the same 
DNA Construct

\section{$B B B$ promoter mCherry}

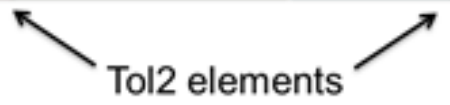

\section{To/2 transposase mRNA AAAAAAAAAA}

Micro-injection

in single cell

$\mathrm{Tg}($ fli1a:EGFP)

embyros

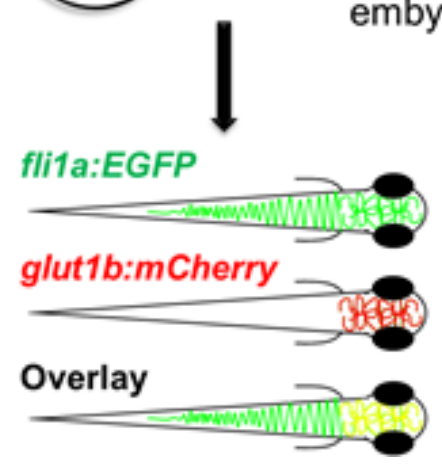

Figure 2-1. Blood-brain barrier construct injection into $T g(f l i 1 a: E G F P)^{y 1}$ embryos

A BBB reporter construct was engineered with the Gateway Tol2kit cloning system. This DNA element contained a $2.9 \mathrm{~kb}$ fragment of the zebrafish glut $1 \mathrm{~b}$ promoter upstream of the mCherry reporter gene. The DNA construct contained Tol2 sites flanking the transgene, so that upon co-injection with Tol2 transposase mRNA into single cell $\operatorname{Tg}$ (flila:EGFP) embryos, the construct would be inserted into the genome of the developing zebrafish. We hypothesized that if the glut $1 b$ promoter was specific to BECs, we would see a yellow overlay with the red signal from glut $1 b$ and the green signal from flila. 

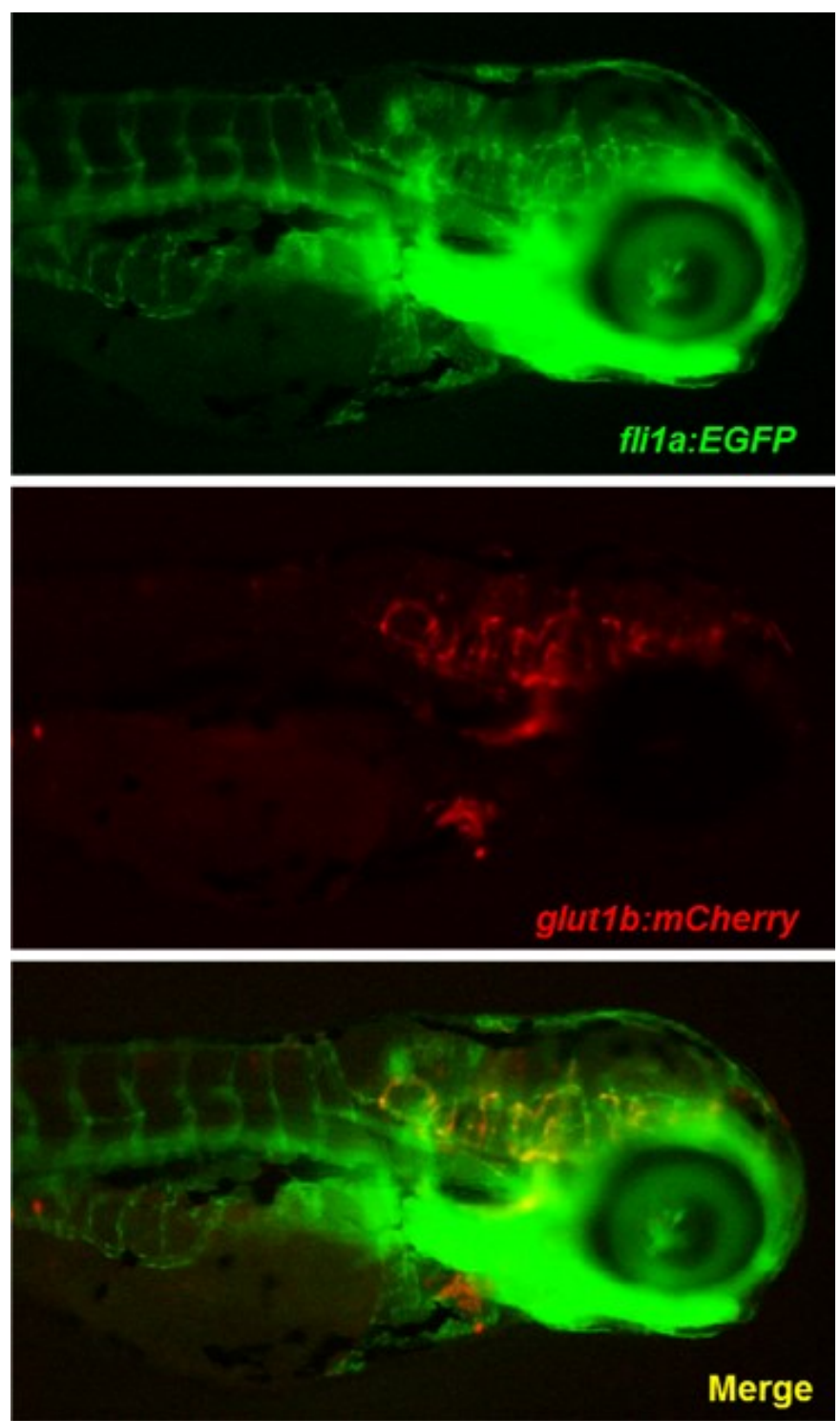

Figure 2-2. Transient expression of glutlb:m Cherry in flila:EGFP brain endothelium

After co-injection of the Tol2 transposase mRNA and the glut $1 b$ construct, glut 1b:mCherry localized to flila:EGFP BECs as seen here with a representative $3 \mathrm{dpf}$ transgenic animal (anterior, right; dorsal, top). Transient expression also reveals that the promoter does not localize mCherry to peripheral blood vessels. 

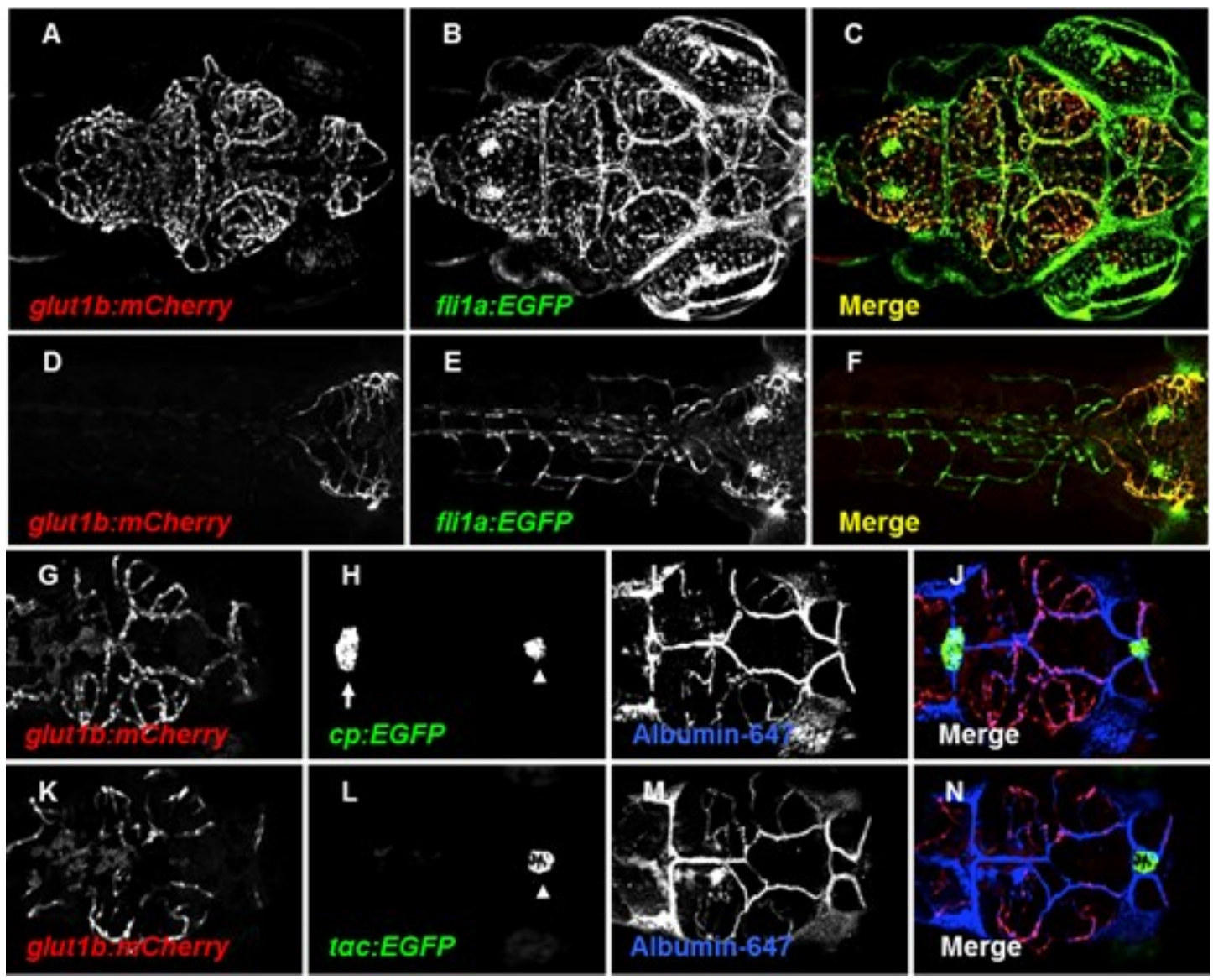

Figure 2-3. glut1b is expressed in brain endothelial cells and not in circumventricular organs

Upon germline transmission of the glut 1b:mCherry transgene, mCherry was still restricted to the brain vessels of a $6 \mathrm{dpf}$ animal $(\mathbf{C})$ and not the peripheral vasculature $(\mathbf{F})$. We crossed $T g$ (glut 1b:mCherry) to two other transgenics that expressed EGFP in tissues that have fenestrated endothelium, the choroid plexus (H arrows and arrowhead) and pineal gland (L arrowhead ). These lines were also injected with an Albumin tracer labeled in the far red channel to demonstrate that these BECs were functional. As seen from the merge panels, glut $1 b$ is not expressed in capillaries of the choroid plexus $(\mathbf{J})$ or pineal gland $(\mathbf{N})$. 

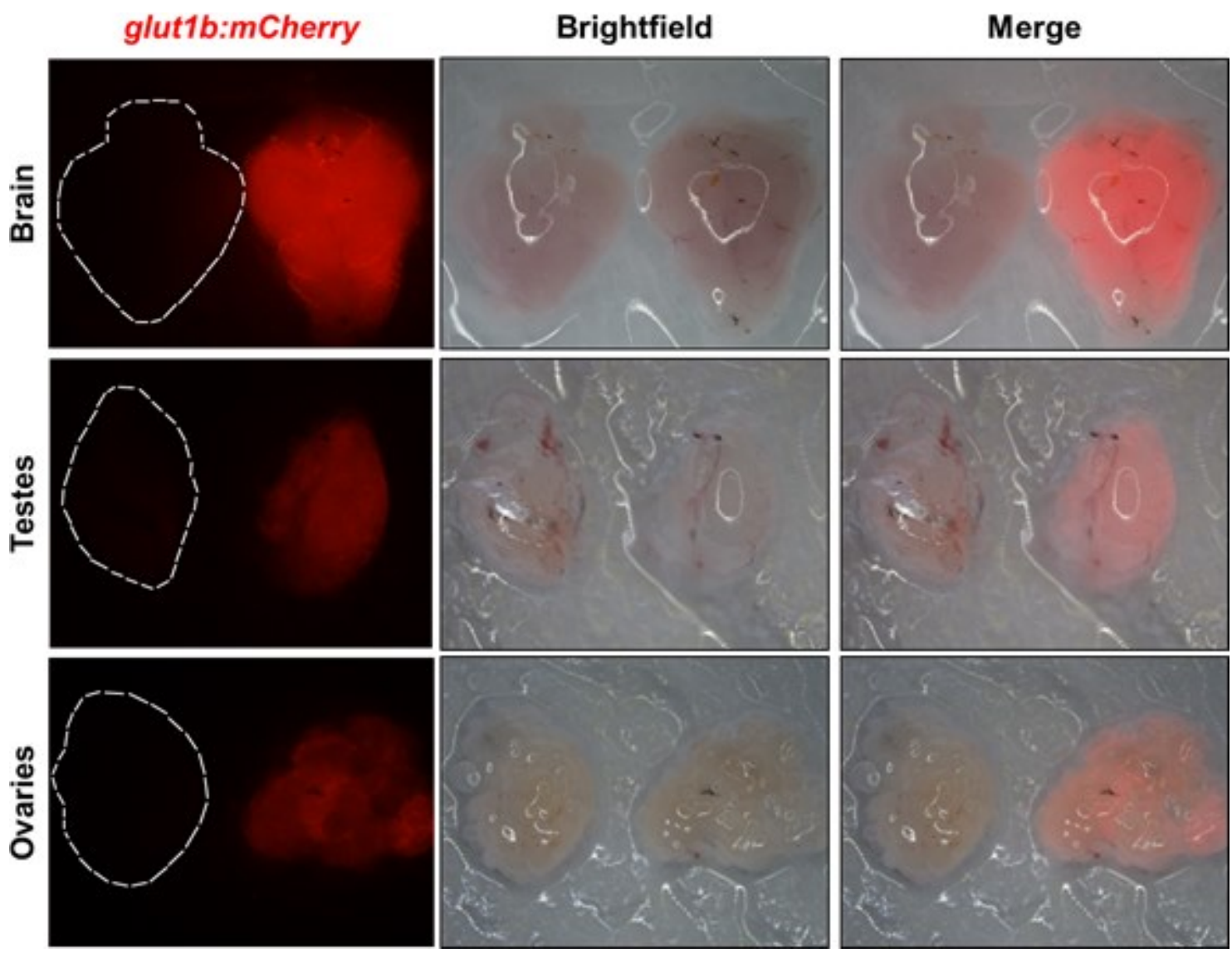

Figure 2-4. glut1b:mCherry is maintained in adult brain endothelial cells as well as reproductive tissues

$T g$ (flila:EGFP) (left tissues) and $T g$ (glut1b:mCherry) (right tissues) adults were dissected to look at glut $1 b$ expression in adulthood. glut $1 b$ still localized to BECs in adulthood and was also seen in reproductive tissues like the ovaries and testes. 
time. The "two-step" hypothesis is largely based upon observations that immature CNS blood vessels are leaky to injected tracers such as Evans Blue. However, the conclusions from these studies have been challenged due to the methodology used ${ }^{1}$. While growing evidence indicates that barriergenesis and CNS angiogenesis may be linked, the inability to visualize these processes in real time limits the observation of the earliest steps of CNS vascular development ${ }^{36}$. To address this issue, we performed time-lapse confocal microscopy to examine the temporal induction of barrier properties. In zebrafish, CNS angiogenesis begins in the hindbrain at approximately $30 \mathrm{hpf}$ when endothelial tip cells originating from the PHBCs migrate into the brain parenchyma ${ }^{70}$. These vessels eventually form the central arteries (CtAs) that interconnect the PHBCs with the basilar artery. Using live glut 1b:mCherry; flila:EGFP double transgenics, we imaged angiogenesis and barriergenesis from 30 to $60 \mathrm{hpf}$. We observed that endothelial cells express mCherry immediately upon entering the brain (Figure 2-5), indicating direct evidence for the hypothesis that barriergenesis and angiogenesis occur simultaneously.

\section{Conclusion}

A comprehensive understanding of the development and maintenance of the BBB has been hampered by difficulties in observing the $\mathrm{BBB}$ in vivo. To do this, we generated a transgenic zebrafish line, $\operatorname{Tg}$ (glut lb:mCherry) $)^{\text {sil }}$, to serve as an in vivo reporter of the BBB. These animals were used for imaging live BBB formation, a process that has not been able to be performed until now. We show that our transgenic line drives expression just like the mammalian GLUT1 ortholog, specifically in BECs and not in the vasculature of peripheral tissues or circumventricular organs with fenestrated capillaries. This expression pattern is maintained until adulthood as well. Furthermore, we show evidence for the first time that the processes of barriergenesis and angiogenesis happen concurrently in a live animal. 


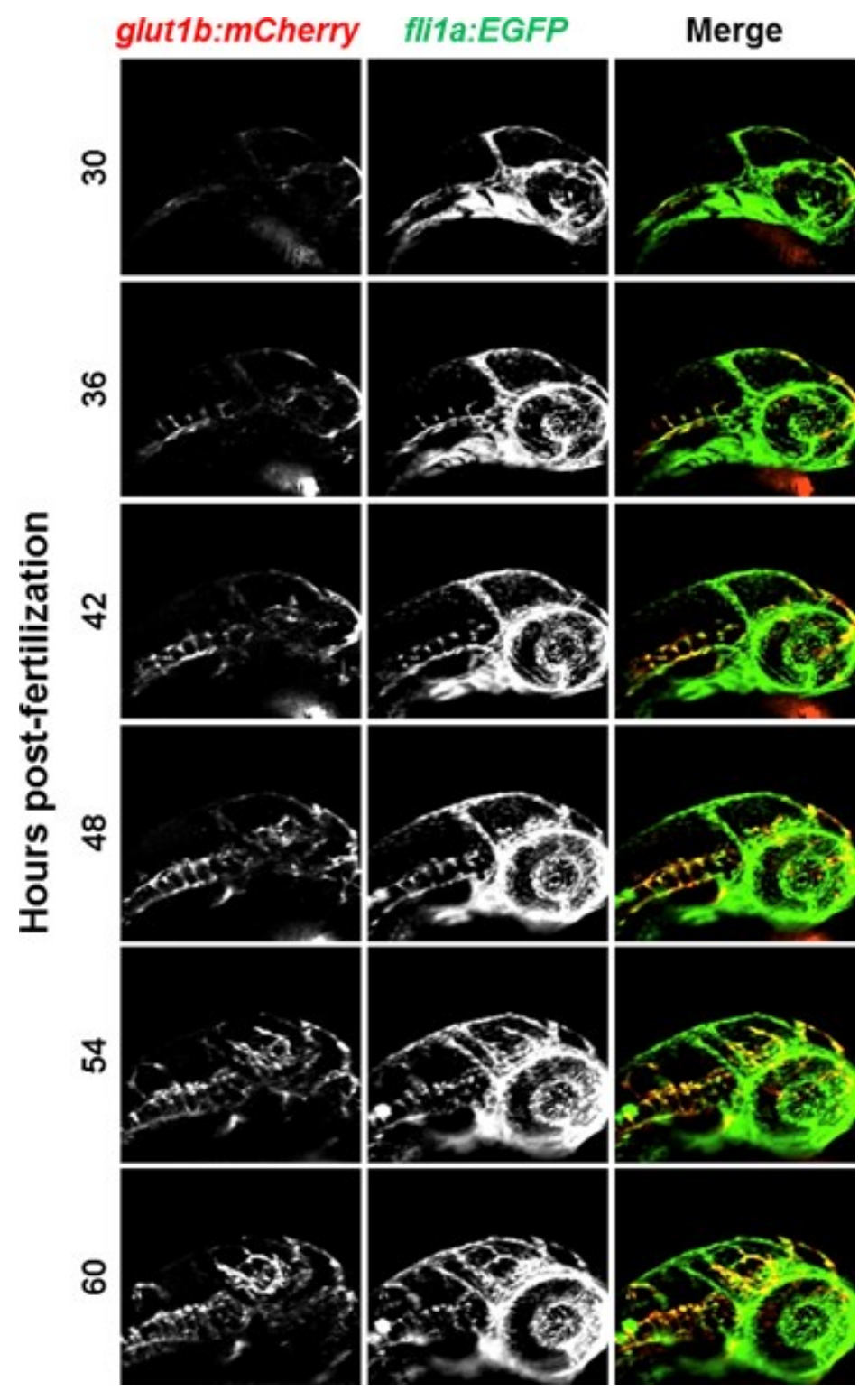

Figure 2-5. Angiogenesis and barriergenesis occur simultaneously Stills were taken from a live time-lapse movie of glut $1 b: m$ Cherry development. As soon as flila:EGFP positive tip cells migrated into the zebrafish brain parenchyma at $30 \mathrm{hpf}$, the endothelium turned positive for glut1b:mCherry expression. This expression is visualized over the 30 hour movie. 


\section{CHAPTER 3. CHARACTERIZATION OF THE BLOOD-BRAIN BARRIER REPORTER LINE*}

\section{Introduction}

\section{Characteristic Properties of the BBB}

The BBB is well-known for restricting xenobiotic entry into the CNS while still providing the proper nutrition for brain tissue. Since the start of the $20^{\text {th }}$ century, the $\mathrm{BBB}$ has been known to maintain a functional partition between the circulatory system and the $\mathrm{CNS}^{17-19}$. It was not until quite some time later, that seminal transplant studies elucidated the importance of the brain milieu for a functional barrier ${ }^{32}$. Electron microscopy later revealed that tight junction proteins in between brain endothelium regulate the passive transport of substances into the brain tissue ${ }^{21}$. Multi-drug resistant proteins found up-regulated in cancerous tissues were also found localized to BECs as a mechanism to explain poor drug penetration in the brain ${ }^{8}$. Localization of transport systems in the BBB was also congruent with the highly metabolic nature of the brain ${ }^{28,75}$. Together, these specific proteins located along the brain endothelium are what give the $\mathrm{BBB}$ its special properties and function.

\section{Methods for Testing the Function and Structure of the BBB}

Functional Protein Markers. The features responsible for creating the physical and chemical barriers in the CNS have been widely established and are used as traditional markers of BBB integrity. In addition to studying the normal protein expression of each of these markers, multiple knock-out animals have been generated to assess their biological contributions to CNS barriers $22,24,94,95$. Tight junction proteins anchored between BECs are responsible for regulating the free exchange of factors that circulate between the blood and the CNS. Tight junctions compose an intricate network to tether brain endothelium and are vulnerable to destruction in various disease states ${ }^{2}$. In fact, each Claudin family member regulates the access of different sized molecules ${ }^{2,22}$. Claudins are expressed early in zebrafish development and lack of functional Claudin affects the blood-CSF barrier ${ }^{62,67}$. The ABC transporter family proteins, BCRP and MDR1 are two of the most widely studied features in the chemical barrier that maintain drug distribution throughout the $\mathrm{BBB}^{7}$. While these proteins have been thoroughly established in the mammalian $\mathrm{BBB}$, recent zebrafish work has demonstrated the presence of multi-drug resistance proteins in fish as well ${ }^{65,66,96}$. Studies have also used the

*Modified with permission. Umans, R. A. \& Taylor, M. R. Zebrafish as a model to study drug transporters at the blood-brain barrier. Clin Pharmacol Ther 92, 567-570, doi:10.1038/clpt.2012.168 (2012). 
expression of carrier-mediated transporters like GLUT1 to demonstrate functional integrity of the $\mathrm{BBB}^{27,75}$. In fact, GLUT1 haploinsufficiency results in high brain dysfunction and elucidates the importance of this transport system in the $\mathrm{CNS}^{2}$.

Tracer Injections. Erhlich's tracer injections between brain and the CSF were what initially identified a barrier between the blood and $\mathrm{CNS}^{17,18}$. Additional trypan blue injections performed by his graduate student in the early 20th century helped further establish the functional compartment between the blood and $\mathrm{CSF}^{19}$. Injections with trypan and Evans blue are not antiquated and still typically performed to assess the development of the BBB and its function in disease states ${ }^{27,97}$. However, caution should be taken when assessing these results as large injection volumes may cause staining of the brain in instances smaller volumes would not ${ }^{1}$. With the advancement in imaging techniques, fluorescence microscopy is also commonly used to look at the integrity of the BBB. Functional studies have utilized circulating proteins or tracers conjugated to fluorescent dextrans to visualize BBB function which is ideal in the optically transparent zebrafish system ${ }^{35,40,61}$.

\section{Materials and Methods}

\section{Immunohistochemistry (IHC)}

These experiments were performed in conjunction with the other graduate research assistant, Dr. Hannah Henson. For tissue sectioning, embryos were fixed in 4\% paraformaldehyde overnight at $4^{\circ} \mathrm{C}$, washed in 1x Phosphate Buffered Saline (PBS), and stored in $30 \%$ sucrose at $4^{\circ} \mathrm{C}$. Embryos were embedded in OCT and stored at $-20{ }^{\circ} \mathrm{C}$. Tissue sectioning was performed using a Leica CM 1950 cryostat. Transverse sections were done on $30 \mathrm{hpf}$ and $36 \mathrm{hpf}$ embryos and coronal sections were done on $48 \mathrm{hpf}-6 \mathrm{dpf}$ embryos.

For Glut1 and Mdr1 immunohistochemistry, primary antibodies included rabbit anti-Glut1 (1:200; Novus Biologicals) mouse anti-GFP (1:500; Invitrogen), mouse antiMdr1 (1:20, Calbiochem), and rabbit anti-GFP (1:500, Invitrogen). Secondary antibodies included goat anti-rabbit 555 (1:400; Invitrogen), goat anti-mouse 488 (1:1000; Invitrogen), goat anti-mouse 555 (1:200, Invitrogen), and goat anti-rabbit 488 (1:200, Invitrogen). Antibody dilutions were prepared in $1 \mathrm{xPBS} /$ Triton $0.03 \%$ (PBST). Sections were washed in 1x PBS for 5 minutes and in PBST 3 times for 5 minutes. Blocking was done in 5\% goat serum and incubated for 3 hours at room temperature. Primary antibodies were added overnight at $4^{\circ} \mathrm{C}$. Secondary antibodies were added for 2 hours at room temperature. Washes in PBST were performed after primary and secondary antibody incubations. Sections were DAPI stained, rinsed briefly in 1x PBS, and mounted with Fluoromount (Electron Microscopy Sciences) before adding the coverslip. All images were taken on a Nikon AZ100 microscope using NIS-Elements AR 3.2 software. 


\section{Live Imaging Confocal Microscopy}

For imaging, fish (1-6 dpf) were anesthetized in $0.02 \%$ Tricaine and immobilized in $1.2 \%$ low melting point agarose (Invitrogen) in glass bottom culture dishes (MatTek). Confocal microscopy was performed on a Nikon TE2000E2 microscope equipped with a Nikon C1Si confocal using 488nm, 561 nm and 638nm DPSS lasers for excitation.

Images were acquired with a Nikon 20X 0.75 NA Plan Apo DIC objective and processed using Nikon NIS Elements software. Confocal z stacks were acquired with a 3 micron step size. All images are 2D projections of 3D confocal z-stacks, generated using either a MIP or EDF algorithm. Time-lapse movies were further processed by Dr. Jennifer Peters by applying a Gaussian blur to the image stack before creating the 2D projection.

\section{Transgenic Wnt Inhibition by Induction of Axin-1}

Embryos from a $\operatorname{Tg}$ (glut 1b:mCherry;flila:EGFP) female crossed to a $T g(h s p 70 l$ 1:GFP-Axin1 $)^{z d 13}$ (herein $\operatorname{Tg}($ hsp70l-1:GFP-axin1)) male were transferred into a $1.5 \mathrm{ml}$ centrifuge tube with $0.5 \mathrm{ml}$ egg water/PTU and placed in a heating block set to $38^{\circ} \mathrm{C}$. Heat shock was performed at $24 \mathrm{hpf}$ and $48 \mathrm{hpf}$ for either $30 \mathrm{~min}$ or $60 \mathrm{~min}$ each. $\mathrm{Tg}$ (glut 1b:mCherry) embryos express mCherry in early development due to maternal deposits that obscure mCherry expression in BECs up to $3 \mathrm{dpf}$. Thus, the effects of heat shock-induced Axin 1 could not be easily visualized prior to $3 \mathrm{dpf}$. Therefore, a second heat shock at 48 hpf was necessary to maintain sufficient levels of Axin1 for imaging at $78 \mathrm{hpf}$.

\section{Small Molecule Treatment with VEGFR Inhibitors}

These experiments were performed by Dr. Michael Taylor. For drug treatments, AV-951 (Selleckchem), DMH1 (Tocris), DMH4 (Sigma), and Cyclopamine (Sigma) were prepared as stocks in $100 \%$ DMSO at $10 \mathrm{mM}$ and applied to $24 \mathrm{hpf}$ embryos at a final concentration of $1 \mu \mathrm{M}, 10 \mu \mathrm{M}, 10 \mu \mathrm{M}$, and $50 \mu \mathrm{M}$, , respectively.

\section{Troponin (tnnt2a) Morpholino}

We generated a tnnt $2 a$ morpholino (Gene Tools) using the previously published morpholino sequence ${ }^{98}$. A $2 \mathrm{mM}$ stock of morpholino was prepared upon receiving the lyophilized powder from Gene Tools. The stock was heated up at $55{ }^{\circ} \mathrm{C}$ for 5 minutes, allowed to cool down, and then prepared with $1 \%$ phenol red for injection.

Approximately $4 \mathrm{ng}$ of tnnt $2 a$ morpholino was injected into single-cell embryos from a Tg(glut1b:mCherry) $)^{\text {sjl }}$; $\operatorname{Tg}(\text { flila:EGFP })^{y 1}$ male X TL female cross, resulting in $>90 \%$ of embryos with no heartbeat. Embryos were imaged at $2 \mathrm{dpf}$. 


\begin{abstract}
Albumin Alexa Fluor 647 Injection
For tracer microinjection, Dr. Michael Taylor injected $2 \mathrm{nl}$ of $1 \mathrm{mg} / \mathrm{ml}$ bovine serum albumin, Alexa Fluor 647 Conjugate (Invitrogen) into the common cardinal vein and imaged by confocal microscopy. All microinjections were performed using a PV820 Pneumatic PicoPump (WPI). Injection volumes were calculated using a $0.01 \mathrm{~mm}$ Stage Micrometer (Fisher Scientific).
\end{abstract}

\title{
Results and Discussion
}

\section{Characterization of Zebrafish BBB Development}

After we achieved stable germline transmission with $\operatorname{Tg}($ glut $1 b: m C h e r r y)$, animals were mated to homozygosity. Our next goal was to perform proof-of-principle experiments to demonstrate the usefulness of our BBB reporter and that the BBB is comparable in fish and in mammals. To do so, we performed experiments to take advantage of the zebrafish's transparency, genetic tractability and ease in chemical treatment.

IHC with BBB Markers. We examined the zebrafish brain microvasculature for the presence of endothelial tight junctions and the expression of transporters localized to BECs. We found that zebrafish BECs express the tight junction protein Claudin 5 and the transporter Glut1 as early as 2 dpf, consistent with previous studies (Figure 3-1) ${ }^{62,64}$. Furthermore, we demonstrated for the first time that zebrafish BECs express the drug efflux transporter, Mdr1 throughout development (Figure 3-2 ${ }^{65}$. Together, these observations indicate that the zebrafish expresses BBB markers as do mammals.

Function of Newly Formed Vessels. We next examined the integrity of newly formed brain vessels since zebrafish are amenable to early embryonic visualization. We injected Albumin, Alexa Fluor 647 conjugate as a semi-endogenous permeability tracer into the cardinal vein of $T g(g l u t 1 b: m C h e r r y$; flila:EGFP) double transgenics prior to CNS angiogenesis and imaged from 30 to $60 \mathrm{hpf}$. We found that albumin was contained within the lumen of the vasculature as new vessels migrated into the brain parenchyma (Figure 3-3). These results show that physical barrier properties are present even at the earliest stages of brain vascular development, a hypothesis that is widely debated ${ }^{1}$. However, during this time course, some albumin appeared to enter the brain ventricles (Figure 3-3 asterisks). This ventricular "leakage" is likely due to the lack of a functional choroid plexus, which develops at $4 \mathrm{dpf}$ in zebrafish and is consistent with the lack of barrier properties in the choroid plexus vasculature at later developmental stages ${ }^{99,100}$. 
A

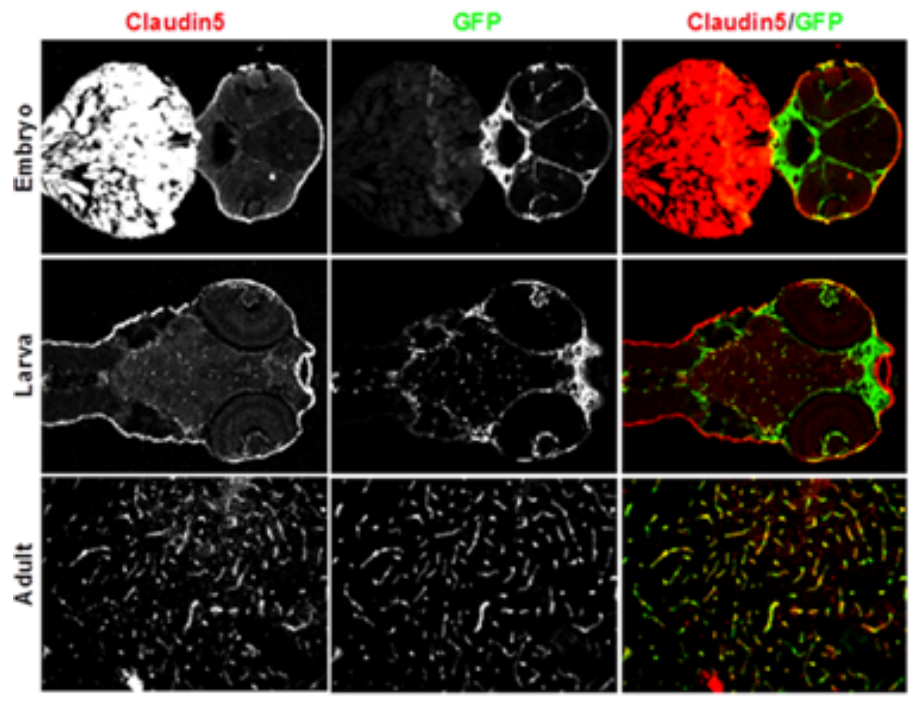

B

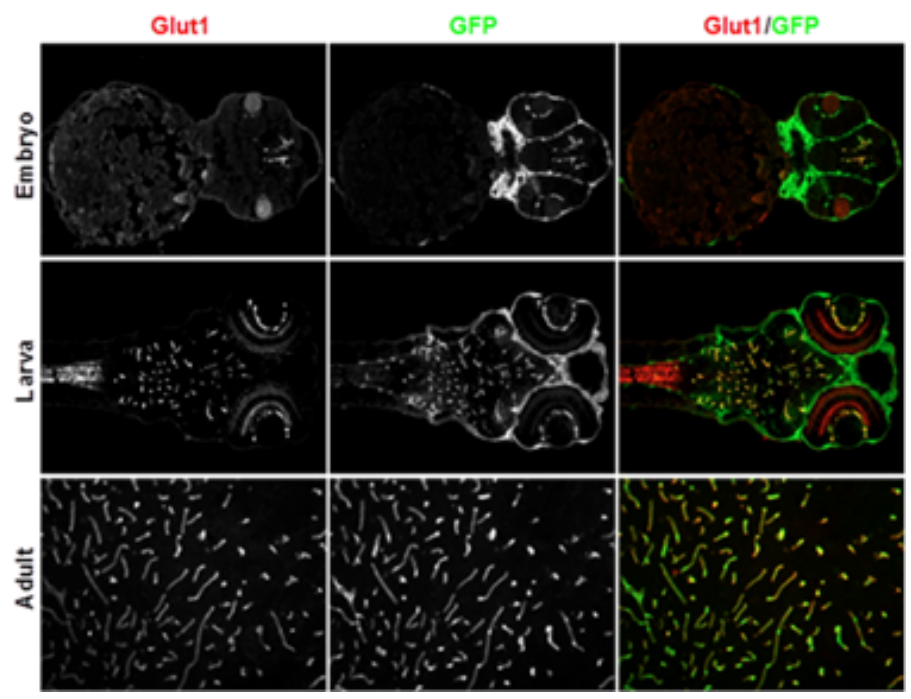

Figure 3-1. Zebrafish brain endothelial cells express Claudin5 and Glut1 during development

$\operatorname{Tg}$ (flila:EGFP) animals were sectioned to look at the co-localization of BBB markers to EGFP positive blood vessels. Just as the mammalian BBB, embryonic, larval, and adult zebrafish express tight junction proteins like Claudin5 (A) and efflux transporters like Glut1 (B) in BECs. 


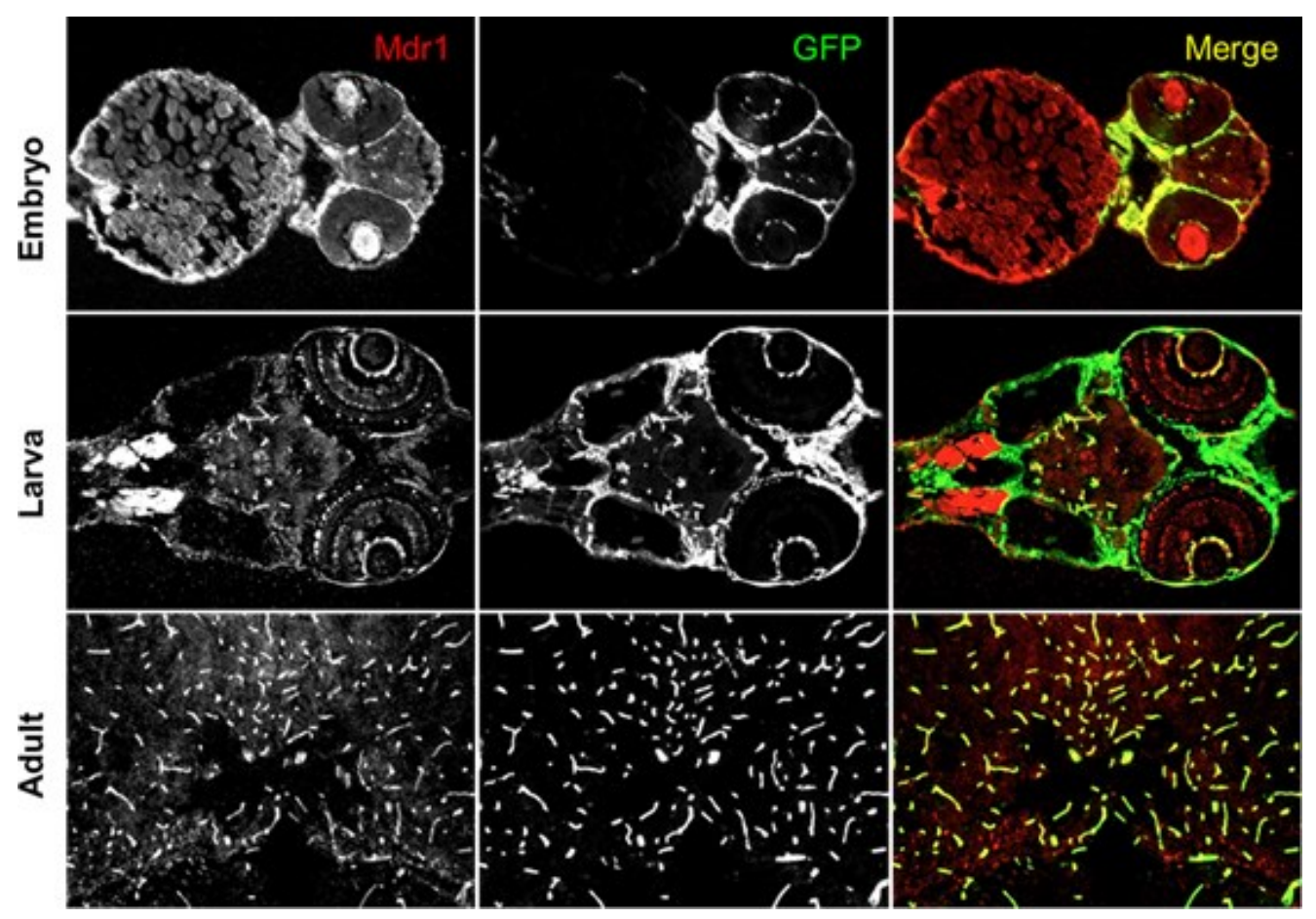

Figure 3-2. Zebrafish brain endothelial cells express Mdr1 during development $\mathrm{Tg}$ (flila:EGFP) animals were sectioned to look at the co-localization of Mdr1 to EGFP positive blood vessels. Sections reveal that zebrafish express one of the main BBB transports, Mdr1, in BECs over the course of development. 


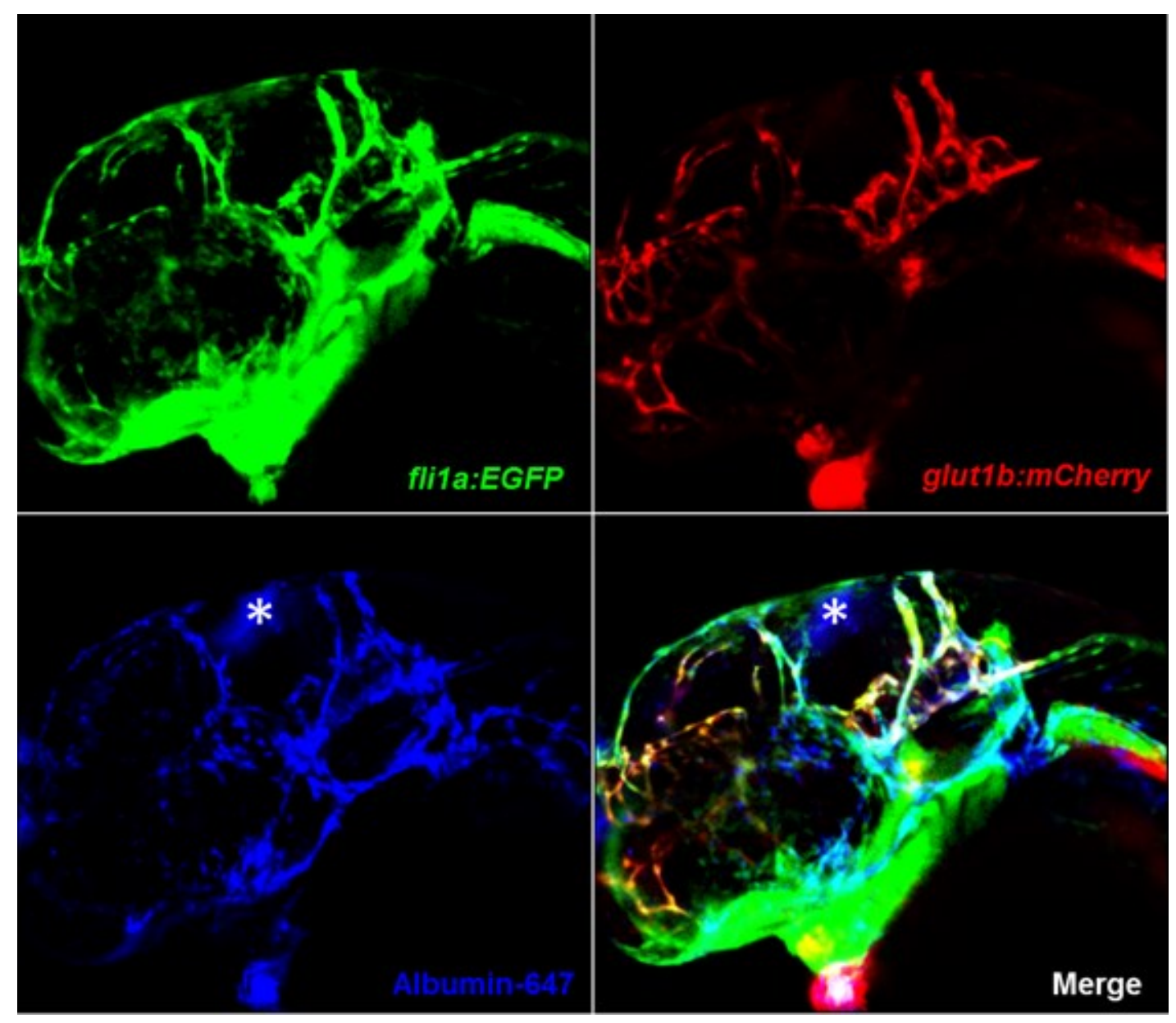

Figure 3-3. Newly formed vessels are impermeable to albumin

Tg(glut1b:mCherry;flila:EGFP) embryos were injected with Albumin conjugated to an Alexa Fluor 647 prior to CNS angiogenesis and visualized with live confocal imaging microscopy. These stills from the movie reveal that nascent glut $1 b$ positive blood vessels retain the large tracer when they migrate into the brain unlike the blood-CSF barrier (asterisks). 


\section{Signals that Regulate Barriergenesis}

Wnt Signaling. Wnt is a classical signaling pathway important for the initial stages of embryonic development but has also underlies the role in the health and differentiation of blood vessel morphogenesis ${ }^{101}$. Recent studies in mice have shown that canonical Wnt/ $\beta$-catenin signaling plays a critical role specifically in CNS angiogenesis and BBB development ${ }^{36,102}$. However, the onset of barriergenesis is difficult to resolve in these models due to embryonic lethality, the lack of normal CNS blood vessels, and the inability to visualize development in vivo. Therefore, to examine the effects of Wnt signaling on barrier formation in zebrafish, we crossed $T g$ (glut1b:mCherry; flila:EGFP) to $T g$ (hsp70l-1:GFP-axin1), a transgenic line that allows for heat shock-dependent expression of Axin1, a potent inhibitor of Wnt signaling $^{103}$. Modulation of heat shock parameters allowed for temporal and graded induction of Wnt inhibition. We performed heat shock for 60 minutes on the triple transgenic embryos and examined the effects at $3 \mathrm{dpf}$. While heat shock had no effect on control embryos without the axin-1 transgene (Figure 3-4C), we found that induction of Axin1 for 60 minutes completely inhibited angiogenesis and resulted in displaced mCherry expression (Figure 3-4G). These results are consistent with studies using conditional mouse knockouts of Wnt signaling, where Glut1 is ectopically expressed ${ }^{36}$.

To determine if we could dissect the contribution of Wnt signaling to both angiogenesis and barriergenesis, we reduced the duration of heat shock to 30 and 15 minutes. While 15 minute heat shock had no obvious effect (data not shown), we found that 30 minute heat shock was permissive for angiogenesis, but significantly inhibited mCherry expression (Figure 3-4K). These data indicate that there may be a Wnt signaling gradient that differs between angiogenesis and barriergenesis and that the level of inhibition may reflect differences in the expression of specific Wnt target genes. Differences in expression may explain why a canonical pathway has such a tissue specific phenotype. This interpretation of our results is also consistent with previous work demonstrating that specific Wnt ligands are spatiotemporally expressed throughout the developing mammalian brain and variably affect barriergenesis ${ }^{36}$.

VEGF, Shh, and BMP Signaling. As an additional proof-of-principle, we wanted to look further into signaling pathways important for mammalian barriergenesis in our zebrafish BBB model. With live imaging, we witnessed that barrier properties were present as soon as BECs migrated into brain tissue. Furthermore, we hypothesized that if CNS angiogenesis and barriergenesis were linked, then barrier properties should not be inducible in the absence of angiogenesis. This idea is commonly seen in BBB rodent studies, where knocking out genes important for barrier properties simultaneously disrupts CNS angiogenesis ${ }^{35,36}$. To test this interpretation, we chemically inhibited angiogenesis after the initial stages of development but before CNS angiogenesis occurred. At $24 \mathrm{hpf}$, we added the potent VEGF Receptor antagonists AV-951 (Tivozanib) and DMH4 to our BBB transgenic line and imaged the embryos after a 24 


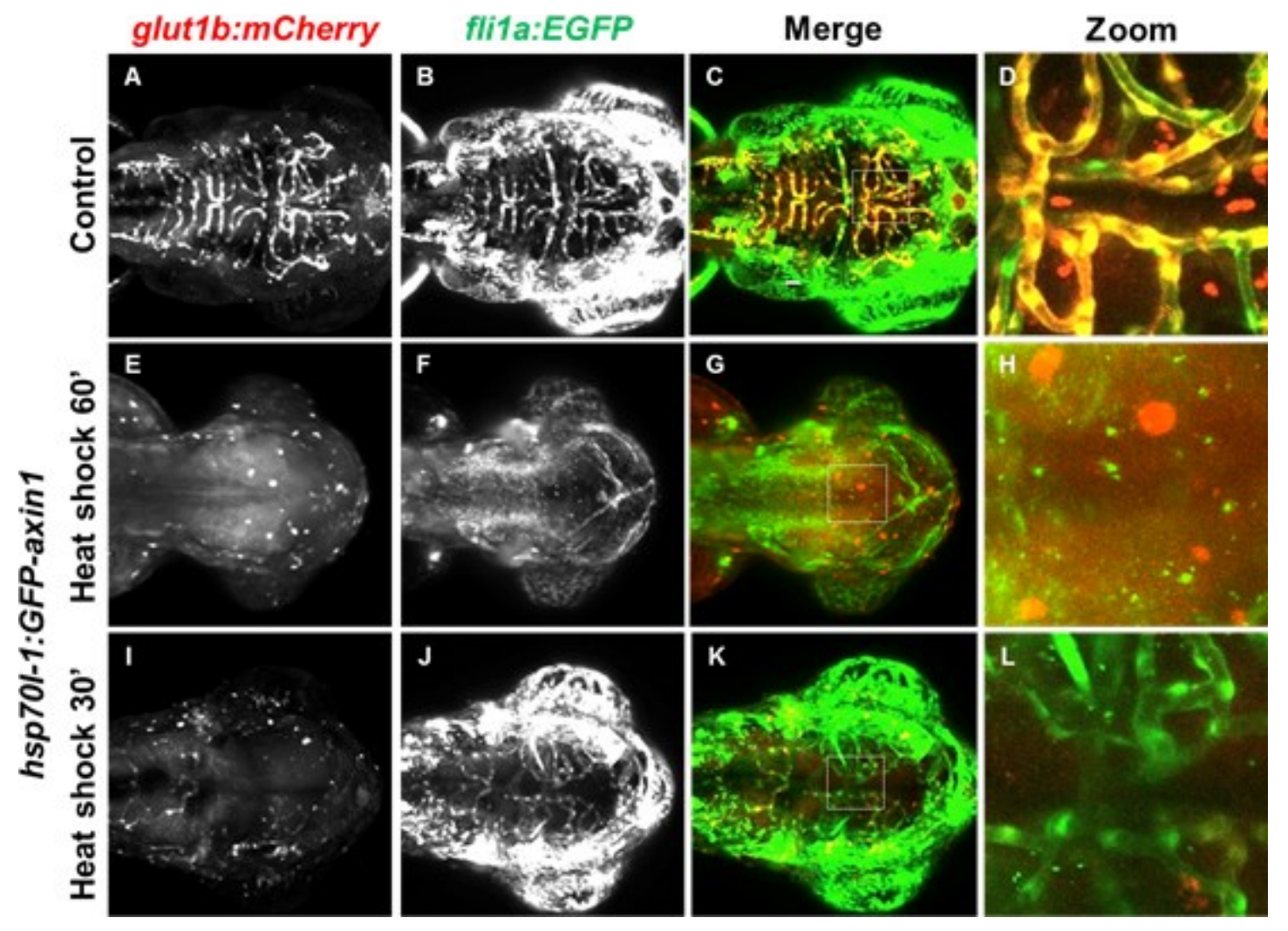

Figure 3-4. Levels of Wnt inhibition are permissive for angiogenesis but not barriergenesis

Transgenic hsp70I-1:GFP-axin1;glut1b:mCherry;flila:EGFP or

glut 1b:mCherry;flila:EGFP control embryos underwent heat shock at $24 \mathrm{hpf}$ at $38^{\circ} \mathrm{C}$ to deduce whether Wnt signaling was required for barrier properties in the developing zebrafish BBB. As seen in mice, 60 minute heat shock obliterated CNS angiogenesis and therefore barrier properties in the zebrafish BBB $(\mathbf{G})$. However, titrating Axin-1 induction back to a 30 minute heat shock allowed CNS angiogenesis to occur without barrier properties $(\mathbf{K})$, suggesting that Wnt signaling is required for the initiation of barrier formation but not CNS angiogenesis. 
hour exposure ${ }^{104,105}$. As expected, both compounds completely inhibited CNS angiogenesis (Figure 3-5). Surprisingly, the PHBCs, where hindbrain angiogenesis originates, strongly expressed mCherry, indicating that Glut1 can be induced in CNS vessels in the absence of angiogenesis (Figure 3-5). In contrast, previous work showed that Wnt signaling was necessary for GLUT1 expression in the perineural vascular plexus in the absence of CNS angiogenesis, thus implicating Wnt signaling in both angiogenesis and barriergenesis ${ }^{31}$. However, our data indicate that the acquisition of barrier properties, while dependent on Wnt signaling, is a distinct process from CNS angiogenesis and independent of VEGF signaling.

We next examined the role of Shh and BMP signaling on barriergenesis, because both pathways are known to be involved in vascular development ${ }^{106}$. In fact, a recent report demonstrated that brain astrocytes secrete Shh, BECs express Shh receptors, and concluded that the Shh pathway promotes BBB formation during embryonic development and adulthood ${ }^{33}$. To test this finding in zebrafish, we added the Shh pathway antagonists Cyclopamine to our transgenic line and found no obvious defects in either angiogenesis or barriergenesis (Figure 3-6G). We also found no effects with the BMP pathway antagonist DMH1 (Figure 3-6K). Thus, our results indicate that Shh and BMP signaling are not required for the initial induction of barrier properties in zebrafish.

Circulation and Glut1 Expression. We also wanted to determine if vascular circulation influenced the relationship between angiogenesis and barriergenesis. Recent studies have shown that shear stress plays a key role in the expression of important transporters and paracellular protein complexes that cause BEC differentiation ${ }^{107}$. Because zebrafish can survive early development in the absence of circulatory flow, we injected our BBB reporter line with a morpholino antisense oligonucleotide that targets cardiac troponin T2A (tnnt $2 a) \mathrm{mRNA}^{98}$. Embryos deficient for tnnt $2 a$ have a nonfunctional heart but little effect on CNS angiogenesis even without circulatory flow $^{108}$. Similarly, we found that tnnt $2 a$ morphants had thinner vessel lumens, most likely due to the lack of pressure from circulatory flow. However, we unexpectedly found that the tnnt $2 a$ morphants developed a CNS vasculature that still strongly expressed mCherry, suggesting that circulation may not be essential for barriergenesis (Figure 3-7). Because the vessel lumens were most likely collapsed, it is hard to determine quantitatively if tnnt $2 a$ morphants had an actual increase in glut $1 b$ expression as the mCherry signal may just be more concentrated due to the change in vessel structure (Figure 3-7H). If glut 1 b expression was actually increased, we suspect that hypoxia due to the loss of circulation might induce Glut1 overexpression at the BBB and therefore be relevant in neurological diseases such as stroke.

\section{Conclusion}

In conclusion, we report the first direct observation that BBB formation is initiated at the same time as CNS angiogenesis. Using time-lapse confocal microscopy, we showed that barriergenesis (i.e. the initiation of BBB development) occurs 


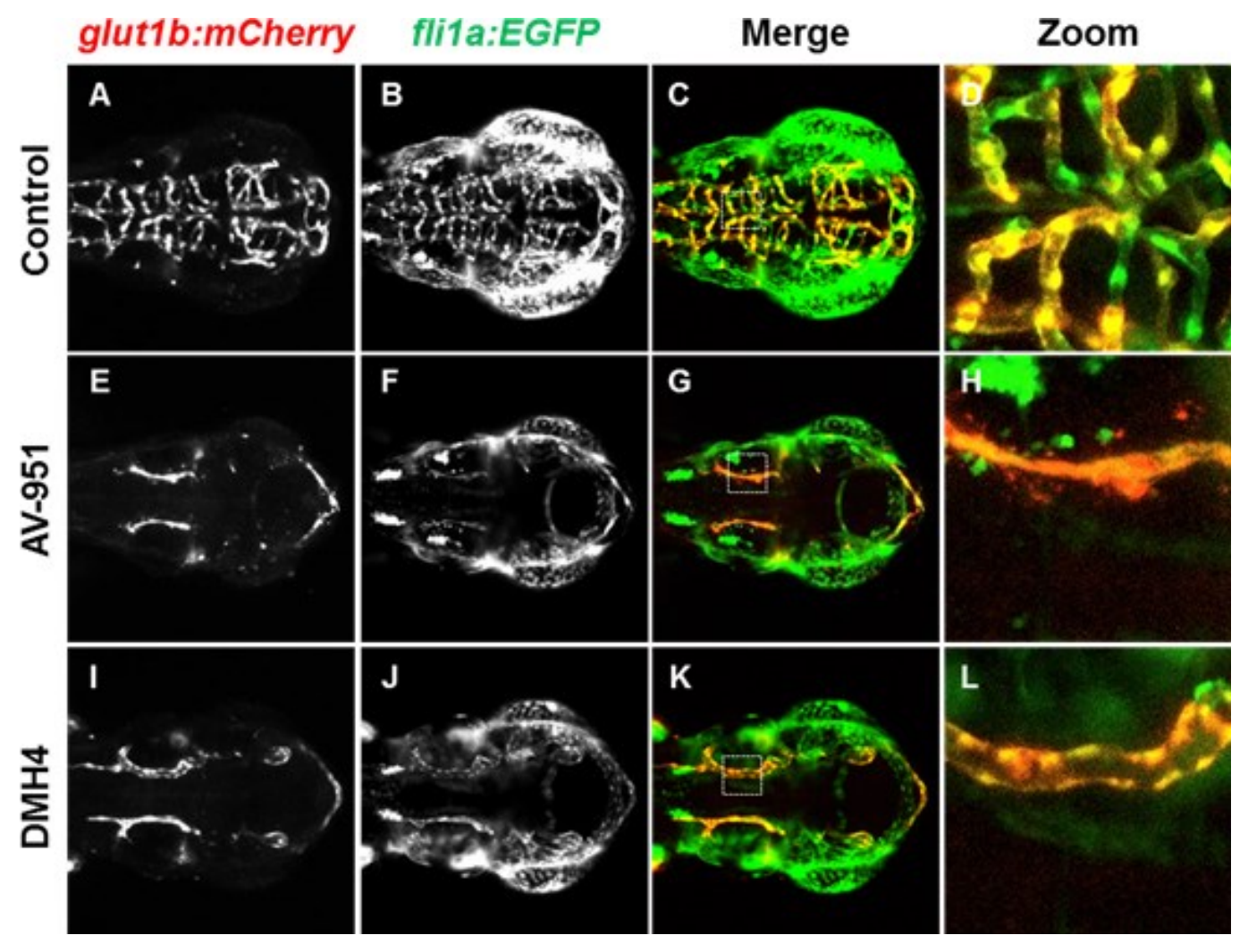

Figure 3-5. Angiogenesis is not required for barriergenesis to occur VEGFR-2 antagonists $1 \mu \mathrm{M} \mathrm{AV-951} \mathrm{and} 10 \mu \mathrm{M}$ DMH4 were added to $24 \mathrm{hpf}$ embryos before barriergenesis. As expected, both inhibitors prevented CNS angiogenesis but to our surprise, glut $1 b$ expression still persisted $(\mathbf{G}, \mathbf{K})$. 


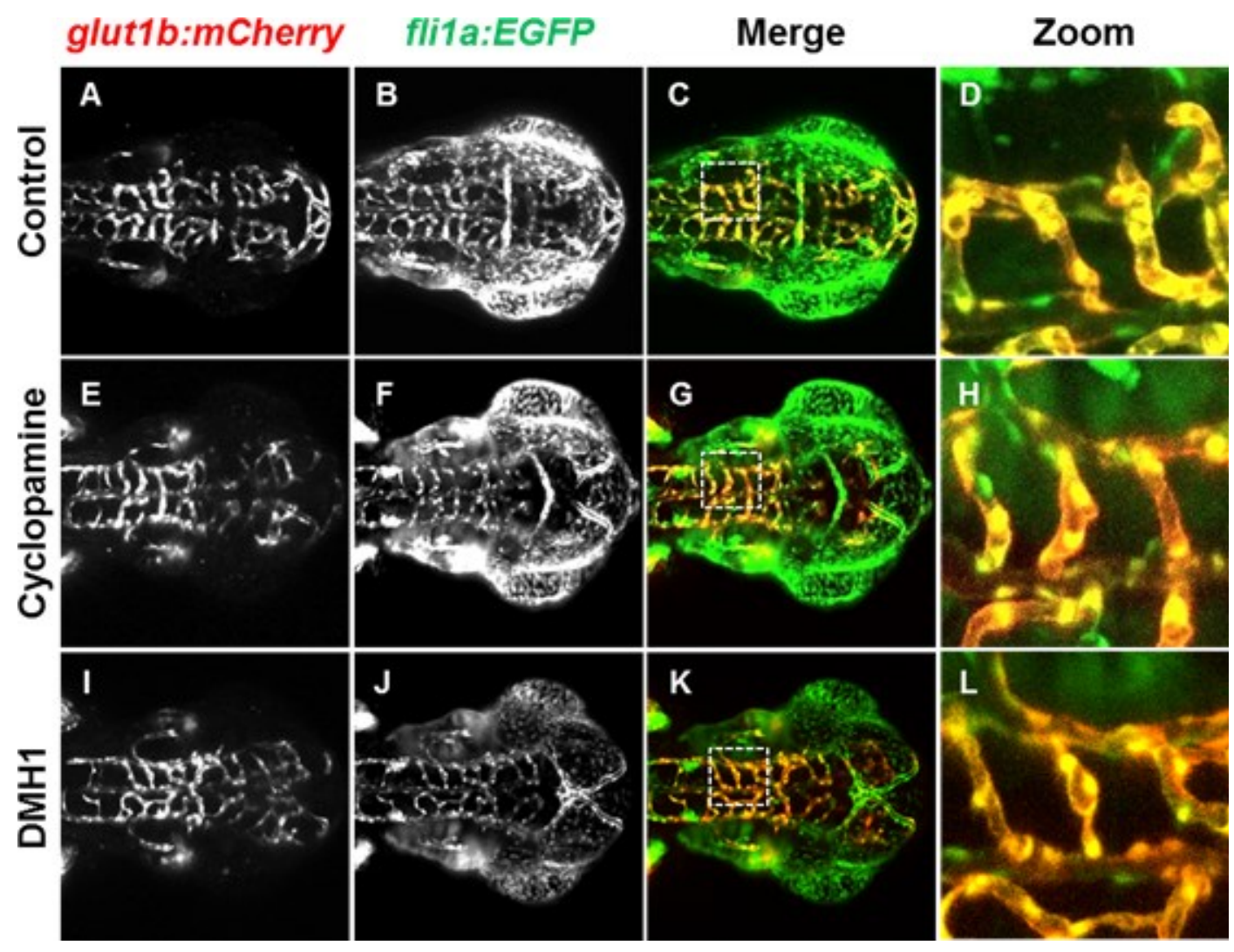

Figure 3-6. Shh and BMP signaling is not required for glutlb expression in the zebrafish blood-brain barrier

$50 \mu \mathrm{M}$ Cyclopamine and $10 \mu \mathrm{M}$ DMH1 were added to $24 \mathrm{hpf}$

$\mathrm{Tg}$ (glut1b:mCherry;flila:EGFP) to assess the effects of Shh and BMP signaling on barrier properties. Neither Cyclopamine $(\mathbf{G})$ nor DMH1 $(\mathbf{K})$ caused any effects on barriergenesis. 

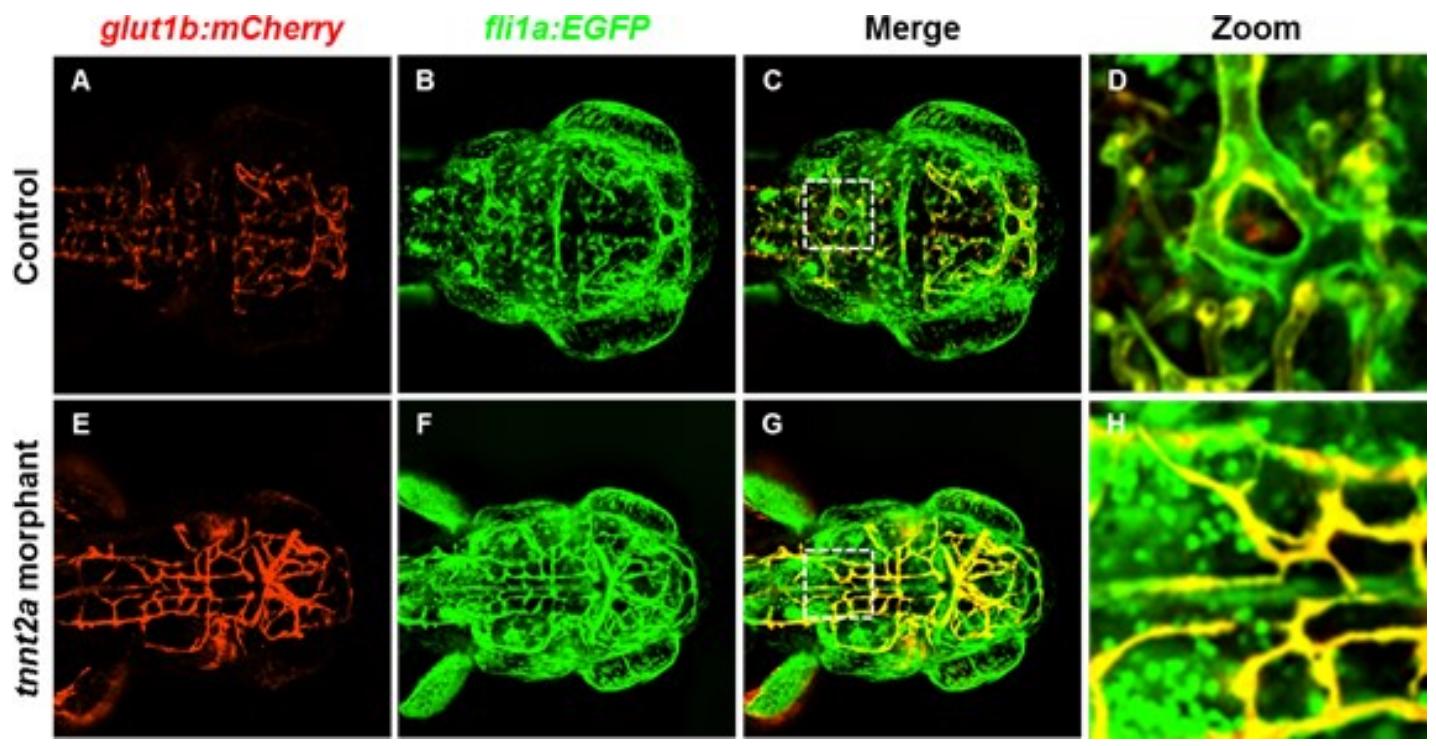

Figure 3-7. Circulation is not required for glut1b expression in the zebrafish blood-brain barrier

Single cell Tg (glut1b:mCherry;flila:EGFP) embryos were injected with the tnnt2a morpholino to assess the effects of shear stress on barrier properties. While heartbeat and circulation were obsolete, morphants still exhibited glut lb expression (E). Morphant vessels appeared brighter (E) compared to un-injected control siblings (A) but morphant vessels also appeared collapsed (H) compared to control vessels (D). 
immediately as new vessels sprout into the brain parenchyma and that these nascent blood vessels are functional. This is a novel finding that gives support for one of the two competing hypothesis in the field of BBB development. This result was possible with the advantages of our zebrafish model organism. Furthermore, using a combination of genetic and chemical approaches, we demonstrate that angiogenesis and barriergenesis, while occurring simultaneously, use different signaling mechanisms. We demonstrated that complete inhibition of canonical Wnt signaling blocks CNS angiogenesis, and that partial inhibition of Wnt signaling permits CNS angiogenesis but prohibits barriergenesis.

We expected Wnt signaling modulation to have an effect on mCherry expression since it has been shown that Glut1 expression is downstream of Wnt signaling. ${ }^{31,36,64}$ Additionally, we show that VEGFR antagonists block CNS angiogenesis, but do not prevent the induction of barrier properties in established blood vessels, indicating that barriergenesis is independent of VEGF signaling. Ultimately, these results show that CNS angiogenesis and barriergenesis occur simultaneously, but require distinct signals for proper formation. Our in vivo model provides a valuable tool for dissecting the complexities of the BBB that may ultimately lead to new treatment options for a variety of CNS diseases. 


\title{
CHAPTER 4. A FORWARD GENETIC SCREEN TO UNCOVER BLOOD- BRAIN BARRIER MUTANTS
}

\author{
Introduction
}

\section{Genetic Screen Strategies}

Genetic screening methods have been successful at dissecting signals important for developmental processes as well as their unknown functions. In molecular biology, there are two kinds of genetic screens that can be utilized, reverse and forward. In reverse genetic screens, a gene of interest is targeted to be deleted or knocked down and the phenotypic effects of this genetic ablation are observed. Common reverse genetic techniques include antisense oligonucleotide morpholinos, TILLING, retroviral-mediated mutagenesis, zinc finger nucleases, conditional bipartite systems for knocking out genes through transgenesis, TALENS, and the CRISPR/Cas9 system ${ }^{53-55}$. Forward genetics takes the opposite approach from reverse genetics. In a forward genetic screen, random point mutations are created in a founder generation, passed on to successive offspring, and mutations are discovered based on phenotypes that are identified. Forward genetic screens usually identify recessive mutations in a $\mathrm{F}_{3}$ generation, but haploid animals can be screened at an $\mathrm{F}_{2}$ generation to expedite screening for mutant phenotypes ${ }^{109,110}$. Therefore it is important to note the different starting and end results in these two kinds of assays. Reverse genetics is useful for screening for phenotypes if there is a gene of interest to be studied. Forward genetics proves to be a very useful and an unbiased strategy at identifying previously unknown pathways important for physiological processes.

\section{Significance of Forward Genetic Screens}

The first forward genetic screens were carried out in micro-organisms and helped identify the importance between genes and enzymes, gene structure, and even the genetic $\operatorname{code}^{53}$. Non-vertebrates such as the nematode (Caenorhabditis elegans) and fruit fly (Drosophila melanogaster) have also been used in forward genetics, elucidating loci involved in programmed cell death and cell polarity ${ }^{11,112}$. These findings were extremely significant to the field of biology and this research contributed to Nobel Prize awards in Medicine and Physiology. Important pathways that are conserved among various species have been identified in these model organisms, but their simplistic morphology limits what biological structures can be observed in these screens.

\section{Zebrafish and Forward Genetic Screens}

The zebrafish proved to be a useful vertebrate for forward genetics. It is possible to perform forward genetic assays in murine models, but intrauterine development, cost, 
and limiting size for pup liters make it difficult to study developmental processes in mammals $^{109}$. Because forward genetics is a high-throughput screening strategy, the fecundity of adults and transparent and rapid development of the embryo made zebrafish a suitable organism for the first genetic screens initiated by Streisinger and colleagues in the $1980 \mathrm{~s}^{113,114}$. These original zebrafish screens utilized gamma-ray irradiation until chemical mutagens were preferred in subsequent large-scale screens because of their ability to effectively generate germ-line mutations in a variety of loci ${ }^{48,49}$. The two first large-scale genetic screens in zebrafish in Boston and Tübingen identified over 6,000 mutant phenotypes, one third of these mutants being in developmental processes ${ }^{53}$. Since then, genes not only involved in embryogenesis but also in vision, cancer, the cardiovascular system, and brain development have been identified as well ${ }^{50,51,115-117}$.

\section{Treatment and Breeding Scheme in Zebrafish Forward Genetic Screens}

Typical forward genetic screens in zebrafish utilize breeding schemes for identification of homozygous recessive mutations that are inherited in a Mendelian manner in $\mathrm{F}_{3}$ diploid embryos (Figure 4-1). With this approach, founder males are treated with a chemical teratogen to introduce point mutations into their genome. Nethyl-N-nitrosourea (ENU), one of the most common chemical mutagens, is an alkylating agent that introduces random point mutations at loci throughout an organism ${ }^{118}$. The transfer of the ethyl group from ENU onto oxygen or nitrogen causes mis-pairing during DNA replication and after two rounds, causes a single base pair substitution and mutation $^{119}$.

The regimen and concentration of ENU used to treat zebrafish has been thoroughly studied, looking at survival rate, fertility, and frequency of mutation ${ }^{120,121}$. Efficiency of ENU mutagenesis is typically identified by a specific locus test, whereby ENU treated males are crossed to fish that are heterozygous for a known recessive mutation and their subsequent progeny are screened for the known mutant phenotype. Because ENU affects all of the cells in the adult male, only mutations generated in the pre-meiotic sperm cells passed onto non-mosaic $F_{1}$ progeny will be identified in further generations $^{121}$. After treated founder males recover, they are bred to wild-type females to produce a $F_{1}$ generation. The initial genetic screens reported that every $F_{1}$ individual possessed a genetic lesion that affected embryonic development. ${ }^{48,49,53} \mathrm{~F}_{1}$ progeny are then outcrossed to a polymorphic strain for future genetic cloning or in-crossed to other $F_{1}$ progeny to create the $F_{2}$ generation. Crossing $F_{1}$ progeny together will increase the number of screened genomes. After $\mathrm{F}_{2}$ families have been generated and raised to adulthood, pairs within $\mathrm{F}_{2}$ families are mated to identify the non-mosaic, recessive mutations in $\mathrm{F}_{3}$ offspring based on phenotypic inspection. The chance of finding a mutation in a particular mutagenized genome is determined by the number of crosses set up for that family. The probability of successfully finding a mutation in one family is shown in Equation 4-1 where $n$ is the number of successful matings between pairs of a given family ${ }^{48}$.

$$
P=\left(1-0.75^{n}\right)
$$




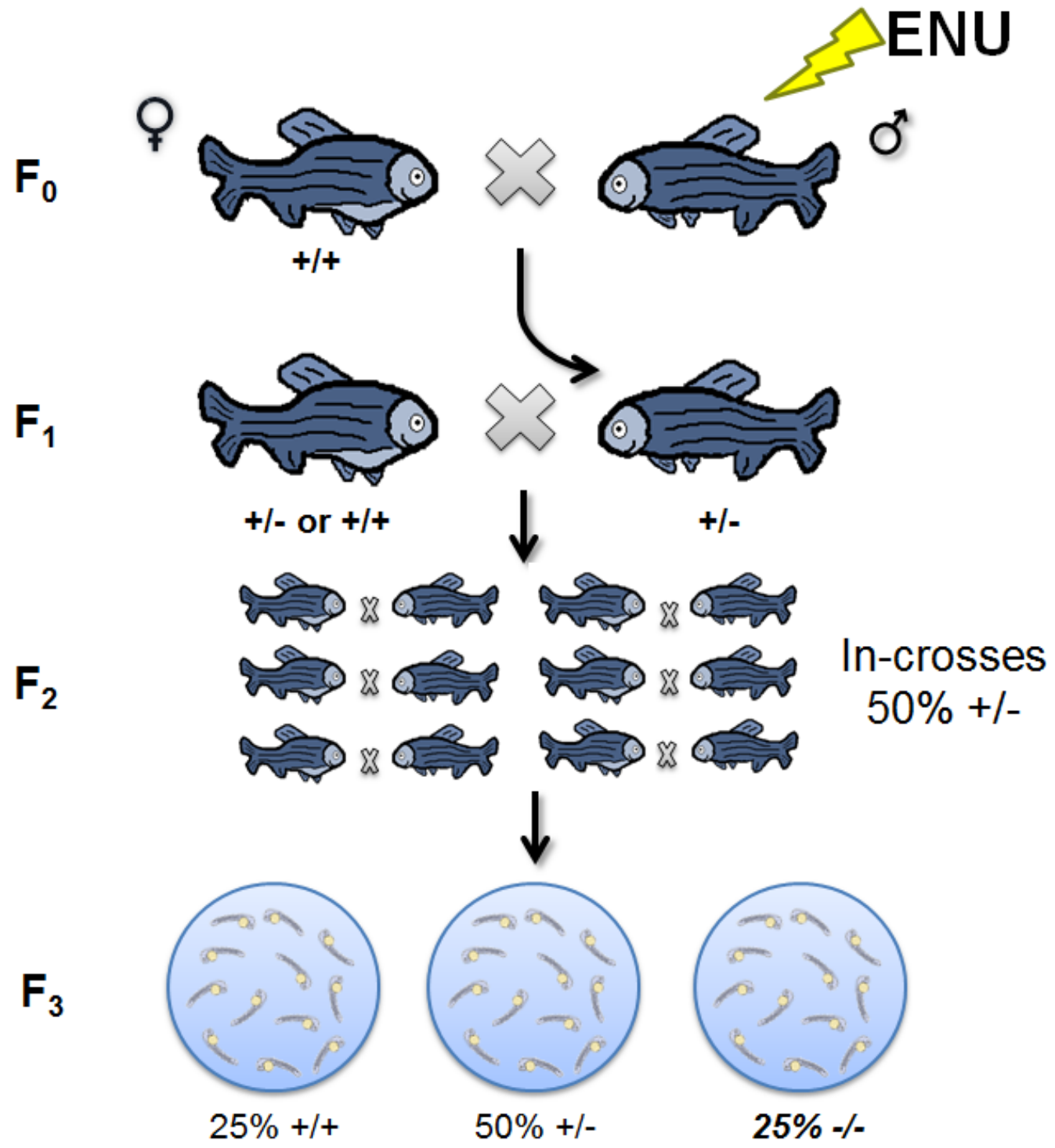

Figure 4-1. Breeding scheme for a zebrafish $\mathbf{F}_{\mathbf{3}}$ forward genetic screen Founder males are treated with ENU and out-crossed to wild-type females. The $\mathrm{F}_{1}$ generation is produced and offspring are heterozygous for germline mutations. In-crosses in the $F_{1}$ or outcrossing $F_{1}$ hets to wild-type fish will create $F_{2}$ families. $50 \%$ of the fish within a $F_{2}$ family are heterozygous for mutations from their $F_{1}$ parents. $F_{2}$ fish are crossed to one another to generate $F_{3}$ progeny. $F_{3}$ offspring will be screened for recessive mutations inherited in a Mendelian manner. Therefore, if a $\mathrm{F}_{3}$ clutch carries a recessive mutation, $25 \%$ of that clutch will present a phenotype. 
For example, if each family is mated to one another six times $(n=6)$, the probability of finding a mutation in that family will be $P=\left(1-0.75^{6}\right)$, where $P=0.82$ or $82 \%$ whereby only $18 \%$ of mutations for that family are lost. ${ }^{48}$ Rates of mutation after ENU treatment have been identified as 1.7 embryonic lethal mutation per single mutagenized genome ${ }^{120}$.

\section{Materials and Methods}

\section{ENU Treatment Materials}

For each isopack of ENU, a particular amount of solutions were prepared in a fume hood each week of treatment. The fume hood was covered in spill pads and the sash was concealed in aluminum foil to maintain a dark environment for the treated fish. $100 \mathrm{ml}$ of $10 \mathrm{mM} \mathrm{NaPO}_{4}$ buffer (pH 6.5), $1 \mathrm{~L}$ of ENU treatment water without tricaine (1 $\mathrm{mM} \mathrm{NaPO}_{4}\left(\mathrm{pH}\right.$ 6.5) made in fish system water), $3 \mathrm{~L} 1 \mathrm{mM} \mathrm{NaPO}_{4}$ buffer with tricaine for every tank of fish to be treated, $1 \mathrm{~mL}$ of $10 \mathrm{mM} \mathrm{NaPO}_{4}$ buffer (made in egg water) for spectrophotometric readings, two cuvettes, $5 \mathrm{~L}$ of inactivating solution (containing $20 \%$ $\mathrm{Na}_{2} \mathrm{~S}_{2} \mathrm{O}_{3}, 1 \% \mathrm{NaOH}$ ), and stock tricaine at $0.4 \%$ were prepared every week. Four larger mating tanks were used for treatment on each set of fish; one tank with an insert and the three others without. Two luer tip syringes and $50 \mathrm{~mL}$ conical tubes were needed for preparation of the ENU isopack

\section{ENU Preparation and Handling}

After the treatment solutions were prepared, a luer tip syringe was used to add 10 $\mathrm{mM} \mathrm{NaPO}_{4}$ buffer to the ENU isopack. A second syringe (with tip attached) was used for pressure buildup in the isopack because of the displaced gas that was produced as the buffer was added to the container. $60 \mathrm{~mL}$ of $10 \mathrm{mM} \mathrm{NaPO}_{4}$ buffer was first added followed by another $25 \mathrm{~mL}$ to total $85 \mathrm{~mL}$ of $10 \mathrm{mM} \mathrm{NaPO}_{4}$ buffer in an isopack. $20 \mathrm{~mL}$ of air was then carefully removed followed by $20 \mathrm{~mL}$ of ENU stock solution. This 20 $\mathrm{mL}$ aliquot was placed into a $50 \mathrm{~mL}$ conical tube and repeated so that each of the two conical tubes would have around $40 \mathrm{~mL}$ total between the two. ENU was mixed by pouring the tubes back and forth. To inactive any hazardous ENU spills and for clean-up, $5 \mathrm{~L}$ of inactivating solution containing $20 \% \mathrm{Na}_{2} \mathrm{~S}_{2} \mathrm{O}_{3}$ and $1 \% \mathrm{NaOH}$ was prepared.

\section{ENU Spectrophotometry Readings}

Because each isopack of ENU varied between bottles, the final concentration of ENU was calculated based on its optical density (OD) value with a spectrophotometer. Based on previously published screens, $3 \mathrm{mM}$ ENU was the desired concentration for each treatment ${ }^{120}$. ENU's molecular weight is 117.11 grams per mole and at a desired

molarity of $3 \mathrm{mM}$ that would constitute $0.351 \mathrm{mg} / \mathrm{ml}$ of ENU. For $1 \mathrm{mg} / \mathrm{ml}$ of ENU, at a 
spectrophotometer reading of $398 \mathrm{~nm}$, the OD is $0.72^{122}$. ENU was diluted 1:20 before being placed in a cuvette for readings on the spectrophotometer. Therefore, the dilution factor in $1000 \mathrm{ml}$ treatment water to total to $3 \mathrm{mM}$ per ENU isopack was calculated as shown in Equation 4-2.

$$
\frac{\text { [O.D. }}{\text { 398 value] X } 20 \text { (dilution factor) }}
$$

$1000 \mathrm{~mL}$ was divided by this dilution factor to decide what volume of ENU stock was needed to get a desired final concentration of $3 \mathrm{mM}$ ENU.

\section{ENU Treatment Paradigm and Fish Care}

ENU treatment was performed on 23 wild-type homozygous $\operatorname{Tg}($ glut $1 b$ : $m$ Cherry ;flila:EGPF) adult males with positive spawning and fertility histories. Eleven males and twelve males were separated in two initial treatment tanks, respectively, containing an insert and regular system water. After the OD of the ENU was calculated and $3 \mathrm{mM}$ ENU in system water was prepared, each set of fish was transferred with their insert into the $3 \mathrm{mM}$ ENU treatment water. Fish were bathed in this treatment water for 1 hour in a quiet and dark fume hood. After 1 hour of $3 \mathrm{mM}$ ENU treatment, the insert was carefully lifted and inserted into a subsequent water tank containing tricaine, a commonly used anesthetic for zebrafish. Tricaine was added as a means to help calm the fish down after ENU treatment. Fish were rinsed in this water for another hour in the quiet and dark. Fish were transferred to subsequent wash tanks for 1 hour at a time for 2 more rinses, totaling 1 ENU treatment bath and 3 rinses (Figure 4-2). After rinsing, fish remained in the last rinse tank overnight in the fume hood to help reduce death that may be caused by immediately transferring them back into system water ${ }^{123}$.

The fish's health was checked the following morning and then they were transferred back to the fish facility in a large tank with fresh tricaine wash water. A cardboard box was placed over the tank during transport to maintain a dark environment. Males would be set up for matings on Monday following ENU treatment and then treated with ENU on Thursday. This would help the fish get accustomed to mating for future breeding of the $F_{1}$ generation. ENU treatment was done once weekly over the course of three weeks.

\section{$F_{1}, F_{2}$, and $F_{3}$ Generations}

After three weeks of rest, the surviving ENU males, labeled A through I, were mated to wild-type $T g($ glut 1 b:mCherry ; flila:EGPF) females. These offspring were the $F_{1}$ generation and labeled with the letter of their original male father and spawn date. 625 fish were produced for the $F_{1}$ generation, were raised to a reproductive age, and then were mated to one another initially or wild-type $T g(g l u t 1 b: m$ Cherry;flila:EGFP) animals to produce $F_{2}$ families. $F_{2}$ families were labeled with a number starting at 001 , the letter $f$ 


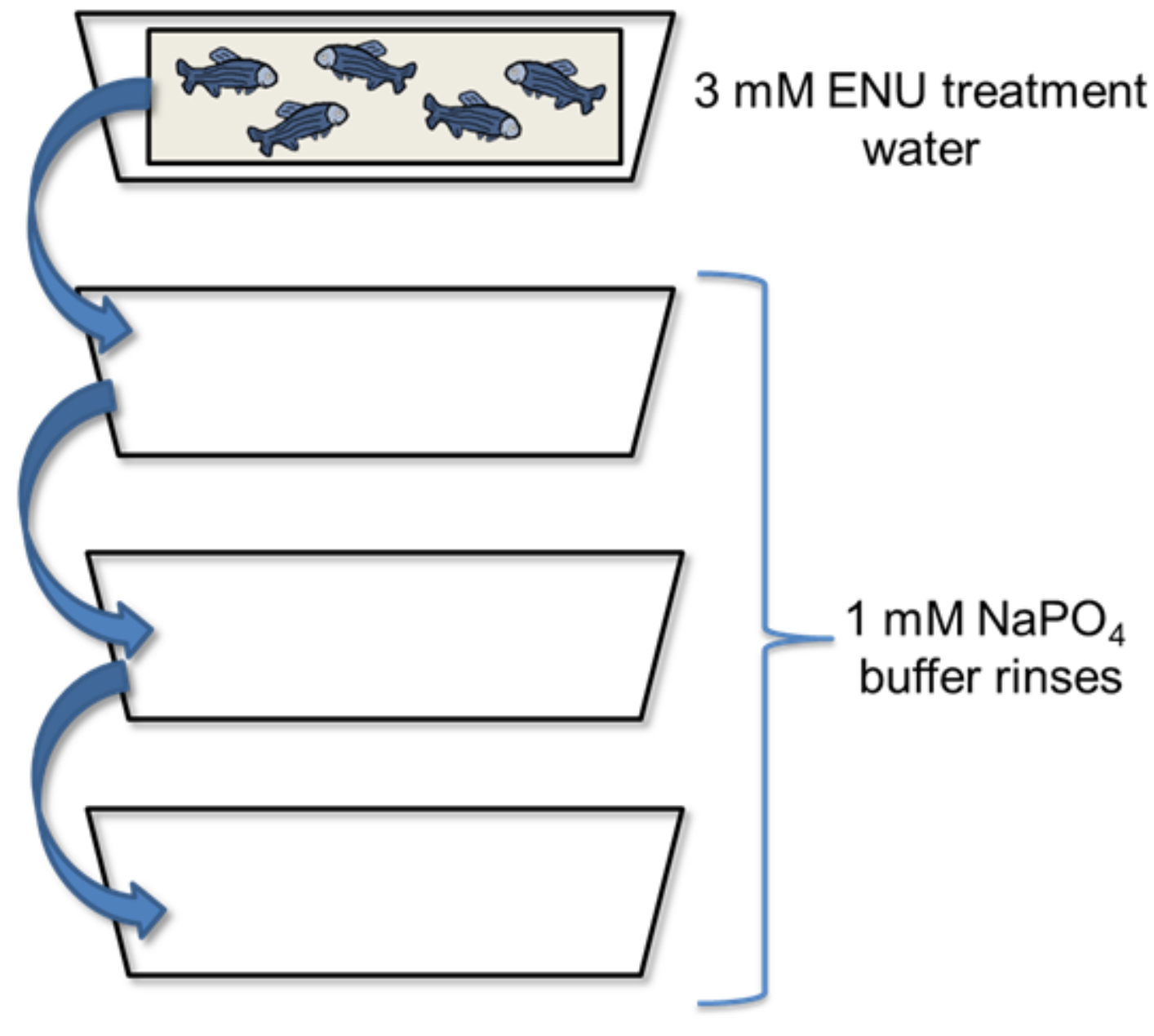

Figure 4-2. ENU treatment paradigm for male Tg(glut1b:mCherry;fli1a:EGFP) Founder males were treated with $3 \mathrm{mM}$ ENU once a week, for three weeks total. After each isopack of ENU was prepared and treatment water was made at $3 \mathrm{mM}$ ENU, fish were immersed in the ENU solution for 1 hour in a quiet and dark fume hood. After the treatment incubation, fish were transferred with an insert to subsequent tanks for washing in a $1 \mathrm{mM} \mathrm{NaPO}_{4}$ solution containing anesthetic. A total of 1 treatment and 3 washes were performed. After the last rinse, fish remained in the last tank over-night until they were transferred back to the fish facility. 
of the original $F_{0}$ male that gave rise to their $F_{1}$ parents, and their $F_{2}$ spawn date. Random matings between $F_{2}$ pairs generated $F_{3}$ progeny. $F_{2}$ pairs that spawned were isolated while their offspring were screened. These individual pairs were given an additional number after their $F_{2}$ family number as to track each pair. For example, the $6^{\text {th }}$ pair from family 022 was labeled as pair 22.6. $F_{3}$ progeny were labeled with the same number as their isolated $\mathrm{F}_{2}$ parents but their plates noted them as $\mathrm{F}_{3}$ and their spawn date. At least six successful paired matings within a $\mathrm{F}_{2}$ family were performed to optimize the chance of finding heterozygous pairs for a given mutation in each family.

\section{$F_{3}$ Progeny Screening}

$\mathrm{F}_{3}$ clutches were treated with PTU made in egg water to restrict pigmentation and make scoring fluorescence easier. The $\mathrm{F}_{3}$ generation was visually inspected under bright field during early development and assessed for GFP and mCherry expression in the BBB at $4 \mathrm{dpf}$. 4dpf was chosen because mCherry expression increases in intensity from $30 \mathrm{hpf}$ onward and is easily visualized by $4 \mathrm{dpf}$ without maternal contribution. mCherry was also present in the heart of transgenics which helped served as an internal control for mutations that would affect the folding and expression of just the mCherry protein. 150 $\mu \mathrm{L}$ of tricaine was added to each $\mathrm{F}_{3}$ dish before visual inspection under a Nikon SMZ 1500 stereoscope with a PRIOR Scientific Lumen 200 PRO Fluorescence Illumination System. Because we performed a forward genetic screen to identify recessive mutations, one-quarter of the $\mathrm{F}_{3}$ progeny would possess a mutant phenotype based on Mendelian probability if their $\mathrm{F}_{2}$ parents were heterozygous carriers for the same mutation. However, not all mutants were found at this ratio as phenotypic penetrance is not always Mendelian $^{124}$.

\section{Mutant Imaging}

4-5 dpf mutant and wild-type sibling larvae were anesthetized in 0.04\% tricaine made in PTU. After fish were asleep, they were embedded with $1.2 \%$ low-melting point agarose in a $35 \mathrm{~mm}$ glass bottom petri dish (MatTek). Dorsal z-stack confocal images were acquired in St. Jude Children's Research Hospital Cell and Tissue Imaging Facility on the Nikon C1Si microscope. Images were acquired with either a $20 \mathrm{X}$ lens or a $10 \mathrm{X}$ lens and a $1.5 \mathrm{X}$ zoom added in the Nikon C1Si software. Each slice was $4 \mu \mathrm{m}$ thick.

\section{Results and Discussion}

\section{Fish Survival and ENU Efficacy}

Of the 23 original treated males, 9 males survived and were assigned a letter A- I. This $39 \%$ survival rate falls slightly lower than the realm of what is expected with a 3 by $3 \mathrm{mM}$ ENU treatment regimen ${ }^{120}$. There are many factors that can induce death during 
and after ENU treatment, including environmental disturbance of light and sound during treatment, fish developing startling responses, an ineffective dose of anesthetic, hypersensitivity after treatment to noise and vibrations, and removing a dead fish during a hyper-sensitive period ${ }^{118}$. Even though we tried to avoid these issues and had a survival rate lower than $50 \%$, ENU teratogenicity was effective in subsequent generations.

To check for the potency of ENU treatment, males were mated to wild-type females and the offspring was observed for phenotypes with mosaic mutation. As seen in (Figure 4-3), just after one round of $3 \mathrm{mM}$ ENU treatment, a portion of offspring from treated males developed abnormally and no longer expressed EGFP from the transgenic background. ENU founder males were also crossed to Casper females to try and perform a specific locus test for the nacre and roy alleles. nacre mutants do not possess melanocytes and roy mutants do not possess iridophores ${ }^{125}$. After multiple rounds of mating, not enough embryos were spawned after each trial and no nacre or roy larvae were identified. After a month of recovery from the ENU treatment paradigm, males were bred to create the $\mathrm{F}_{1}$ generation. Around $625 \mathrm{~F}_{1}$ fish were generated from outcrossing males A-I. $188 \mathrm{~F}_{2}$ families were generated by $\mathrm{F}_{1}$ incrosses and $22 \mathrm{~F}_{2}$ families were generated from outcrossing a $\mathrm{F}_{1}$ to a wild-type $\mathrm{Tg}$ (glut lb:mCherry, flila:EGFP). Fewer $F_{2}$ families were generated from outcrossing an $F_{1}$ to wild-type transgenics as a means to cover more mutated genomes. From the $210 \mathrm{~F}_{2}$ families generated, 114 families survived but not all 114 families ended up having at least six pairs to mate or had equal sex ratios.

\section{BBB Mutants Discovered from Our Forward Genetic Screen}

Of the 68 screened families to date, 4 confirmed BBB mutant lines were identified and observed over the course of their development (Table 4-1). Some features scored in bright field included edema, necrosis, brain hemorrhage, and death. Mutants were identified by a change in barriergenesis by $T g($ glut $1 b: m C h e r r y)$ expression or a change in CNS angiogenesis development as indicated in $\operatorname{Tg}(f l i l a: E G F P)$. Because $\mathrm{Tg}$ (glut 1b:mCherry) transgenics also have heart expression, heart mCherry in scored mutants served as an internal control for mutations that affect the overall folding of the mCherry protein.

Families 040, 044, and 196 consisted of mutants with normal peripheral angiogenesis but $T g$ (glut 1b:mCherry) expression was absent or ectopic (Figure 4-4B, D, and F). These families had extreme cases of brain necrosis and a lack of CNS angiogenesis. Because brain necrosis was observed prior to and during barriergenesis, this suggests as it has been previously, that signals from the brain milieu are responsible for development of a healthy $\mathrm{BBB}^{32}$. Family 196 was also unique because mutants were identified with a reduction in $\operatorname{Tg}(f l i l a: E G F P)$ (Figure 4-4L ). This suggests that the mutation may affect vessel development health and delicate vessels may in turn affect barrier properties. 

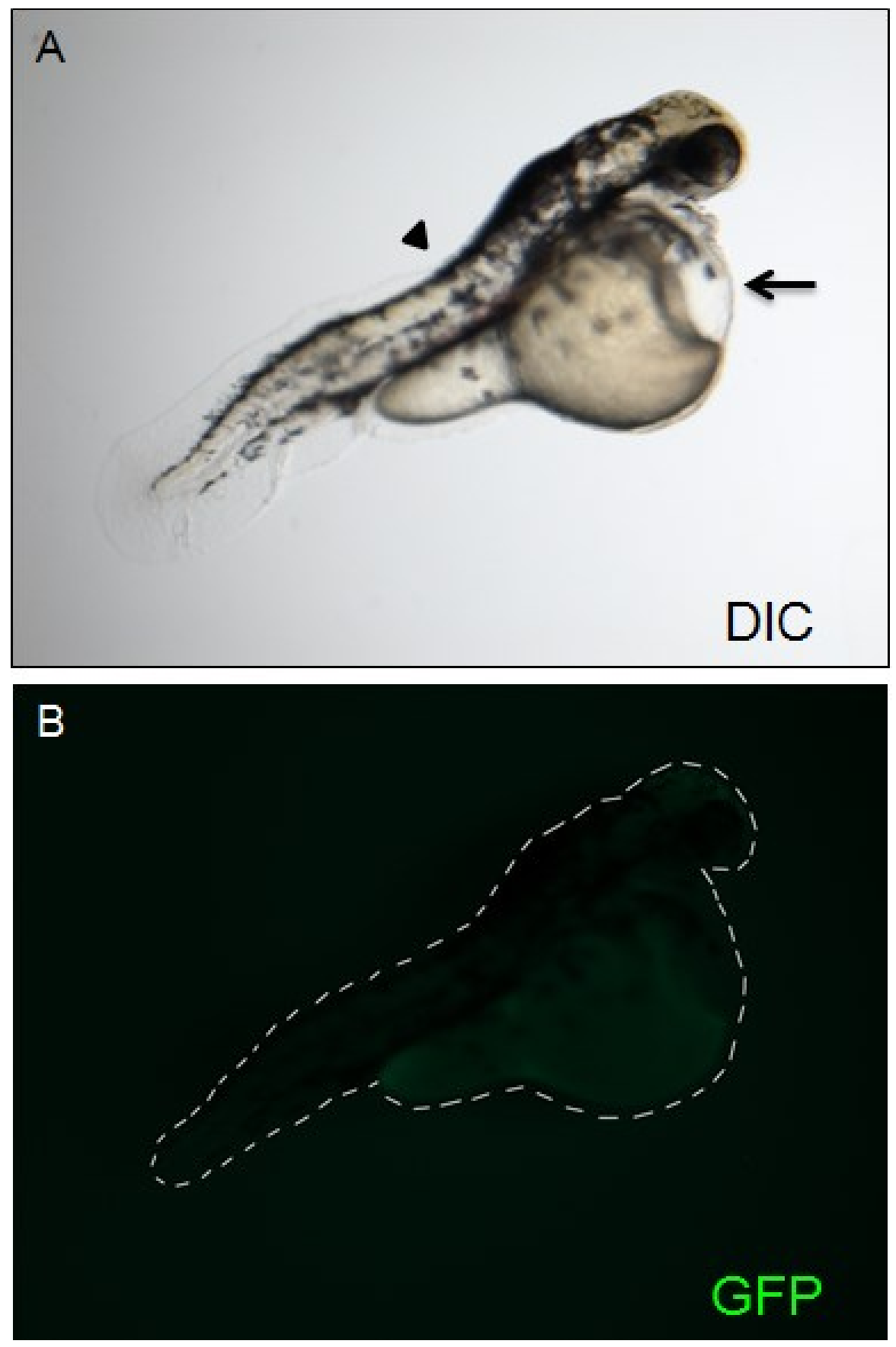

Figure 4-3. Effectiveness of $3 \mathrm{mM} \mathrm{ENU}$ after the $1^{\text {st }}$ round of ENU treatment After only one round of $3 \mathrm{mM}$ ENU treatment, a founder male out-crossed to a wild-type female produced a portion of developmentally abnormal embryos. (A) Brightfield image of embryo with heart edema (arrow) and bent back (arrow head). (B) Under GFP fluorescence, no flila:EGFP transgenic expression is visible. 
Table 4-1. Observation of mutant lines over the course of development

\begin{tabular}{ccccccc}
\hline Mutant Line & $\begin{array}{c}\text { Glut1 } \\
\text { expression }\end{array}$ & $\begin{array}{c}\text { BEC } \\
\text { development }\end{array}$ & $\begin{array}{c}\text { Hemorrhage } \\
\text { (dpf) }\end{array}$ & Necrosis & Edema & Neath (dpf) \\
\hline 22.6 & Highly reduced & Reduced & No & No & Heart & $6 \mathrm{dpf}$ \\
40.7 & $\begin{array}{c}\text { Ectopic; few } \\
\text { positive sprouts }\end{array}$ & $\begin{array}{c}\text { Minimal } \\
\text { sprouting }\end{array}$ & $\begin{array}{c}60 \% \text { mutants; } \\
2 \mathrm{dpf} \text { in brain }\end{array}$ & CNS & Heart and eye & $6-7 \mathrm{dpf}$ \\
196.1 & Highly reduced & None & 3 dpf in brain & CNS & CNS & Heart \\
\hline
\end{tabular}




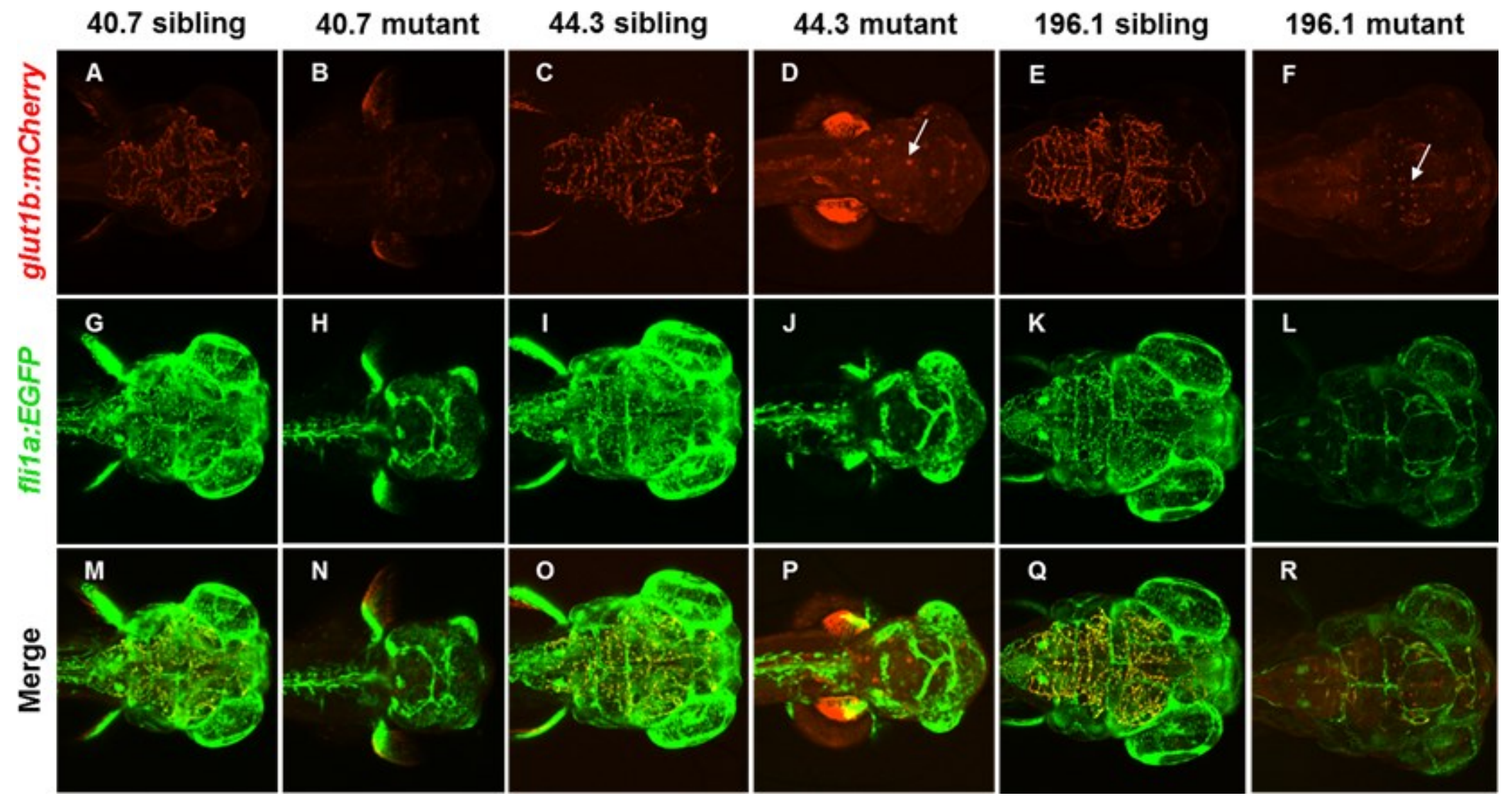

Figure 4-4. CNS angiogenesis mutants identified from the forward genetic screen

Families 040, 044, and 196 harbored mutants with very little to no CNS angiogenesis $(\mathbf{H}, \mathbf{J}$, and $\mathbf{L})$ but normal peripheral angiogenesis. 44.3 and 196.1 mutants had some mCherry expression but it was ectopically expressed (D and F arrows) compared to their wild-type sibling clutchmates $(\mathbf{C}$ and $\mathbf{E})$. 
Family 022 contained mutants with abnormal CNS angiogenesis but also a reduction in $\operatorname{Tg}$ (glut1b: $m$ Cherry) expression (Figure 4-5B). It has been shown in mice that genes important for BBB development affect GLUT1 expression and also CNS angiogenesis development ${ }^{35,36,126}$. It was ideal to find mutants that have a reduction in $T g$ (glut lb:mCherry) even though they still do not have completely normal CNS angiogenesis. Mutants like 22.6 further supports that barriergenesis can be affected separately from CNS angiogenesis even though these processes occur simultaneously.

\section{Conclusion}

We performed the first forward genetic screen to identify mutations that affect BBB development and maturation using our $T g($ glut 1 b: $m$ Cherry) reporter line. Survival of the $F_{1}$ larvae may have been low due to the mutations that were generated from the $F_{0}$ males. Dominant lethal mutations in the $\mathrm{F}_{1}$ generation may also eliminate the chance of finding heterozygotes in the $\mathrm{F}_{2}$ generation. Just like previous screens, we looked for recessive mutations transmitted to the $\mathrm{F}_{3}$ generation. Even though we were not able to confirm ENU efficiency with a specific locus test, we were able to show its efficacy after one round of treatment. To date in this small-scale screen, we identified 4 BBB mutants consisting of CNS angiogenesis mutants as well as a mutant that had a reduction in $T g$ (glut 1b:mCherry). These two classes of mutants suggest a genetic signature exists for BECs versus peripheral vessels as well as signals that affect barrier properties separately from CNS angiogenesis. Future endeavors include outcrossing mutant founder pairs to polymorphic strains, further characterization of each line, and cloning these mutants to identify regulators of CNS angiogenesis and barriergenesis. Ultimately, understanding the importance of these genes in barriergenesis could provide new therapeutic targets to modulate the BBB for drug delivery and prevent BBB breakdown in CNS diseases. 

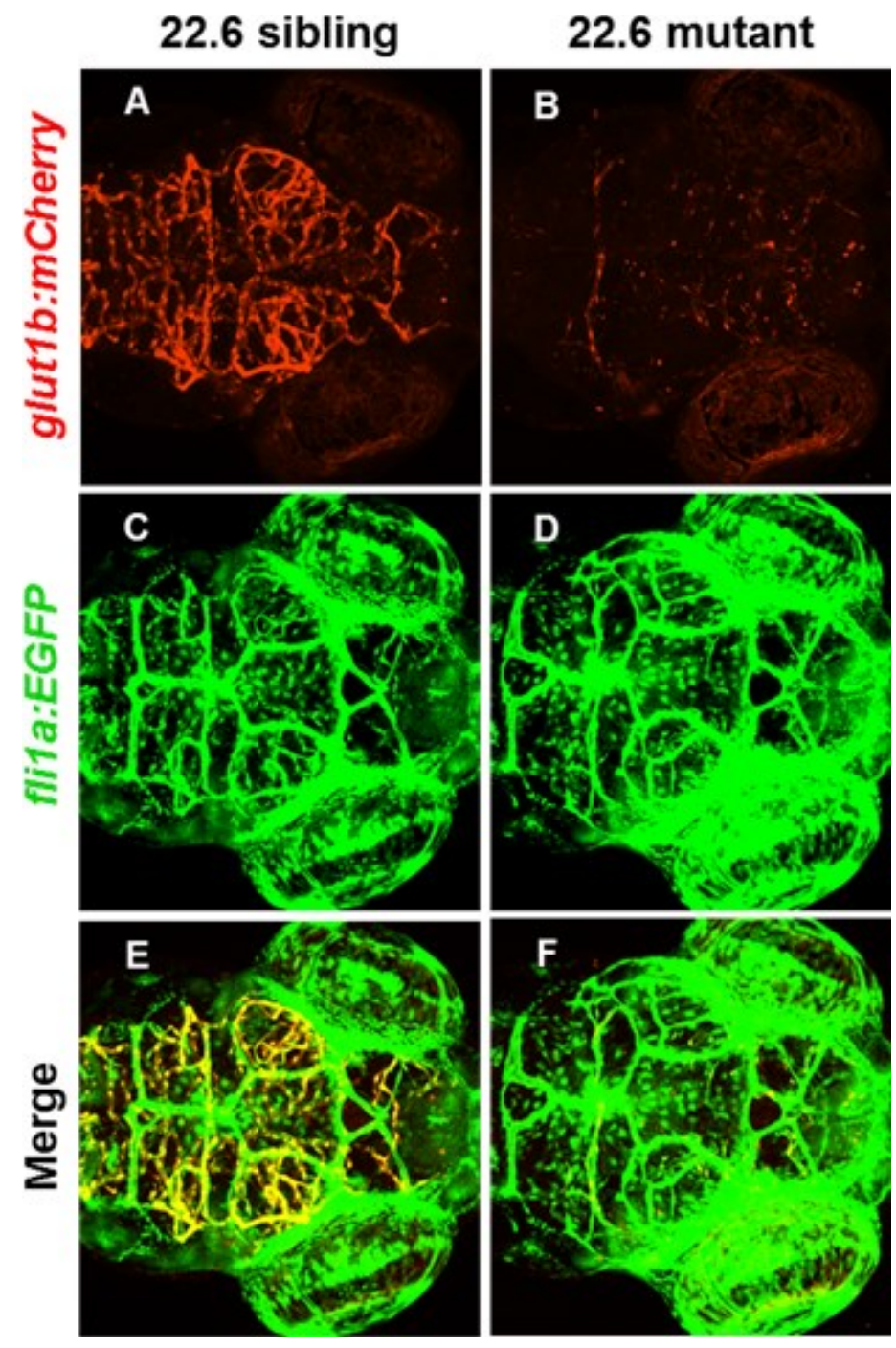

Figure 4-5. Tg(glut1b:mCherry) mutant and control sibling identified from the forward genetic screen

Family 022 harbored mutants with some degree of CNS angiogenesis (D) but a decrease in mCherry expression (B) compared to wild-type expression (A). 


\title{
CHAPTER 5. CLONING AND CHARACTERIZATION OF THE FIRST ZEBRAFISH BLOOD-BRAIN BARRIER MUTANT, 22.6
}

\author{
Introduction
}

\section{Positional Cloning}

The surge of zebrafish forward genetic screens in the 1990's advanced the identification of mutation induced phenotypes in the developing vertebrate ${ }^{48,49}$. Generating genetic maps with polymorphic markers allowed mutations to be identified through positional cloning ${ }^{127,128}$. Positional cloning, also known as bulk segregant analysis (BSA), utilizes the linkage of polymorphisms to mutant strains to identify the genomic region carrying the mutated loci that is causative for a mutant phenotype ${ }^{129,130}$. BSA therefore requires the outcross of the parental line carrying the genetic mutation to a polymorphic strain. Subsequently, heterozygous parents from this hybrid generation are identified and genomic DNA is isolated from individual mutant and wild-type siblings from in-crossing hybrid heterozygous parents. Different markers along the genome are analyzed between pools of the wild-type and mutant DNA until a region with fewer recombination events is uncovered. Because recombination events occur between loci that are farther apart, lower levels of recombination in mutant DNA pools will occur where the mutation is linked. A genomic interval that is linked to a mutation will have low amounts of recombination and co-segregate with the genetic strain where the mutation was initially introduced. The resolution of the linked interval is dependent on the number of meiosis analyzed, where 1 centiMorgen is 1 recombination event in every 100 meioses $^{130}$. Therefore, more meiosis are needed to define a smaller critical interval where a mutation will be mapped. After cloning a mutant, studies to perform characterization of the mutated gene, RNA rescue, genetic complementation, and phenocopy with gene knock down are typically implemented ${ }^{129}$. Positional cloning has been successful at identifying mutations responsible in a range of physiology including iron transport, gastrulation, and pigmentation genes conserved between zebrafish and humans ${ }^{131-133}$.

\section{Next Generation Sequencing}

BSA has been efficacious at isolating genes mutated in forward genetic screens but not every mutant in a forward genetic screen can be successfully cloned with this strategy $^{50,134}$. Positional cloning is quite time consuming and tedious if linked markers are not detected in the initial round of mapping. To circumvent these complications, next generation sequencing (NGS) strategies have increased the ease of cloning mutants discovered in zebrafish forward genetic screens ${ }^{135-137}$. As compared to BSA, fewer mutants are needed for NGS strategies to produce sufficient resolution for mutation identification $^{130}$. Pooled DNA from 20 mutants is adequate for NGS techniques as compared to a pool of 50 larvae that can resolve 1 centiMorgen in $\mathrm{BSA}^{130}$. 
There are various NGS methods, including Whole Genome Sequencing (WGS), Whole Exome Sequencing (WES), and RNA-seq. Each of these approaches has its own pros and cons depending on the type of data that can be generated. Even though the whole genome and its regulatory regions are analyzed in WGS, over $90 \%$ of the zebrafish genome is non-coding and most causative mutations usually occur in altering exons or splice sites, making WES a very attractive method ${ }^{130}$. RNA-seq allows the simultaneous analysis of expression differences but contains bias on the level and degree of tissue expression at the time RNA is extracted for use ${ }^{130,138}$. Such methods produce a large amount of computational data and the appropriate expertise and software is necessary to complete the analysis. Ultimately, NGS technology provides a new platform for efficient identification of mutants isolated in screens whereby prior genetic map linkage is not needed.

\section{Materials and Methods}

\section{Identifying the 22.6 Mutant Line}

22.6 mutants were identified in our forward genetic screen with $\operatorname{Tg}($ glut $1 b$ : $m$ Cherry; fli la:EGFP) for BBB mutants. In the 6th pair from the $22^{\text {nd }} \mathrm{F}_{2}$ family generated from ENU treated male $\mathrm{A}$, around one quarter of the $\mathrm{F}_{3}$ clutch had reduced mCherry expression on $4 \mathrm{dpf}$ compared to siblings. The 22.6 pair was crossed again to confirm Mendelian transmission of the recessively inherited phenotype that was seen in the first mating.

\section{Bulk Segregant Analysis}

In order to find genetic linkage to a chromosomal locus, 22.6 mutants were outcrossed to the polymorphic Tubingen Longfin (TL) strain. Upon sexual maturation, fish were in-crossed to find heterozygous carriers of the 22.6 mutant allele. Screening was performed based on phenotypic observation of the Tg (glut 1b: mCherry) transgene just as it was performed in the forward genetic screen to identify the original 22.6 pair. A total of 35 individual wild-type or mutant larvae were collected in $100 \%$ methanol. Fish were then digested in digest buffer with proteinase $\mathrm{K}$ and genomic DNA was isolated with 70 $\%$ ethanol. Pooled genomic DNA samples were diluted 1:10 in deionized water for a mapping PCR. Wild-type or mutant genomic DNA was added into an Invitrogen Accuprime Taq polymerase master mix. Master mix was added to a 384 well stock primer plate which contained all of the polymorphic marker primer pairs from the Massachusetts General Hospital (MGH) panel ${ }^{127}$. Samples were analyzed on a 4\% agarose gel to look for recombinants.

Primers for markers on linkage groups $3,11,14,22$, and 23 were ordered from Invitrogen for follow-up. Markers surrounding these from the MGH panel were selected from the ZFIN website and were also used for the follow-up PCR on individual wild-type 
siblings and mutant 22.6/TL. PCR master mixes and parameters were the same as with pooled DNA. Samples were analyzed again on a $4 \%$ agarose gel to look for recombinants.

\section{Exome Sequencing}

Genomic DNA was isolated from 40 wild-type sibling and mutant larvae with the Qiagen Mag Attract HMW DNA kit. Larvae were generated from five separate pairs from the original 022 family. Genomic DNA was analyzed for concentration and purity using a nanodrop spectrometer. This DNA was then submitted to the Hartwell Center at St. Jude Children's Research Hospital. Staff performed a double stranded DNA assay and captured $3 \mu \mathrm{g}$ of genomic DNA for sequence capture. The type of sequence capture used was the Agilent SureSelect Target Enrichment (in solution) Illumina Paired-End capture. The array/library used was the Agilent SureSelect XT Mouse methylseq-post capture pool.

From the raw sequencing reads, the quality control, mapping and variant calling were performed using CLC Genomic Workbench v6.5 (CLC Bio, Denmark). In brief, the reads were trimmed against the sequencing adapters, and only reads with a sequencing quality greater than 20 and a read length greater than $50 \mathrm{bp}$ were retained. The filtered reads were then aligned to the zebrafish reference genome sequence (Zv9 assembly, 2010), and the lists of single-nucleotide variants were generated. To identify the causative mutation responsible for the 22.6 phenotype, Michael Wang in the Hartwell Center compared the variants with those of wild type for mutant-specific homozygous mutations that have impact on proteins, including nonsense, missense and essential splice mutations. Michael identified a total of 44 mutant-specific mutations, two on chromosome 8 altering the amino acid sequences and one of them introduced premature stop codon in $g$-coupled protein receptor 124.

\section{Polymerase Chain Reaction (PCR) and Sequencing of Candidate 22.6 Mutations}

Primers were designed flanking the C2941T mutation (forward GCGGCGTCCCTCTAATCATA, and reverse ATGCCTAAACCGGTAGCTGT). Genomic DNA from 10 individual wild-type and 1022.6 mutants was diluted 1:10 for PCR. PCR was performed with Accuprime Taq polymerase. After PCR, products were analyzed on a $1 \%$ agarose gel before purification. $20 \mu \mathrm{l}$ of PCR product was purified with a Qiagen PCR purification kit. Samples were then nanodropped and $40 \mathrm{ng}$ of purified PCR was submitted for sequencing to St. Jude Children's Research Hospital Hartwell Center. An alignment of each individual wild-type or mutant sequence was performed against the gpr 124 cDNA (complementary DNA) reference sequence found on the Ensembl database (ENSDART00000112331) in the DNASTAR Lasergene 9 MegAlign software. Samples underwent the same processing for the candidate alpha-2B adrenoceptor (adra2b) with Ensembl sequence ENSDART00000018663 and primers 
flanking the A748G predicted mutation ( forward ATAAAGGTGGTGGCGAGTCA, and reverse GCCTTTGGTGTTCTTGCAGA ).

\section{Reverse Transcriptase Polymerase Chain Reaction (RT-PCR) of gpr124}

RT-PCR was performed on cDNA from 0- 5 dpf zebrafish. RNA was extracted from 10 embryos per tube with $50 \mu 1$ Trizol. Following Trizol extraction, RNA was recovered with a phenol chloroform spin, isopropanol and glycogen precipitation, and $75 \%$ ethanol wash. cDNA was synthesized after the Trizol RNA extraction by using an Invitrogen SuperScript III First Strand kit. The same primers from sequencing the candidate 22.6 mutation were used for RT-PCR using the Invitrogen Accuprime Taq polymerase system and a Biorad C1000 Thermal Cycler.

\section{IHC}

22.6 mutant and wild-type sibling larvae were collected at 3 or 4 dpf in a $1.5 \mathrm{~mL}$ tube on ice and fixed in $4 \%$ paraformaldehyde at $4{ }^{\circ} \mathrm{C}$ overnight. The next day, larvae were rinsed in phosphate buffered saline (PBS) three times for five minutes each on a nutator and then sunk in $30 \%$ sucrose at $4{ }^{\circ} \mathrm{C}$ overnight. After fish rose to the top at the sucrose's meniscus, they were removed and embedded in Optimal Cutting Temperature (OCT) medium in a plastic embedding mold. $12 \mu \mathrm{m}$ coronal frozen sections were cut on a Leica cryostat.

Sections were outlined with a pap pen to prevent the transfer of liquid between positive and negative control sides. Slides were then initially rinsed once in PBS, and then three times for five minutes each in PBST (PBS, 0.3\% TritonX 100) in a slide chamber on a belly dancer mixer. After rinsing, $5 \%$ goat serum with $1 \%$ bovine serum albumin block was added to sections for 1-3 hours. Blocking and antibody staining was performed in a humidity chamber made out of a sandwich box with wet paper towels. Primary antibodies rabbit anti Glut at 1 1:200 (Novus Bio), mouse anti Claudin5 at 1:200(Invitrogen), mouse anti GFP at 1:500(Invitrogen), and rabbit anti-GFP at 1:500 (Invitrogen) were diluted in blocking serum and then added to the bottom half of the sections and incubated at $4{ }^{\circ} \mathrm{C}$ overnight. The top part of the slide contained the negative control sections which were never stained with primary antibody but only secondary antibody. After incubation in primary antibody, slides were rinsed in PBST for 1 hour. Secondary antibodies were prepared in blocking serum (goat anti mouse 488, goat anti rabbit 488, goat anti mouse 555, and goat anti rabbit 555 all at 1:200, Invitrogen) and added to sections at room temperature for 2 hours. Slides were rinsed for another hour and then cover-slipped and sealed for imaging. 


\section{Cascade Blue, Rhodamine, and Fluorescein}

A minimum of five 22.6/TL mutant and wild-type siblings at $4 \mathrm{dpf}$ were injected IV (intravenous) with a 3,000 Da tracer called Cascade Blue (Invitrogen) or 10k Da Rhodamine into the common cardinal vein near the heart. Tracers were allowed to get into circulation for around an hour post-injection and then representative animals were imaged. Ten mutant or wild-type larvae from the same 22.6/TL clutches were incubated per well with $50 \mu \mathrm{M}$ Fluorescein (Fluka) made in PTU water for 30 minutes at room temperature in the dark. After incubation, larvae were briefly rinsed twelve times in clean egg water before embedding and imaging. All microinjections were performed using a PV820 Pneumatic PicoPump (WPI). Injection volumes were calculated using a $0.01 \mathrm{~mm}$ Stage Micrometer (Fisher Scientific).Larvae were anesthetized with $0.04 \%$ tricaine made in PTU water, embedded in 1.2\% low-melting point agarose on glass cover slip petri dishes, and were visualized on the Nikon AZ100 or Nikon C1Si confocal.

\section{Results and Discussion}

\section{Identification of the 22.6 Line}

Our first BBB mutant was identified from the sixth pair in family 022, originating from ENU-treated male A. Upon screening at $4 \mathrm{dpf}$ for changes in $\operatorname{Tg}$ (glut 1b:mCherry) expression, around a quarter of $\mathrm{F}_{3} 22.6$ larvae had little to no mCherry expression in the BBB compared to siblings. mCherry heart expression was unaffected, suggesting that this phenotype was not due to a mutation affecting the reporter gene itself. The mutant progeny from the original 22.6 pair did have a portion of embryos with brain hemorrhage on 4 dpf, a phenotype reported in the Gpr 124 mouse knockout ${ }^{35}$. However, when 22.6 was out-crossed to TL and new heterozygous carriers were identified, the brain hemorrhage phenotype was no longer seen.

\section{Cloning the 22.6 Mutation, gpr124}

To try and clone 22.6, we performed BSA on pooled genomic DNA from wildtype and mutant siblings while samples were correspondingly submitted for WES (Figure 5-1). Both experiments were implemented so two independent methods could confirm identification of the mutant allele.

We first performed the initial PCR on the full MGH panel. After the gels were analyzed, another round of PCR was performed with follow-up markers on individual larvae. Combing both techniques has successfully identified linkage and subsequent alleles in Xenopus tropicalis mutants ${ }^{134}$. While this is an ideal strategy, positional cloning is not always guaranteed to work for every mutant. For example, del Viso et al. (2012) were able to perform this dual approach on the ruby mutant but not on the grinch mutant $^{134}$. We were also unsuccessful at identifying a map position with BSA. However, 

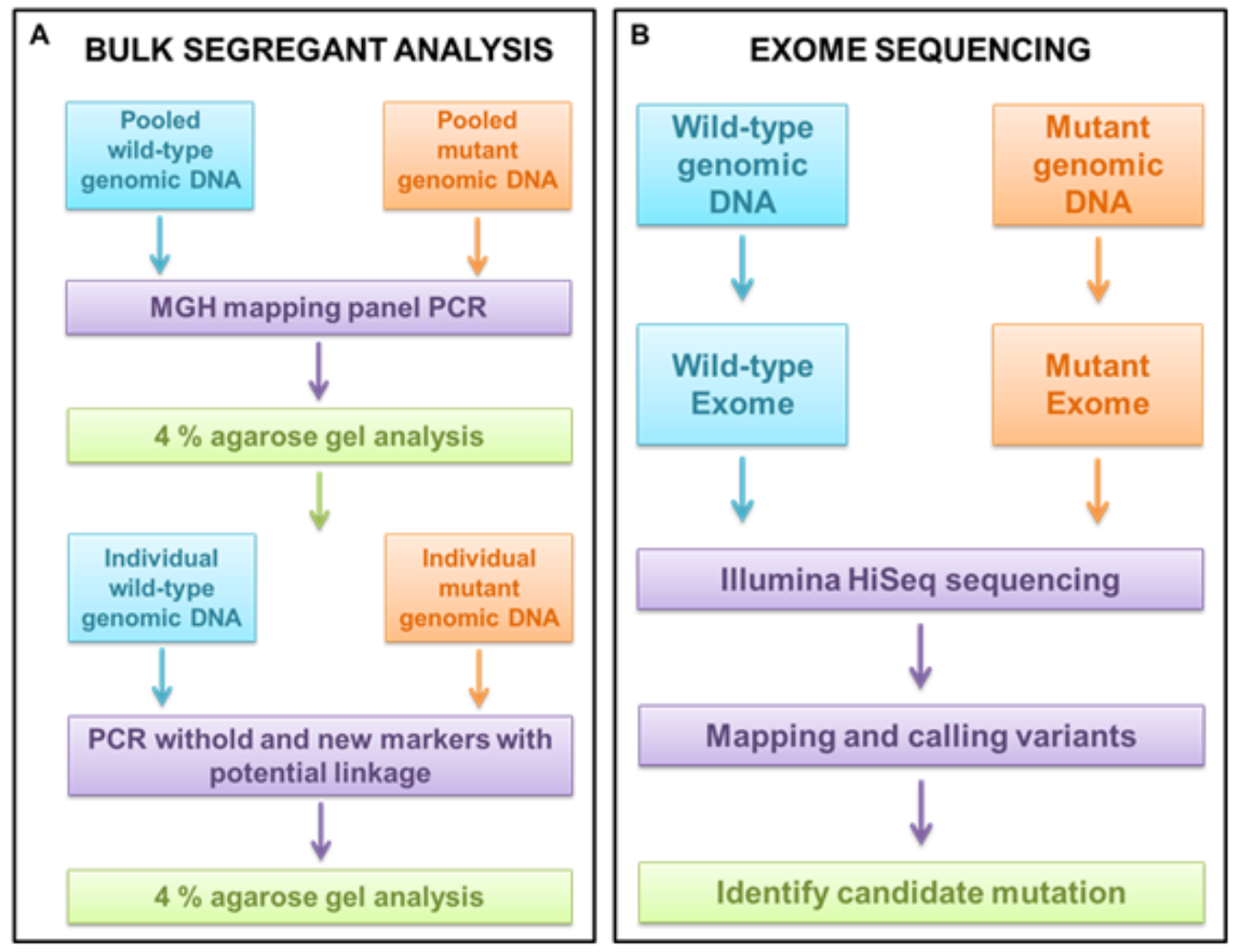

Figure 5-1. Workflow comparison of bulk segregant analysis and exome sequencing for cloning zebrafish mutants from a forward genetic screen

To clone mutants in our forward genetic screen, we wanted to utilize both bulk segregant analysis and exome sequencing. (A) For genetic mapping, larvae must be out-crossed to a polymorphic strain to identify linkage in the background which carried the mutation. For genetic mapping, we analyzed pools of wild-type and mutant genomic DNA with the MGH mapping panel. We then identified markers with possible linkage and obtained more primers surrounding those markers for a subsequent PCR. (B) We simultaneously submitted wild-type and mutant genomic DNA to the Hartwell Center at St. Jude Children's Research Hospital for exome sequencing. Hartwell Center scientists used the Agilent SureSelect Target Enrichment Illumina Paired-End capture and further analysis was done with the CLC Genomic Workbench v6.5 and zebrafish reference genome sequence (Zv9 assembly, 2010) to identify a candidate mutation. 
exome sequencing identified a guanine to adenosine point mutation at 39,116,104 bp on zebrafish linkage group 8 in the gpr 124 locus (Table 5-1). At the mRNA level, this causes a change from cysteine to thymidine at $2941 \mathrm{bp}$. Because the transcript is in the reverse orientation, this $\mathrm{C} 2941 \mathrm{~T}$ mutation causes a premature stop codon in the protein. $a d r a 2 b$ was also identified as a possible hit, but the point mutation in gpr 124 seemed worth following up on first since the base pair changed caused a premature stop in the protein and gpr124 has been implicated in BBB development (Table 5-1) ${ }^{34,35}$.

After identification of the point mutation, primers were designed flanking the C2941T mutation as well as the other candidate A748G mutation. Genomic DNA from the original fish used for exome sequencing was individually amplified with the flanking primers, the PCR products were purified, and the samples were then sent off for sequencing. Each individual wild-type larvae had a cysteine at position 2941 in gpr 124 cDNA however in each of the mutants, there was a thymidine at 2941 as seen from a subset of the alignments (Figure 5-2A). Furthermore, the A748G candidate in adra2b turned out to be a polymorphism, as a subset of wild-type siblings possessed a guanine at position 748 like the mutant samples (Figure 5-2B).

\section{Characterization of $\mathrm{gpr} 124^{-/}$Mutants}

IHC with BBB Markers. The BBB is commonly characterized for its physical, chemical, and functional properties. To assess these in the 22.6 mutant line, IHC and tracer injections were performed. Other groups have shown effects on tight junctions and transporters in BBB mutants ${ }^{35,36,102}$. Specifically, Cullen et al. (2011) showed that GLUT1 expression is disrupted in Gpr $124^{-/-}$mutant mice which agrees with the 22.6 mutant model from our BBB screen ${ }^{35}$. We knew that the mutation in gpr124 was predicted to cause a premature stop codon in the protein, but we wanted to know if the Glut1 protein was affected. To assess if Glut1 protein was affected in mutant BECs, IHC was performed on transverse sections from larval 22.6/TL mutants. As seen in sections from wild-type and mutants, the expression level of Glut1 protein was not different (Figure 5-3A). We also wanted to see if tight junctions were mis-expressed since hemorrhage was originally seen in 22.6 but not in 22.6/TL mutants. To do this, we performed IHC with a monoclonal Claudin 5 antibody on transverse sections from larval 22.6/TL mutants.

Table 5-1. Candidate mutations for the 22.6 line

\begin{tabular}{lllllll}
\hline $\begin{array}{l}\text { Gene } \\
\text { name }\end{array}$ & Chromosome & Position & Reference & Mutation & mRNA & Protein \\
\hline gpr124 & chr8 & 39116104 & $\mathrm{G}$ & $\mathrm{A}$ & $2941 \mathrm{C}>\mathrm{T}$ & p.Gln981* \\
adra $2 b$ & $\operatorname{chr} 8$ & 42259829 & $\mathrm{~T}$ & $\mathrm{C}$ & $748 \mathrm{~A}>\mathrm{G}$ & p.Thr250Ala \\
\hline
\end{tabular}


A
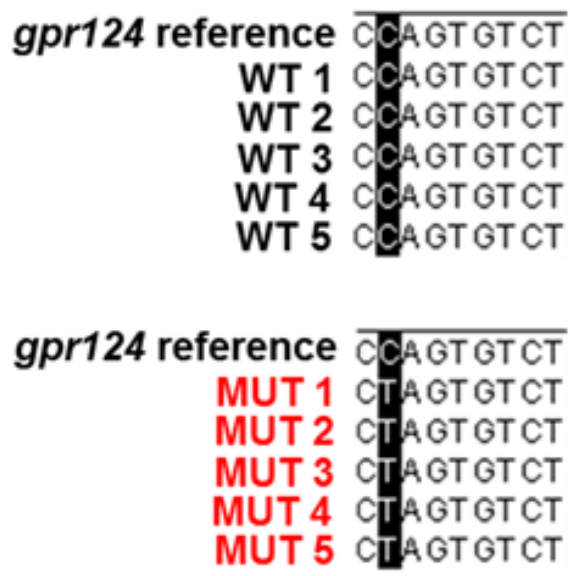

B

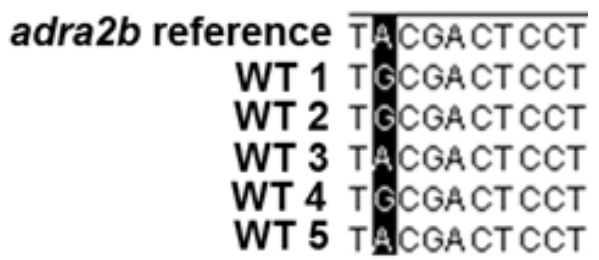

adra2b reference TACGACTCCT
MUT 1 TGCGACTCNT
MUT 2 TOCGACTCNT
MUT 3 TGNGACTCNT
MUT 4 TGCGACTCCT
MUT 5 TGCNACTCCA

Figure 5-2. Sequencing larvae for the 22.6 candidate gene mutations

(A) Primers flanking the C2941T mutation were used to amplify genomic DNA from individual larvae that were used for exome sequencing. A 1200 bp product was amplified from both wild-type and mutant samples. After PCR products were purified, they were sent off for sequencing with the same primers used in the PCR amplification. Sample sequences were aligned to a gpr 124 cDNA reference sequence. Alignments revealed that, as predicted, the wild-type samples possessed a $\mathrm{C}$ at position 2941 while each of the mutants possessed a T. (B) Similarly, PCR was performed with primers flanking the predicted $\mathrm{A} 748 \mathrm{G}$ adra $2 \mathrm{~b}$ mutation. The $971 \mathrm{bp}$ PCR product was sent off for sequencing with the same PCR primers. As confirmation that gpr124 was mutated and not $a d r a 2 b$ in our 22.6 line, alignment against $a d r a 2 b$ cDNA revealed that the A748G base change was a polymorphism. While all mutant samples contained a $\mathrm{G}$ at position 748, some wild-type samples contained a $\mathrm{G}$ at position 748 as well. 
A
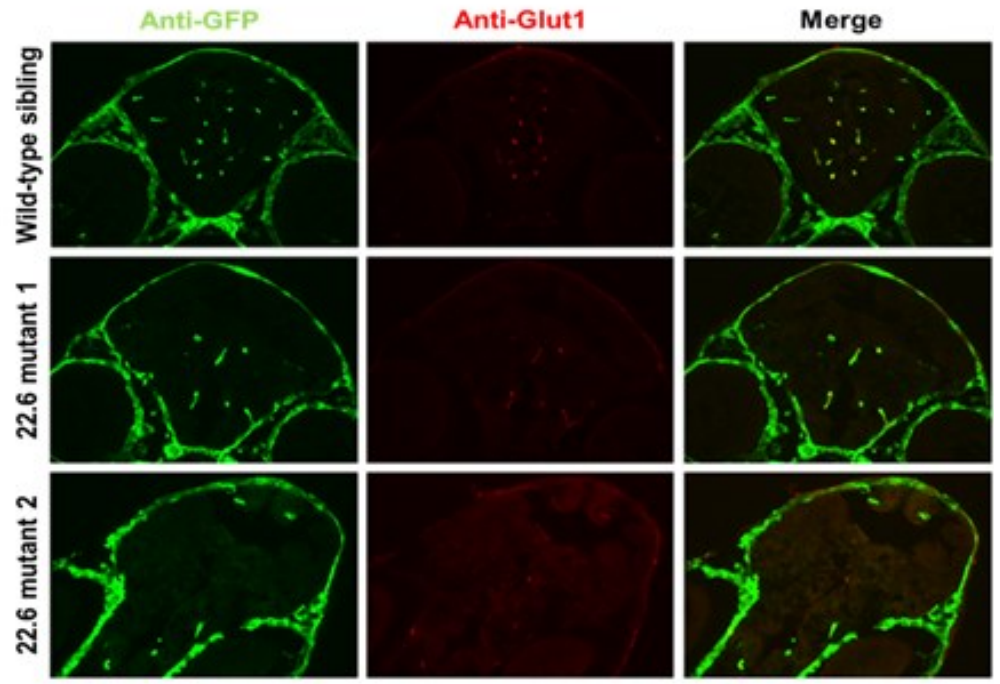

B
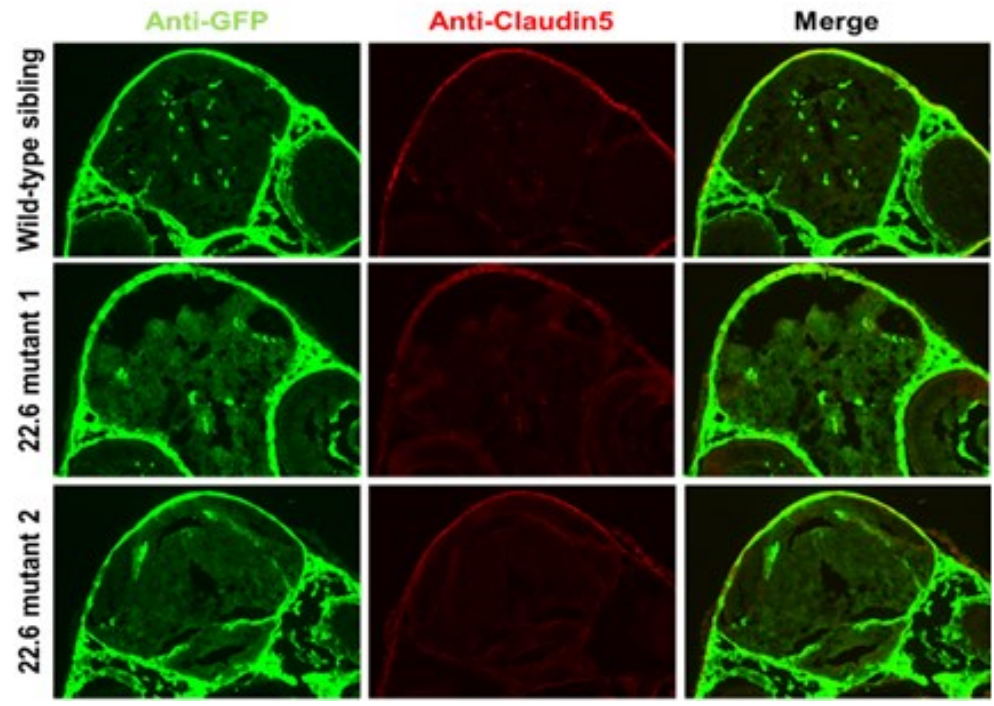

Figure 5-3. Immunohistochemical analysis of BBB markers Glut1 and Claudin5 in 22.6 mutants and wild-type siblings

Antibody staining for Glut1(A) and Claudin5(B) proteins was performed to identify any differences between 22.6 mutant and wild-type sibling BBB protein marker expression. Staining revealed that at the protein level, there was no difference in the intensity of Glut1 or Claudin5 expression even though there are fewer BECs in the mutants as seen with GFP staining. 
Just as with the Glut1 protein, we did not see any difference at the protein level with the Claudin 5 antibody in 22.6 mutants (Figure 5-3B). The expression of Claudin 5 may not have been affected due to compensation from other tight junction proteins. Also, Claudin $5^{-/-}$mice do not experience brain hemorrhage but a "size-selective" leakiness when injected with tracers suggesting lack of Claudin5 does not cause brain hemorrhages but a selective opening of the barrier ${ }^{22}$. Furthermore, signaling pathways important for barriergenesis, like Wnt signaling, affect Claudin5 expression but not Occludin or ZO-1 demonstrating distinct target genes among proteins that have similar functions in the barrier $^{36,102}$.

Functional Studies with Tracers. We no longer saw brain hemorrhages in the outcrossed 22.6/TL line but still wanted to see if mutant vessels were leakier than wildtype since $G$ pr $124^{-/-}$mice have brain hemorrhages ${ }^{35}$. To assess the function of the BBB in 22.6/TL mutants, fluorescent tracers were injected into circulation or bathed on 22.6/TL mutants and wild-type siblings. After IV injection, Cascade Blue, a 3,000 Da tracer leaked into the mutant brain greater than in wild-type larvae (Figure 5-4). We then wanted to see if this leakiness would still hold true with a tracer smaller and larger than Cascade Blue. The BBB of 22.6/TL also showed leakage with $10 \mathrm{kDa}$ Rhodamine and 332 Da Fluorescein tracers (Figure 5-5). While the 22.6/TL mutants did not have hemorrhages, we still saw leakage with tracers smaller than large plasma proteins like the $78 \mathrm{kDa}$ Vitamin-D binding protein which is a member of the zebrafish albumin family ${ }^{62}$. Interestingly, while Rhodamine gets into the brain parenchyma of $22.6 / \mathrm{TL}$, it appears as if the blood-CSF barrier is still intact (Figure 5-5A arrows).

\section{Conclusion}

The 22.6 mutant line was the first mutant identified from our BBB forward genetic screen. To clone this line, we simultaneously performed BSA and NGS experiments. Like other groups, we did not get a successful map position with BSA but were able to clone 22.6 with WES $^{134}$. WES revealed a point mutation in the gpr 124 allele which in turn was predicted to cause a premature stop codon in the protein sequence. Gpr124 has already been shown to be important for BBB development in mammals and therefore cloning this mutant validates the authenticity of our screen to find BBB mutants ${ }^{34,35,126}$. In order to investigate the structure of the $22.6 \mathrm{BBB}$, we looked at Claudin5 expression in mutant BECs. We did not notice a change in protein levels for Claudin5 in mutants compared to wild-type siblings. We also observed a sizeselective leakiness in mutants with a 3000 Da Cascade Blue but not a $10 \mathrm{kDa}$ Rhodamine tracer. While the protein level of Glutldid not dramatically decrease in sections, it is hard to quantify expression level from sections. Also, Tg(glut 1b:mCherry) was not completely absent in the 22.6 line as seen by confocal microscopy. Ultimately, cloning a gpr 124 zebrafish mutant will provide the BBB community with another tool to study barriergenesis. Furthermore, g-coupled protein receptors are highly targeted in drug discovery and finding the gpr124 ligand may now be easier with this zebrafish mutant and model $^{139}$ 


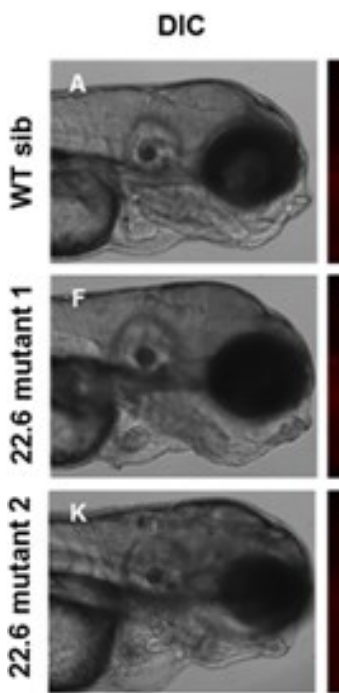

mCherry
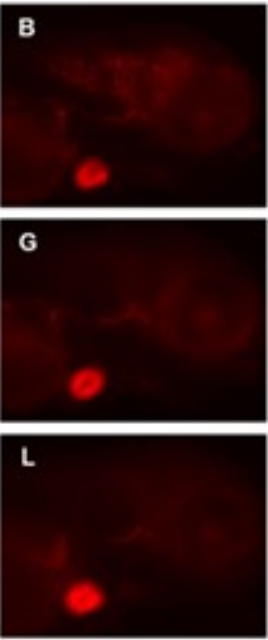
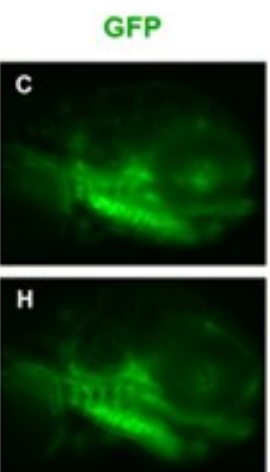

M
Cascade Blue
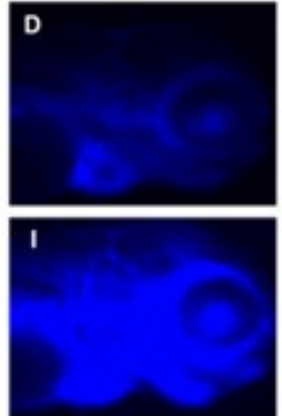

N

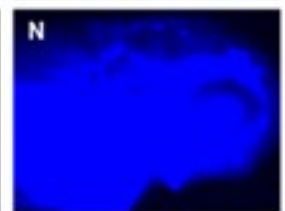

Merge
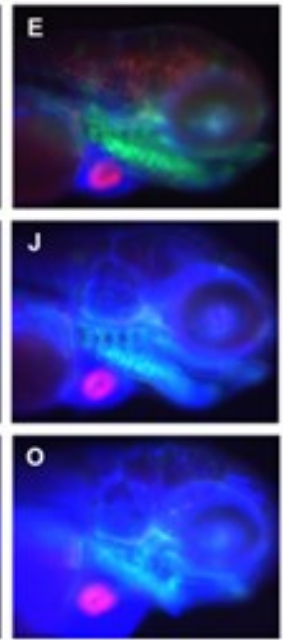

Figure 5-4. 22.6/TL mutants appear "leaky" after IV injections with Cascade Blue

4 dpf Wild-type and 22.6 mutant siblings were injected with Cascade Blue, a 3,000 Da tracer into the common cardinal vein. After tracers were in circulation for 1 hour, larvae were imaged on the Nikon AZ100. As compared to wild-type (D), two representative mutants had increased fluorescence in the blue channel (I, N). This "leakier" 22.6 phenotype may be attributed to the fact that there are less vessels in the mutant $(\mathbf{H}, \mathbf{M})$ compared to wild-type siblings $(\mathbf{C})$ as captured with GFP. 
A

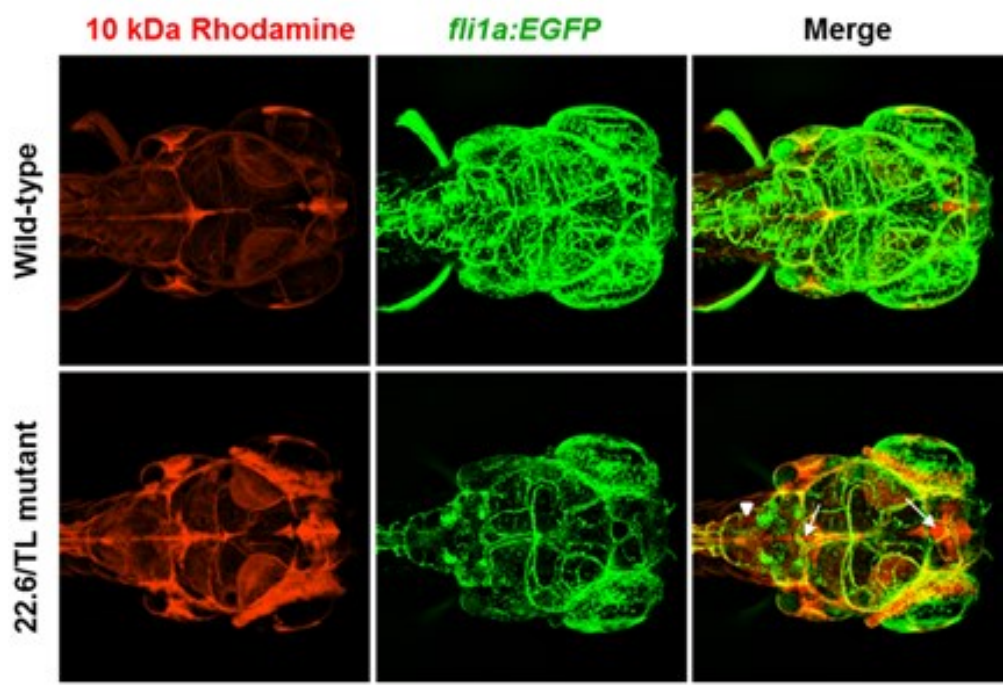

B

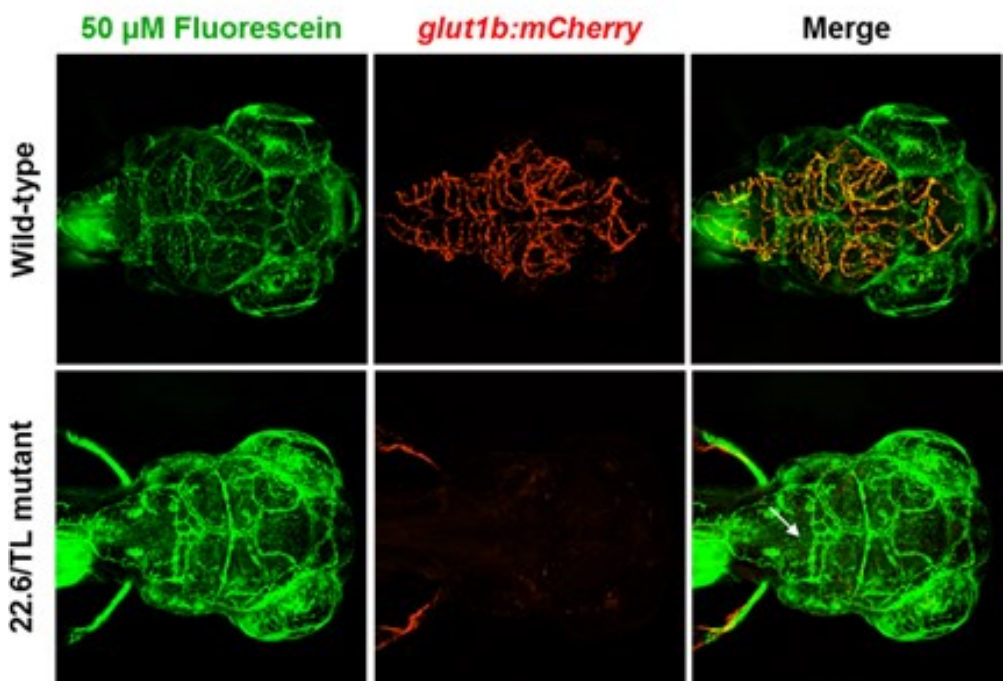

Figure 5-5. The 22.6/TL mutant blood-brain barrier is leaky with various sized tracers

4 dpf wild-type siblings and 22.6/TL mutants were either injected with $1 \mathrm{nl} 10 \mathrm{kDa}$ Rhodamine (A) or bathed for 30 minutes in $50 \mu \mathrm{M}$ Fluorescein (B). Confocal imaging revealed that mutants have more leakage of each tracer into the brain (A arrowhead; B arrow). Even though Rhodamine gets into the brain parenchyma of 22.6/TL, it appears as if the blood-CSF barrier is still intact (A arrows). 


\section{CHAPTER 6. A CHEMICAL SCREEN TO IDENTIFY MODULATORS OF BLOOD- BRAIN BARRIER DEVELPOMENT}

\section{Introduction}

\section{Importance and Classification of Chemical Screens}

The development of new therapeutics has large cost and time implications in the management of health issues. It is estimated that developing a new drug takes 12-15 years with over $\$ 1$ billion in costs ${ }^{140}$. It is also important to sufficiently validate targets during lead drug discovery as this is one reason why drugs fail to work in the clinic ${ }^{140}$. With an advanced understanding in disease etiology and possibilities for therapeutic targets, small molecule discovery is an extremely prevalent avenue for the development of new drugs. Small molecule screens, which are more cost-effective and faster assays, haven been known to make a big impact on drug discovery such as in the field of cancer biology $^{141}$.

While it is imperative to advance the field of medicine with new compounds because of issues like antibiotic and chemotherapeutic resistance, small molecule screens may effectively repurpose drugs. This type of approach referred to as a "connection screen" takes well-characterized drugs and identifies compounds that regulate a biological process of interest ${ }^{142}$. Other kinds of screens include targeted, phenotypic, disease modifier, and library annotation. ${ }^{142,143}$ Targeted screens utilize genetic reporters and readout such as fluorescence is monitored. Secondary assays and follow-up with dose response curves are essential in showing the effectiveness of a chemical hit from a targeted screen ${ }^{143}$. Phenotypic screens are assays without any previous knowledge of the target, but many unrelated biological factors can go into affecting the phenotype ${ }^{143}$. One of the drawbacks in a phenotypic screen is the lack of known mechanism. Because compounds may not be extremely selective, a phenotype may not be caused by a small molecule binding to a suggested target ${ }^{143}$. However, many drugs have been successfully developed by phenotypic screens ${ }^{57}$. As a kind of genetic model, disease models may be created in order to find small molecules that target a pathway of interest. Disease modifier screens test small molecule libraries in order to try and rescue or reduce the progression of particular disease phenotypes. Lastly, library annotation screens take novel compounds and characterize them based on the phenotype that is produced in the screen assay. These screens help characterize newly synthesized compounds that may be very functionally diverse ${ }^{142}$. Regardless of the kind of screen, each of these can be performed in the zebrafish model depending on the hypothesis that needs to be tested.

\section{Chemical Screens in Zebrafish}

Zebrafish are an ideal organism for small molecule screens as they are a costeffective model, develop rapidly outside of the mother, are translucent in early 
development, high clutch numbers can be timed when generated, and are small enough to be used in a high- content and throughput fashion. If later stages are to be analyzed, pigmentation can also be prevented by treating with the chemical $\mathrm{PTU}^{142}$. Individual embryos can be placed in 96 and even 384 well plates and the embryos will still develop normally over time (Figure 6-1). Embryos and larvae can be staged prior to the developmental window that is desired for testing. Zebrafish can then be delivered to wells, embryo water may be carefully removed, and the desirable amount of drugging water can be added back or fish may be delivered to plates in a pre-designated volume. Drugs can then be added to the zebrafish after the pre-designated volume of water is added so as to result in a particular final concentration of drug. Depending on the stage that is to be screened, zebrafish incubate in the compounds for a period of time until they are phenotypically observed.

Small molecule screens can also supplement genetic screens which are commonly performed in zebrafish. Hits may modulate paralogs in the zebrafish genome that resulted from the genome duplication ${ }^{46,142}$. Transgenesis is also a common technique applied to the zebrafish which can easily be used in targeted screens. For example, the commonly used vascular reporter, $\operatorname{Tg}(f l i l a: E G F P)$ was used in a small molecule screen to identify compounds that effect development of the vertebrate retina ${ }^{144}$. Cells extracted from transgenic zebrafish may also be cultured in vitro and used to screen a higher volume of compounds ${ }^{145}$. However, the advantage of using the zebrafish is that behavior and physiological development of a vertebrate can be monitored versus the fewer and broader phenotypes assayed in in vitro systems. Absorption, distribution, metabolism, and excretion studies have not been established in zebrafish yet, but hydrophobicity as measured by $\log \mathrm{P}$ values can predict bioavailability in zebrafish treated with compounds with various molecular weights ${ }^{146}$. Zebrafish have been a successful model to repurpose drugs and studies have validated zebrafish small molecule screen hits in mice as well ${ }^{147}$.

\section{Materials and Methods}

\section{Chemical Libraries Tested}

Because of its availability in our department and clinical significance, the Food and Drug Administration (FDA) library was the first library we tested. Subsequent screens were done with the ENZO Wnt, Autophagy, and Neurotransmitter libraries. The ENZO libraries were tested with the help of Dr. Steven Finckbeiner.

\section{Staging, Dosing, and Screening Strategy}

For the FDA library screen, zebrafish matings were set-up between $T g$ (glut 1b:mCherry; flila:EGFP) males and TL females two days prior to drugging. Matings were timed so that embryos could be staged the next day. After the plates were void of unfertilized embryos, embryos were rinsed into a $1 \%$ Dimethyl sulfoxide 

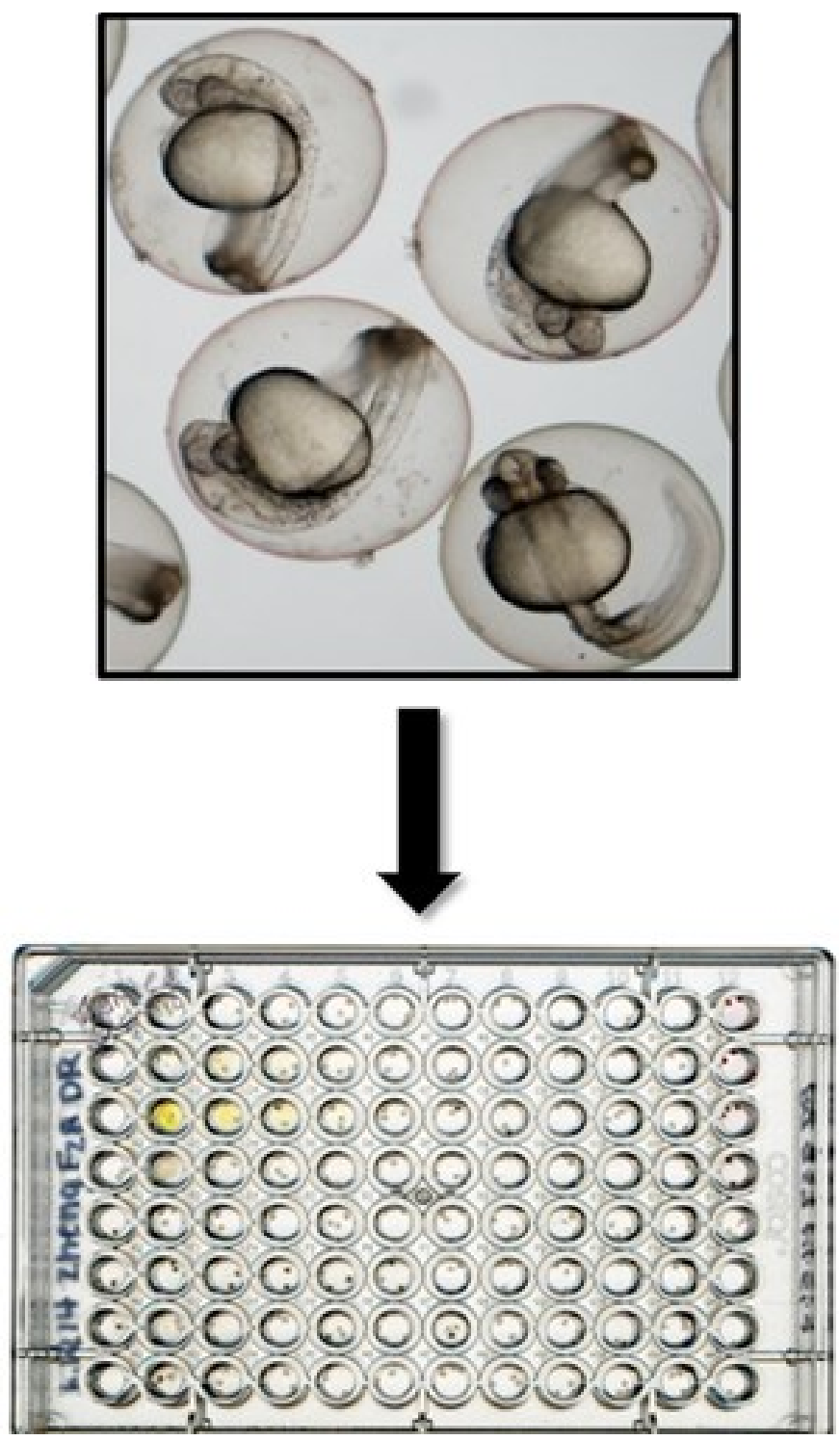

Figure 6-1. Zebrafish embryos are amenable for high-content small molecule screening

Multiple zebrafish embryos can be staged and easily placed into 96-well plates for chemical screens. 
(DMSO) solution. Three embryos per well were plated in the 1\% DMSO water onto a Perkin Elmer Cell Carrier black plate under the aid of a Nikon SMZ 1500 stereoscope. On drugging day, wells were checked for unfertilized eggs, water was removed, and 90 $\mu \mathrm{L}$ of $1 \%$ DMSO, PTU egg water was added to the wells. We used PTU water to prevent pigmentation in the animals and make fluorescence screening at $3 \mathrm{dpf}$ easier. An initial screening dose of $10 \mu \mathrm{M}$ was used in the FDA screen as that is a reasonable concentration in zebrafish small molecule screens ${ }^{142}$. $10 \mu \mathrm{L}$ of compound was transferred with a multichannel pipette from a 384 well drug library plate to the $90 \mu \mathrm{L}$ in the 96 well plate containing the $24 \mathrm{hpf}$ zebrafish embryos. This produced a 1:100 dilution from a $100 \mu \mathrm{M}$ stock plate, resulting in the final dosing concentration of $10 \mu \mathrm{M}$. Column 1 was always a negative control, containing fish in 1\% DMSO water only. Column 7 was always a positive control, containing $2 \mu \mathrm{M}$ Tunicamycin, a drug that we previously found caused brain hemorrhage and reduced BEC development. After drugging, plates were placed in a humidity chamber which consisted of moistened paper towels in a sandwich box. This box containing the drugged embryos was placed in the $28.5^{\circ} \mathrm{C}$ incubator. The incubator was on a 10 hour light 14 hour dark cycle like the zebrafish facility. Wells were monitored at $2 \mathrm{dpf}$ under brightfield to look for any abnormal development such as CNS necrosis or brain hemorrhage. At 3 dpf, embryos were assessed under brightfield optics as well as mCherry and GFP on a Nikon SMZ 1500 stereoscope with a PRIOR Scientific Lumen 200 PRO Fluorescence Illumination System. To score for changes in BBB development, embryos were anesthetized with $0.04 \%$ tricaine, around $300 \mu \mathrm{L}$ of cooled $1.2 \%$ low-melting point agarose was added to each well, the agarose hardened, and then plates were flipped upside down so that larvae were now stationary at the bottom of the 96 well plate for screening. Images were also recorded on the GE InCell 6000 in the High-throughput Screening core in the Chemical Biology and Therapeutics Department.

For the ENZO library screens, matings were set up like they were for the FDA screen. At one point, we switched to using $\operatorname{Tg}$ (glut 1:mCherry) males by $\operatorname{Tg}($ flila:EGFP) females to try and increase clutch size and the number of crosses we could set up. This time, embryos were cleaned but not plated until drugging at $24 \mathrm{hpf}$. For plating, embryos were rinsed from egg water into PTU and then into $1 \%$ DMSO water made in PTU. We also tried a range of doses for this library consisting of a 100,10, and $1 \mu \mathrm{M}$ dose for each compound plate. 3 embryos were transferred with either 198 or $180 \mu \mathrm{L} 1 \%$ DMSO, PTU water into the 96 well plates. The range of doses was carried out in a serial dilution on the Tecan Freedom Evo in the Chemical Biology and Therapeutics Department. Embryos in plate one had $198 \mu \mathrm{L}$ of water and $2 \mu \mathrm{L}$ of stock compound added for a final concentration of $100 \mu \mathrm{M}$. $20 \mu \mathrm{L}$ from this $100 \mu \mathrm{M}$ plate was transferred by the Tecan to plate two with $180 \mu \mathrm{L}$ to make a $10 \mu \mathrm{M}$ plate. Another $20 \mu \mathrm{L}$ from the $10 \mu \mathrm{M}$ plate was transferred by the Tecan to the third plate with $180 \mu \mathrm{L}$ to make the $1 \mu \mathrm{M}$ plate. Negative controls were in column 1, consisting of embryos in 1\% DMSO, PTU water only. Positive controls were in column 7 and consisted of 100, 10, or $1 \mu \mathrm{M} \mathrm{DMH} 4$, a potent angiogenesis inhibitor, or 20,2 , or $0.2 \mu \mathrm{M}$ Tunicamycin for plates one, two and three, respectively $^{105}$. Plates were scored on $2 \mathrm{dpf}$ and $3 \mathrm{dpf}$ just as they were for the FDA library screen (Figure 6-2). 


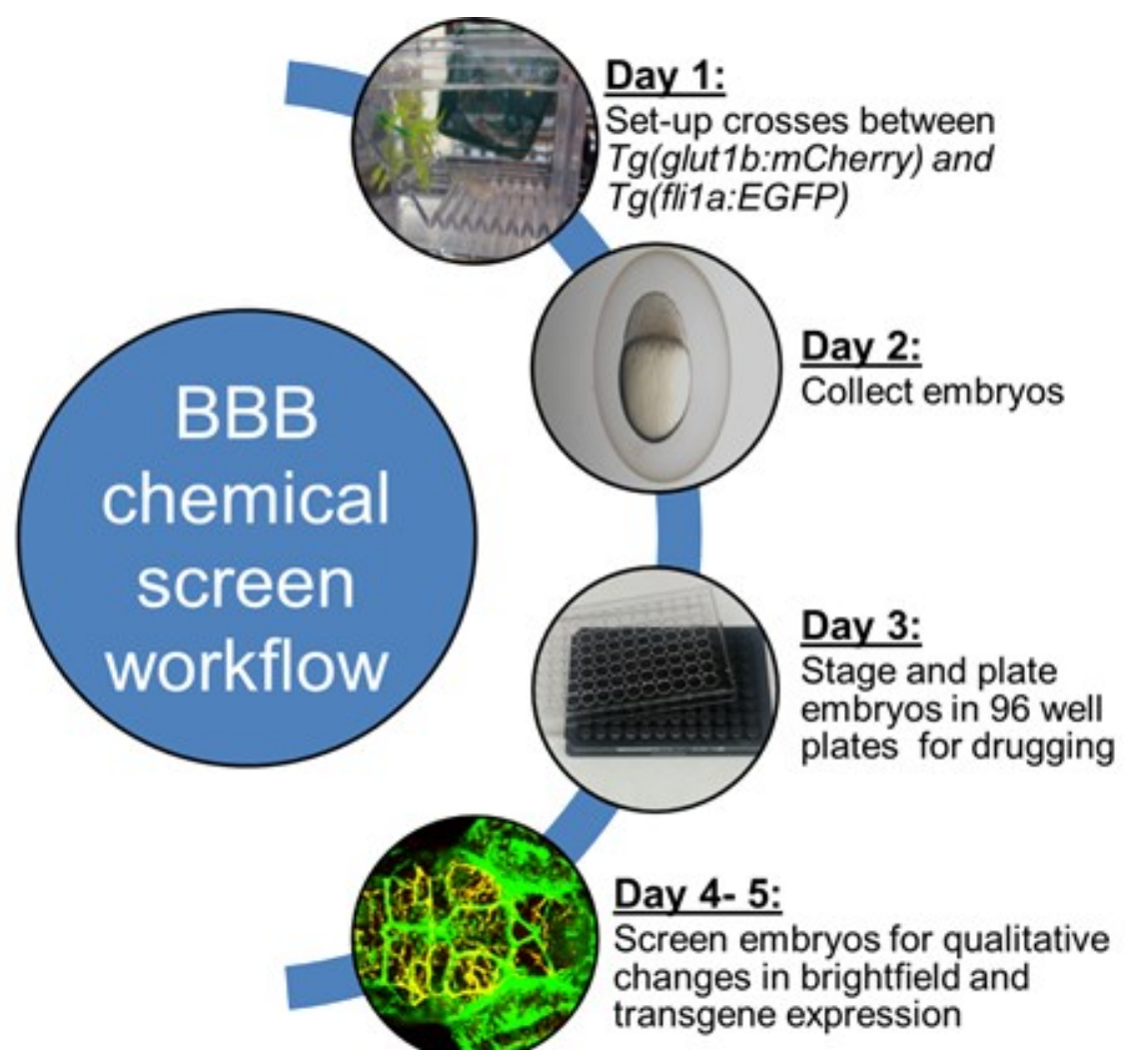

Figure 6-2. Weekly workflow for the blood-brain barrier chemical screen Zebrafish matings were timed between $T g$ (glutlb:mCherry;flila:EGFP) males and TL females or $T g$ (glut lb:mCherry) males and $T g(f l i l a: E G F P)$ females. Embryos were collected and rinsed the next day. On drugging day, embryos were staged at $24 \mathrm{hpf}$ in black bottom 96 well plates. Compounds were added to the 96 well plates already containing the zebrafish. At $2 \mathrm{dpf}$ embryos were scored qualitatively for developmental abnormalities and scored again at $3 \mathrm{dpf}$ for the same abnormalities as well as changes in reporter gene expression. 


\section{Statin Dose Response Testing}

Each of the 10 statins were tested for a dose response on $24 \mathrm{hpf}$ embryos. The range of doses went from $100 \mu \mathrm{M}$ down to $100 \mathrm{nM}$ decreasing stepwise in a factor of 3 . The dose responses for all 10 statins were repeated three times. Statins were acquired from Compound Management in the Chemical Biology and Therapeutics department. Statin stocks were in $100 \%$ DMSO and diluted into $1 \%$ DMSO, PTU egg water during treatment. Zebrafish were monitored for CNS necrosis, brain hemorrhage, and death at each dose from 24 to $48 \mathrm{hpf}$.

\section{Tunicamycin, Forskolin, and GBR13069 Treatment}

24 hpf Tg (glut1b:mCherry; flila: EGFP) embryos were dosed with $1 \mu \mathrm{M}$ Tunicamycin, which blocks N-linked glycosylation, 1- $10 \mu \mathrm{M}$ Forskolin, which elevates cyclic adenosine monophosphate (cAMP), and $100-6.25 \mu \mathrm{M}$ GBR13069, a dopamine uptake inhibitor. All compound stocks were in $100 \%$ DMSO and diluted in 1\% DMSO, PTU water for treatment. The GBR13069 dose response was performed by Dr. Steven Finckbeiner.

\section{Dolichol Kinase (dolk) Morpholino Injections and RT-PCR}

$1 \mathrm{nl}$ of a $0.5 \mathrm{mM}$ start site morpholino (TAAACATTGTCCACTTCTTGTCCTC) was injected into the cell of $\operatorname{Tg}($ glut 1 : $m$ Cherry; flila: EGFP) embryos at the single cell stage. Embryos were monitored over development and scored at $2 \mathrm{dpf}$ for hemorrhages. All microinjections were performed using a PV820 Pneumatic PicoPump (WPI). Injection volumes were calculated using a $0.01 \mathrm{~mm}$ Stage Micrometer (Fisher Scientific).

RT-PCR was performed on cDNA from 0-5 dpf zebrafish. RNA was extracted from 10 embryos per tube with 50 $\mu 1$ Trizol. Following Trizol extraction, RNA was recovered with a phenol chloroform spin, isopropanol and glycogen extraction, and $75 \%$ ethanol wash. cDNA was synthesized after the Trizol RNA extraction by using an Invitrogen SuperScript III First Strand kit. PCR was performed with dolk (forward, GTTGAATCTGCGGTGGTGTT and reverse, CAATCCCAAAACCGCAGTCA) and actin (forward, TGAATCCCAAAGCCAACAGAG and reverse, TCACACCATCACCAGAGTCC ) primers using the Invitrogen Accuprime Taq polymerase system and a Biorad C1000 Thermal Cycler.

\section{Microscopy}

After treatment or injection, larvae were observed and stage selected for microscopic documentation. Fish were anesthetized with $0.04 \%$ tricaine and then embedded dorsal side down in $1.2 \%$ low-melting point agarose in a glass coverslip 
bottom petri dish. Confocal images were acquired together for brightfield, mCherry and GFP on the Nikon C1Si. Brightfield z-stack images were acquired on the Nikon AZ100.

\section{Results and Discussion}

\section{FDA Library Chemical Screen Hit: Statins}

From the FDA library, six 384 well plates were tested totaling 884 compounds. The library was added to $24 \mathrm{hpf} T g$ (glut 1b: mCherry; flila: EGFP) embryos to bypass any effects on initial development but to still target development prior to CNS angiogenesis at $30 \mathrm{hpf}$. Our most penetrant hit was a class of compounds known as statins. Statins are commonly given to patients for lipid lowering therapy to help prevent coronary heart disease and are well developed inhibitors of the rate-limiting enzyme in cholesterol biosynthesis, HMG-CoA reductase ${ }^{148}$. Rosuvastatin, a commonly prescribed statin, was actually found as an anti-angiogenic inhibitor in another zebrafish chemical screen, but compounds in that study were added at $20 \mathrm{hpf}$ before intersegmental vessels formed in the embryo ${ }^{60}$. Outside of early development, statins are also known to have beneficial side effects in neurological disorder therapy via pleiotropic effects on downstream isoprenoid synthesis ${ }^{149}$.

To our surprise, we found that depending on the statin, embryos developed brain hemorrhage and/or CNS necrosis at $2 \mathrm{dpf}$ and even death after 24 hours of exposure (Figure 6-3). Dose responses were repeated three times for each statin and a heat map was generated to depict the half maximal effective concentration $\left(\mathrm{EC}_{50}\right)$ for each statin

(Figure 6-4). As depicted in this heat map for a selection of the statins tested, more lipophilic compounds such as Simvastatin caused severe side effects at lower $\mathrm{EC}_{50} \mathrm{~S}$ compared to less lipophilic statins like pravastatin. In fact, hemorrhage was seen as high as $30 \mu \mathrm{M}$ pravastatin but as low as $100 \mathrm{nM}$ with simvastatin. Pravastatin was not originally a hit in our FDA screen because the $10 \mu \mathrm{M}$ dose we used for screening was too low to cause hemorrhage in the zebrafish. This result is congruent with the BBB literature, reiterating that lipophilicity is one of the drug qualities that affect BBB penetration ${ }^{5}$. Statins also vary at the pharmacokinetic level when it comes to bioavailability and cytochrome p450 drug metabolism, suggesting another mechanism for the different effects among this class of drug ${ }^{148}$.

We then wanted to know if CNS angiogenesis would only be affected if treatment was performed before or after 24 hpf. Our lab and others have shown that the BBB develops early in the zebrafish, but we wanted to know if statins still have an effect on CNS angiogenesis in a zebrafish after the initiation of barriergenesis ${ }^{62}$. To answer this question, a time course with $1 \mu \mathrm{M}$ Atorvastatin and $10 \mu \mathrm{M}$ Rosuvastatin was performed as outlined in (Figure 6-5). For treatments done before $4 \mathrm{hpf}$ prior to zygotic transcription, larvae at $2 \mathrm{dpf}$ had a reduction in CNS angiogenesis (Figure 6-6, top panel). Interestingly enough as seen with a well-known inhibitor of angiogenesis, statins 


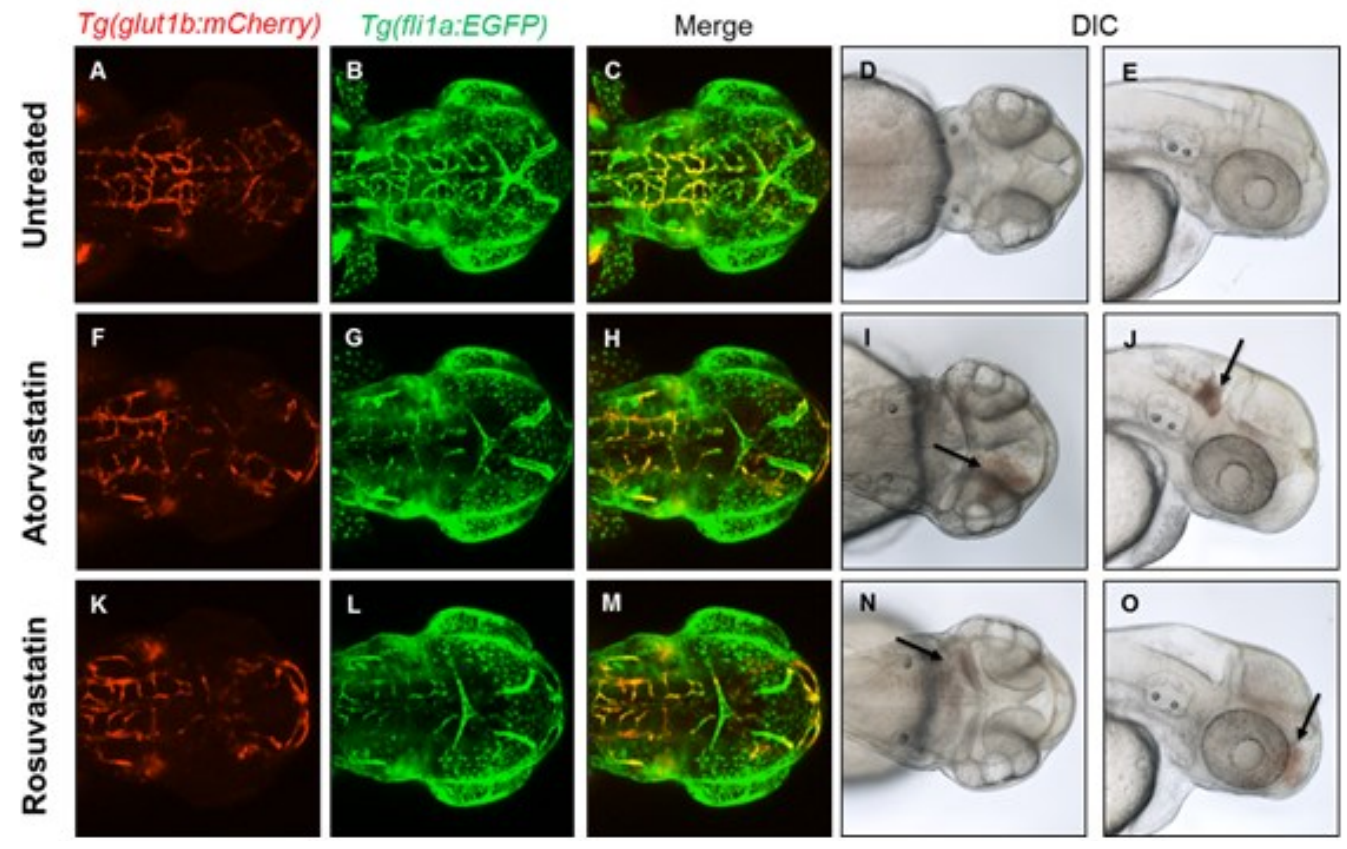

Figure 6-3. Statins induce cerebral hemorrhage and reduce CNS angiogenesis in the developing zebrafish

Compared to untreated siblings (B), Tg (glut1b:mCherry; flila:EGFP) embryos treated at $24 \mathrm{hpf}$ with $1 \mu \mathrm{M}$ Atorvastatin $(\mathbf{G})$ or $10 \mu \mathrm{M}$ Rosuvastatin $(\mathbf{L})$ had reduced CNS angiogenesis. Levels of mCherry expression in statin treated embryos (F, K) were still similar to control (A). Statin treated embryos also developed cerebral hemorrhages as seen in dorsal views (I, $\mathbf{N}$ arrows) and side views (J, $\mathbf{O}$ arrows) unlike their untreated siblings (D, E). 


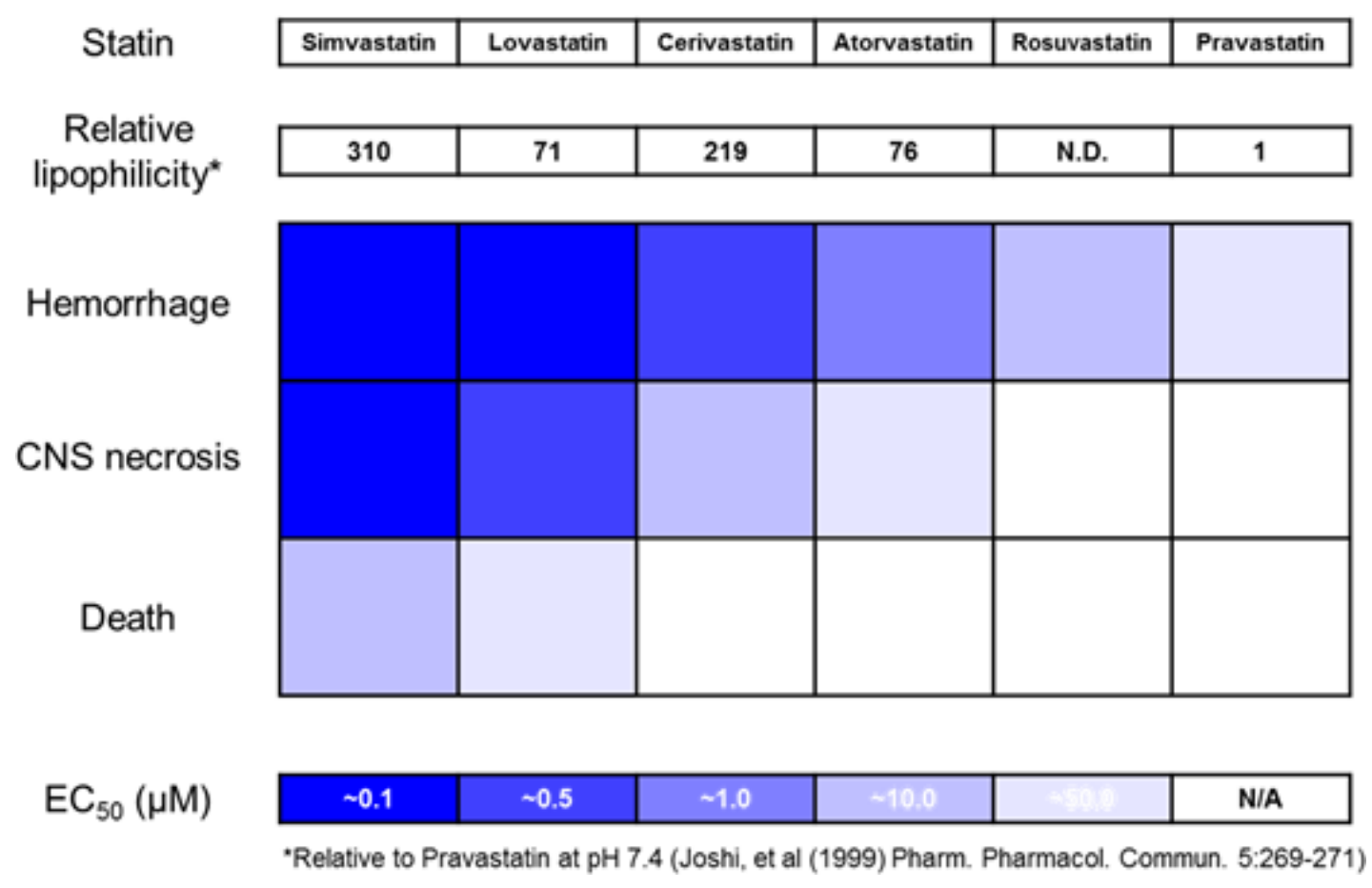

Figure 6-4. Zebrafish as a predictive indicator of blood-brain barrier penetration of statins

Depending on the lipophilicity of the statin, zebrafish developed an array of phenotypes ranging from brain hemorrhage to death. More lipophilic statins like simvastatin cause more severe side effects at lower doses versus a less lipophilic statin like pravastatin. 


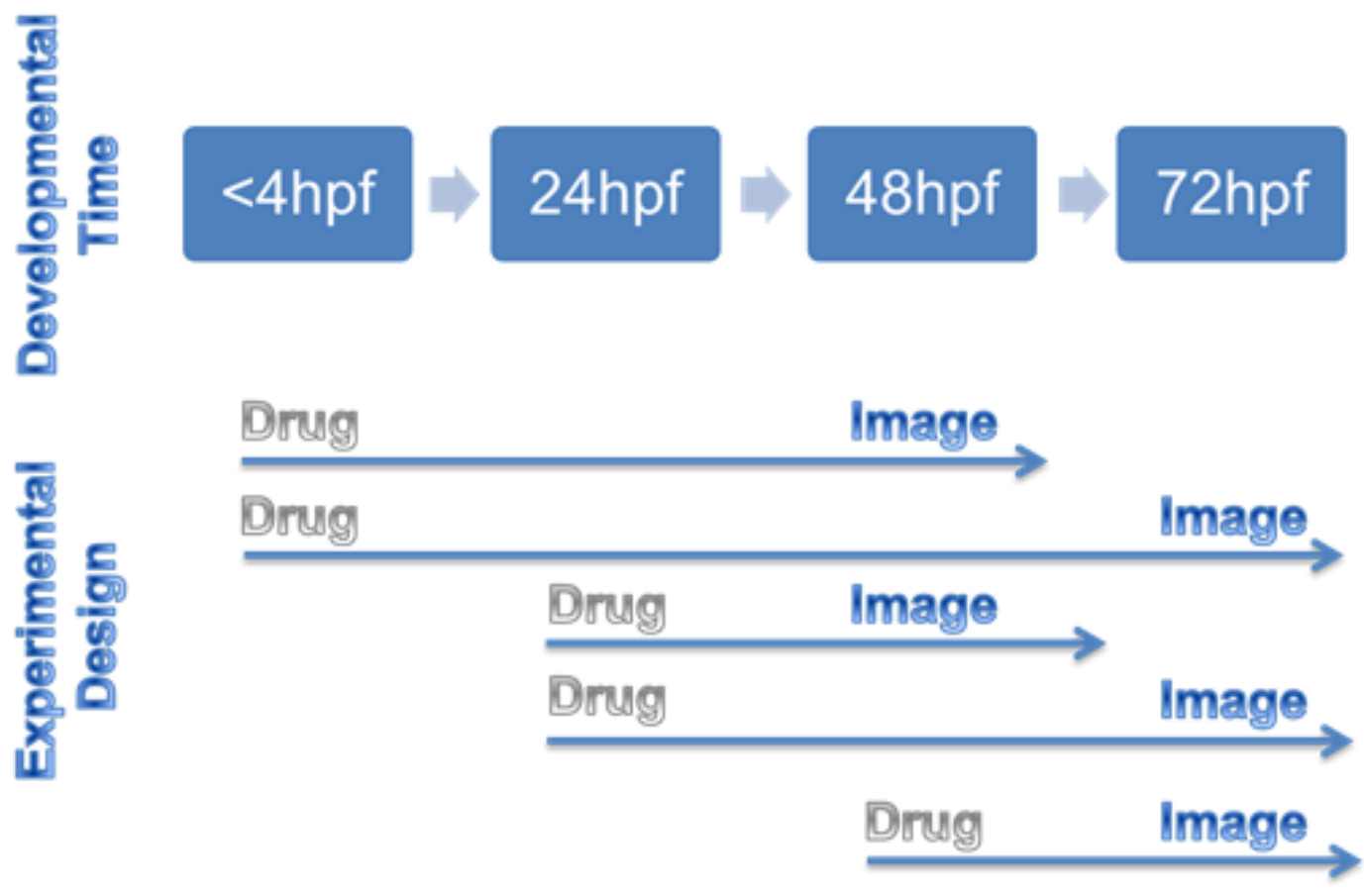

Figure 6-5. Experimental design for a developmental time course with Atorvastatin and Rosuvastatin treatments

Tg (glutlb:mCherry; flila:EGFP) embryos were treated with $1 \mu \mathrm{M}$ Atorvastatin or 10 $\mu \mathrm{M}$ Rosuvastatin and then imaged throughout the course of development to see if the effects of statins still persisted before or after the initiation of barriergenesis. 


\section{4hpf treatment, $48 \mathrm{hpf}$ images}

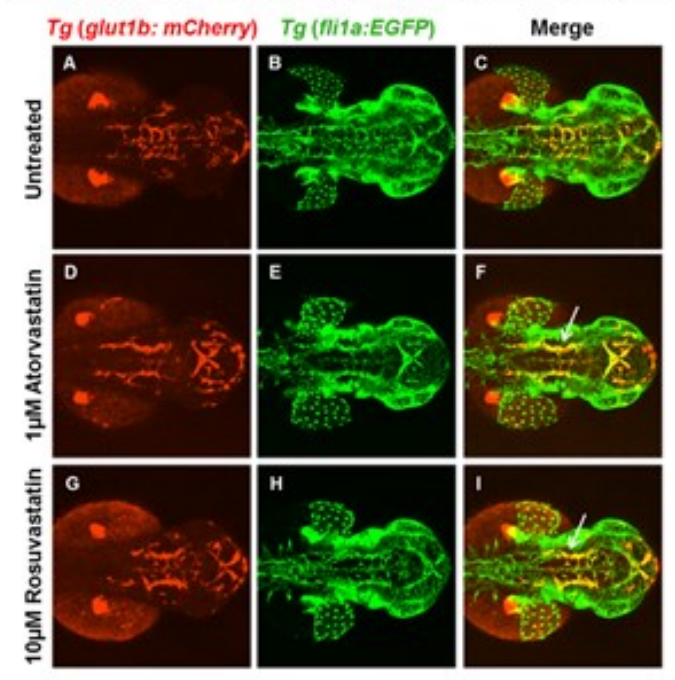

\section{4hpf treatment, 72hpf images}

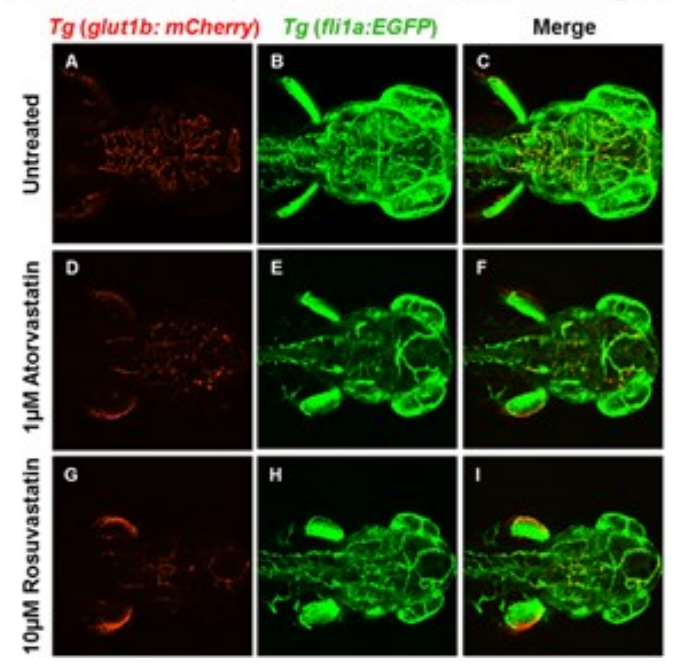

Figure 6-6. Statin treatment prior to zygotic transcription disrupts CNS angiogenesis

Tg (glutlb:mCherry; flila:EGFP) embryos were treated with $1 \mu \mathrm{M}$ Atorvastatin or 10 $\mu \mathrm{M}$ Rosuvastatin before $4 \mathrm{hpf}$. The top panel shows that by $2 \mathrm{dpf}, \mathrm{CNS}$ angiogenesis is disrupted in statin treated fish $(\mathbf{E}, \mathbf{H})$ compared to untreated siblings (B). Interestingly, the PHBCs of statin treated embryos still undergo barriergenesis as indicated by glut $1 \mathrm{~b}$ expression (F, I arrows) even though CNS angiogenesis is abnormal. The lower panel shows that by $3 \mathrm{dpf}$, larvae have an even more severe reduction in CNS angiogenesis after incubating in statins for a longer period of time. Treated embryos also seem to express glut $1 b$ ectopically in the brain $(\mathbf{D}, \mathbf{G})$. 
delivered less than $4 \mathrm{hpf}$ prevent CNS angiogenesis but still allow glut lb positive PHBC development to occur (Figure 6-6F and I, top panel arrows). Embryos that incubated in statins for another $24 \mathrm{~h}$ until $3 \mathrm{dpf}$ had a more severe effect on brain vasculature development and glut $1 b$ even seemed to be ectopically expressed (Figure 6-6D and G, bottom panel) For treatment performed at 24 or $48 \mathrm{hpf}$ until $72 \mathrm{hpf}$, statins still disrupted the development of the brain vasculature (Figure 6-7). While CNS angiogenesis was disrupted when treatment was done after 2 dpf (Figure 6-7E and $\mathbf{H}$, bottom panel), treatment performed at $24 \mathrm{hpf}$ until $72 \mathrm{hpf}$ had a more severe effect on the development of BECs (Figure 6-7E and $\mathbf{H}$, top panel). Treatment done later from $5 \mathrm{dpf}$ onward did not produce any brain hemorrhage in the developing larvae.

While a developmental time course of statin treatment had not been performed in the zebrafish, other groups have previously demonstrated brain hemorrhage induced by statins during the time we were performing our FDA screen ${ }^{150,151}$. These groups suggested hemorrhage was due to inhibition of protein prenylation and absence of Coenzyme Q10. While these hypotheses were validated, the cholesterol biosynthetic pathway affects another major downstream biochemical process, N-linked glycosylation. For our small molecule screens we had been using Tunicamycin, a well-known inhibitor of N-linked glycosylation, as a positive control. As seen after $1 \mu \mathrm{M}$ Tunicamycin treatment at $24 \mathrm{hpf}$, embryos developed brain hemorrhages and had abnormal CNS angiogenesis just like statin treated embryos (Figure 6-8). To try and draw a mechanistic connection between statin-related brain hemorrhage and abnormal vessel development, we decided to knock down dolk with morpholino technology. Dolk is an enzyme active at the endoplasmic reticulum, important for $\mathrm{N}$-linked glycosylation, and is upstream of Tunicamycin's target. By knocking down dolk, we could investigate whether our chemical treatment could be phenocopied genetically with morpholinos. While not $100 \%$ penetrant, $1 \mathrm{nl}$ of a $0.5 \mathrm{mM}$ dolk start site morpholino injection produced brain hemorrhage in $10-15 \%$ of morphants as well as abnormal CNS angiogenesis (Figure 6-9B, C). Hemorrhage was also seen at a higher dose of $1 \mathrm{nl}$ of $1 \mathrm{mM}$ morpholino. Morphants also developed a smaller, dented head, curved tails, heart edema, and had heartbeat without circulation. Because dolk is maternally derived (Figure 6-9A), this may contribute to the low penetrance of brain hemorrhage especially since the morpholino becomes more dilute over time. The diffusion of the morpholino may also explain why the reduction in CNS angiogenesis in dolk morphants was not as severe as chemical treatment was with Tunicamycin. Similar to statin treatment after $24 \mathrm{hpf}$, the dolk morpholino did not disrupt peripheral angiogenesis (Figure 6-10).

\section{ENZO Wnt and Autophagy Library Hit: Forskolin}

For the ENZO libraries, we started off with the Wnt and Autophagy compounds. Wnt is known to be important for barriergenesis as well as a regulator of Glut1 expression, so it was expected to find hits from this library ${ }^{36,102}$. To our surprise, Forskolin was a hit common to both the Wnt and Autophagy libraries. Forskolin is a well-known inhibitor of cAMP production. When dosed at $24 \mathrm{hpf}, 10 \mu \mathrm{M}$ Forskolin caused abnormal CNS angiogenesis, extreme kinking of the tail, and seizure-like 


\section{4hpf treatment, $72 \mathrm{hpf}$ images}

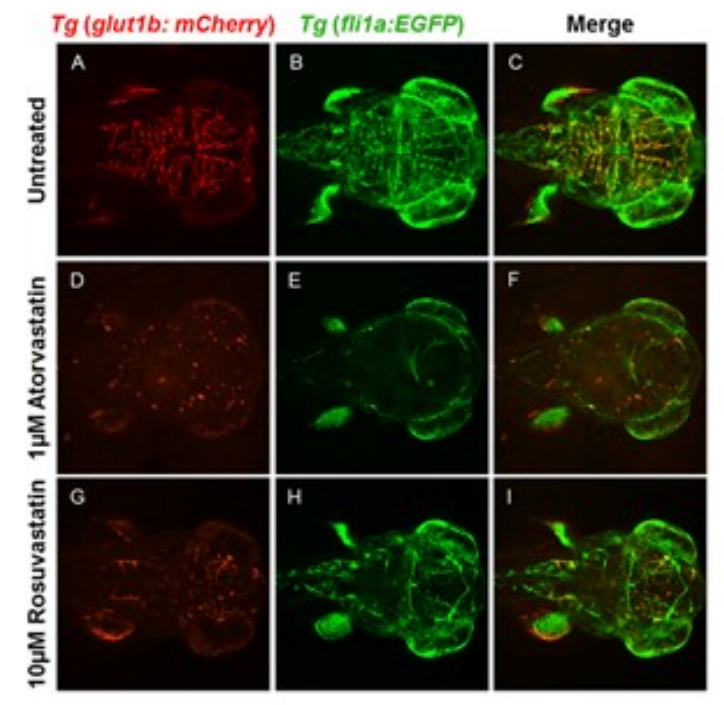

\section{8hpf treatment, $72 \mathrm{hpf}$ images}

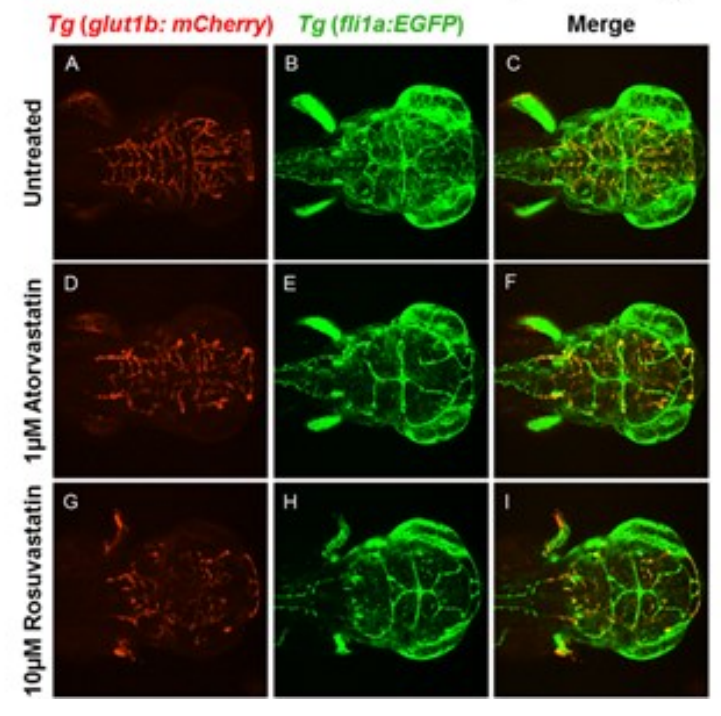

Figure 6-7. Statin treatment from 24 or 48 hpf to 72 hpf disrupts CNS angiogenesis

Tg (glutlb:mCherry; flila:EGFP) embryos were treated with $1 \mu \mathrm{M}$ Atorvastatin or 10 $\mu \mathrm{M}$ Rosuvastatin at 24 or $48 \mathrm{hpf}$ until $72 \mathrm{hpf}$. The top panel shows that by $3 \mathrm{dpf}, \mathrm{CNS}$ angiogenesis is disrupted in statin treated fish $(\mathbf{E}, \mathbf{H})$ compared to untreated siblings $(\mathbf{B})$ when embryos incubate in compound for 2 days. The lower panel shows that later treatment at $48 \mathrm{hpf}$ still causes a reduction in CNS angiogenesis $(\mathbf{E}, \mathbf{H})$ albeit less severe than statin treatment at $24 \mathrm{hpf}$ for 48 hour exposure. 
A
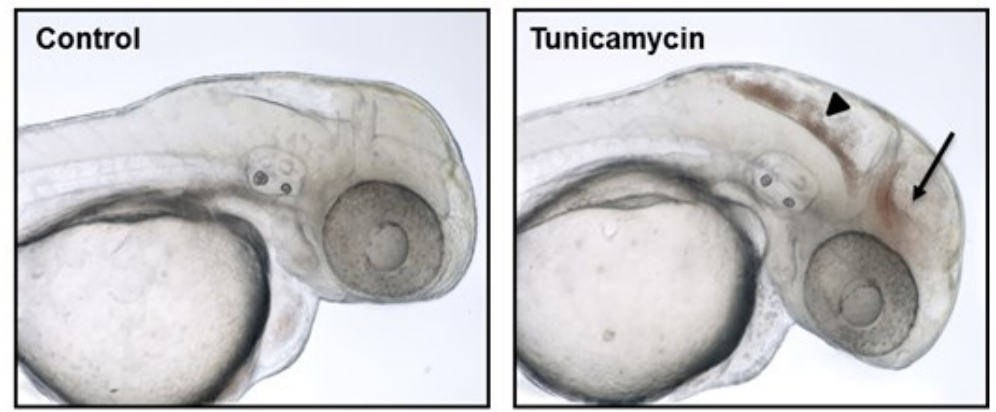

B
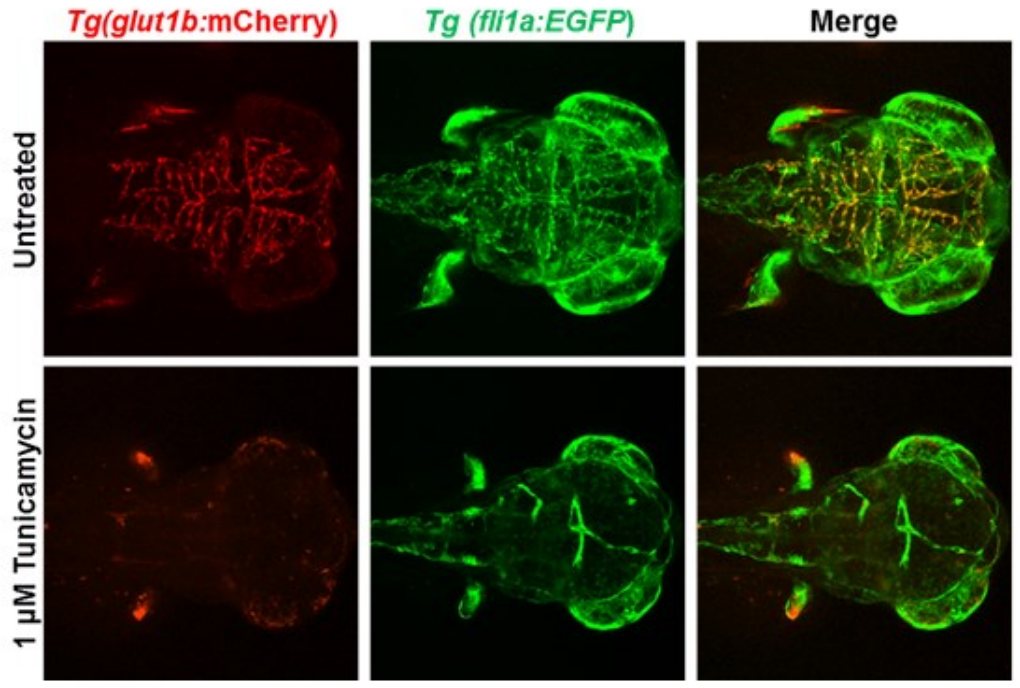

Figure 6-8. Tunicamycin causes a similar disruption to CNS vessels as does statin treatment

Tg (glut1b:mCherry; fli la:EGFP) embryos were treated with1 $\mu \mathrm{M}$ Tunicamycin at 24 hpf. The next day after treatment, embryos developed hemorrhages in the brain (A, arrow) and ventricle (A, arrow head) similar to what was seen with statin treatment. Confocal microscopy revealed that CNS angiogenesis was also disrupted in Tunicamycin treated embryos $(\mathbf{B})$. 
A

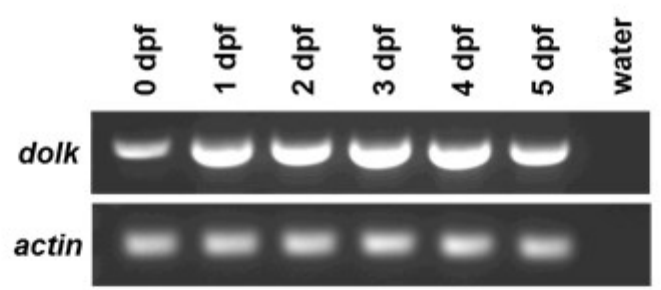

B
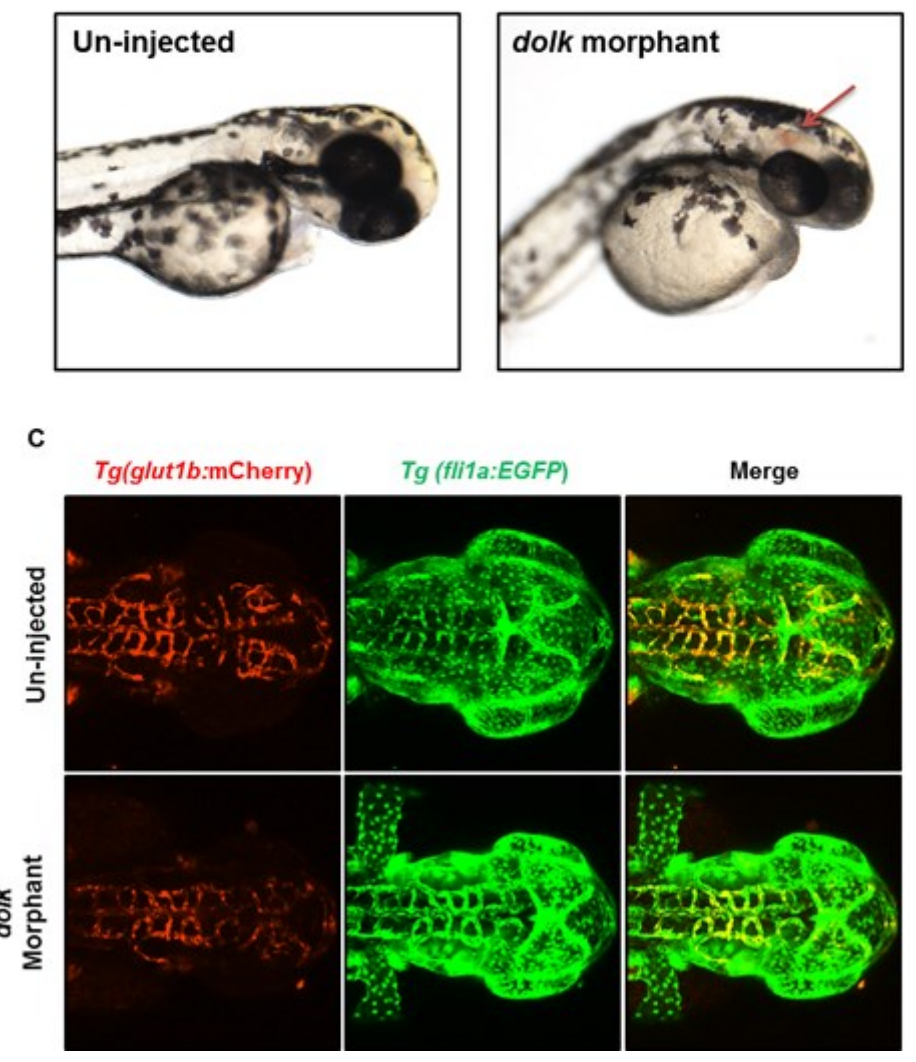

Figure 6-9. dolk is maternally derived and disrupts CNS angiogenesis when targeted with morpholinos

RT-PCR was performed for the dolk transcript on $0-5$ dpf cDNA (A). $1 \mathrm{nl}$ of a $0.5 \mathrm{mM}$ dolk start site morpholino caused $10-15 \%$ of morphants to develop brain hemorrhages at $2 \mathrm{dpf}$ (B). Confocal microscopy revealed abnormal CNS angiogenesis in morphants compared to un-injected siblings (C). 


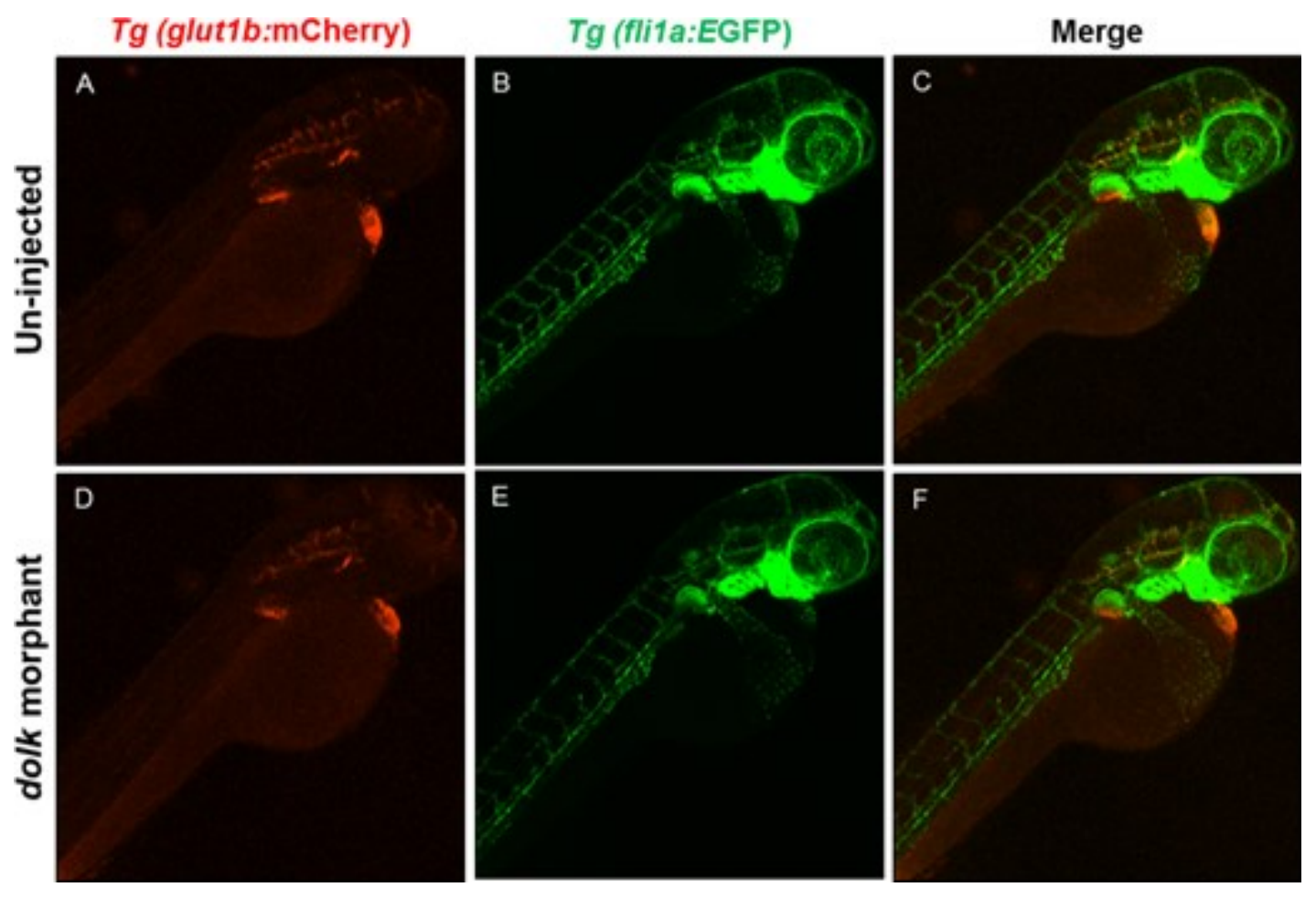

Figure 6-10. dolk morphants have normal peripheral angiogenesis

Knockdown of dolk in Tg(glut1b:mCherry; flila:EGFP) embryos does not affect the development of peripheral endothelium (E ) compared to the development in un-injected control siblings (B). 
behavior (Figure 6-11). It is not known whether the spastic behavior of the Forskolin treated fish was neuro-muscular or seizure-induced but it was a very pronounced phenotype. A dose response with Forskolin also showed a dose-dependent phenotype for this compound (Figure 6-11B).

\section{ENZO Neurotransmitter Library Hit: GBR13069}

The BBB maintains critical interactions with the cells of the NVU. Groups have investigated the role of astrocytes and pericytes, but not a lot has been published on the contribution of neurons to barriergenesis ${ }^{27,33,41}$. To test the impact of NTs during BBB development, agonists and antagonists of common NTs were added to developing $T g$ (glut 1b:mCherry; flila:EGFP) embryos at $24 \mathrm{hpf}$. For this library, we also decided to test a range of doses, as it has been demonstrated that convulsant agents like Pentylenetetrazole work in the $\mathrm{mM}$ range in zebrafish ${ }^{152}$. Our most pronounced hit was a dopamine transporter agonist called GBR13069. In vitro and in vivo experiments with GBR13069 have validated it as a potent dopamine uptake inhibitor, showing increased motor availability in naïve mice ${ }^{153}$. When screening on $3 \mathrm{dpf}$, we noticed that embryos treated with $100 \mu \mathrm{M}$ had a large reduction in mCherry expression. After a subsequent dose response with GBR13069 and confocal image acquisition, we noticed a reduction in mCherry expression specifically in the hindbrain of treated embryos (Figure 6-12). This regional specificity is not surprising as it has been demonstrated that different Wnt ligands are also expressed regionally and loss of Wnt signaling has a compartmental effect on the BBB in the forebrain and ventral spinal cord ${ }^{36}$. Localization of dopamine receptor in the zebrafish are also expressed in the hindbrain rhombomeres, areas where the CtAs branch off of the PHBCs ${ }^{108,154}$.

\section{Conclusion}

We performed small molecule screens for chemical modulators of the BBB in our Tg (glutlb:mCherry; flila:EGFP) line with the FDA and select ENZO drug libraries. With the FDA library we found that our main hit, statins, caused cerebral hemorrhage, CNS necrosis, and even death when added to embryos at $24 \mathrm{hpf}$. We saw that when treated before zygotic transcription, larvae still had abnormal CNS angiogenesis but PHBCs underwent barriergenesis as indicated by glut $1 b$ expression. We have previously seen a similar result by inhibiting VEGF signaling in BECs, confirming that CNS angiogenesis and barriergenesis occur via independent mechanisms. When treated with statins before or after $24 \mathrm{hpf}$, larvae still had abnormal CNS angiogenesis and brain hemorrhage.

The severity of the phenotypes correlated to the lipophilicity of each statin, suggesting that zebrafish can be a predictive model for statin brain penetration. CNS brain hemorrhage in zebrafish after statin treatment has also been reported by other groups and helps validate this hit from our independent screen ${ }^{150,151}$. These groups suggest the brain hemorrhages were due to a modification in protein prenylation. This 


\section{A}
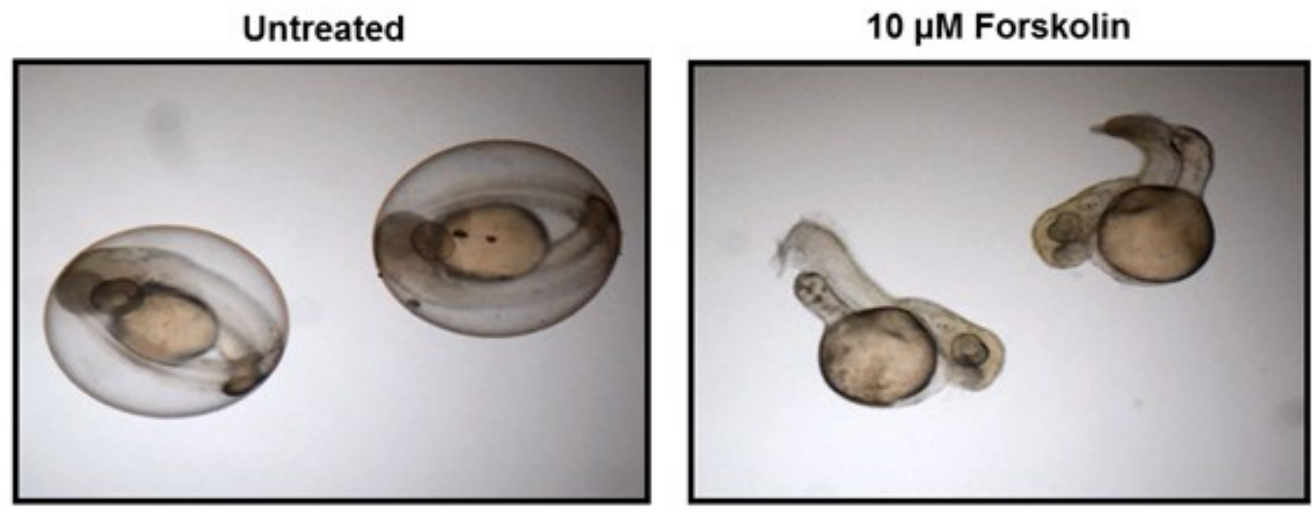

B
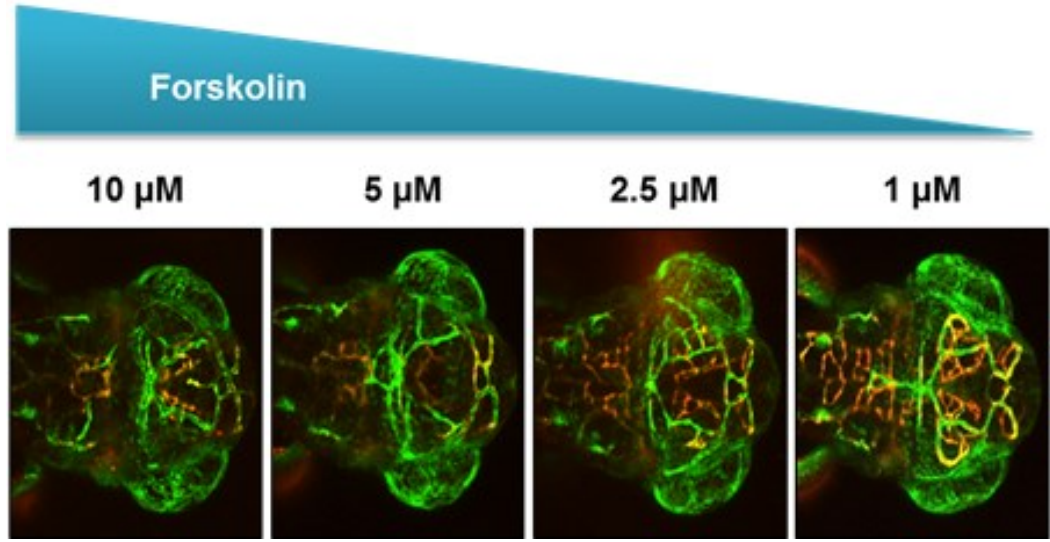

\section{Untreated}

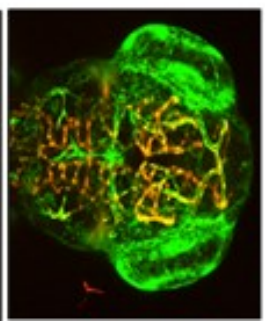

Figure 6-11. Forskolin was a hit from both ENZO Wnt and Autophagy libraries $10 \mu \mathrm{M}$ Forskolin treatment at $24 \mathrm{hpf}$ on Tg (glut 1b:mCherry; flila:EGFP) embryos caused developmental abnormalities and seizure-like activity by $48 \mathrm{hpf}(\mathbf{A})$. A dose response with Forskolin from 10 to $1 \mu \mathrm{M}$ shows a disruption in CNS angiogenesis (B). 


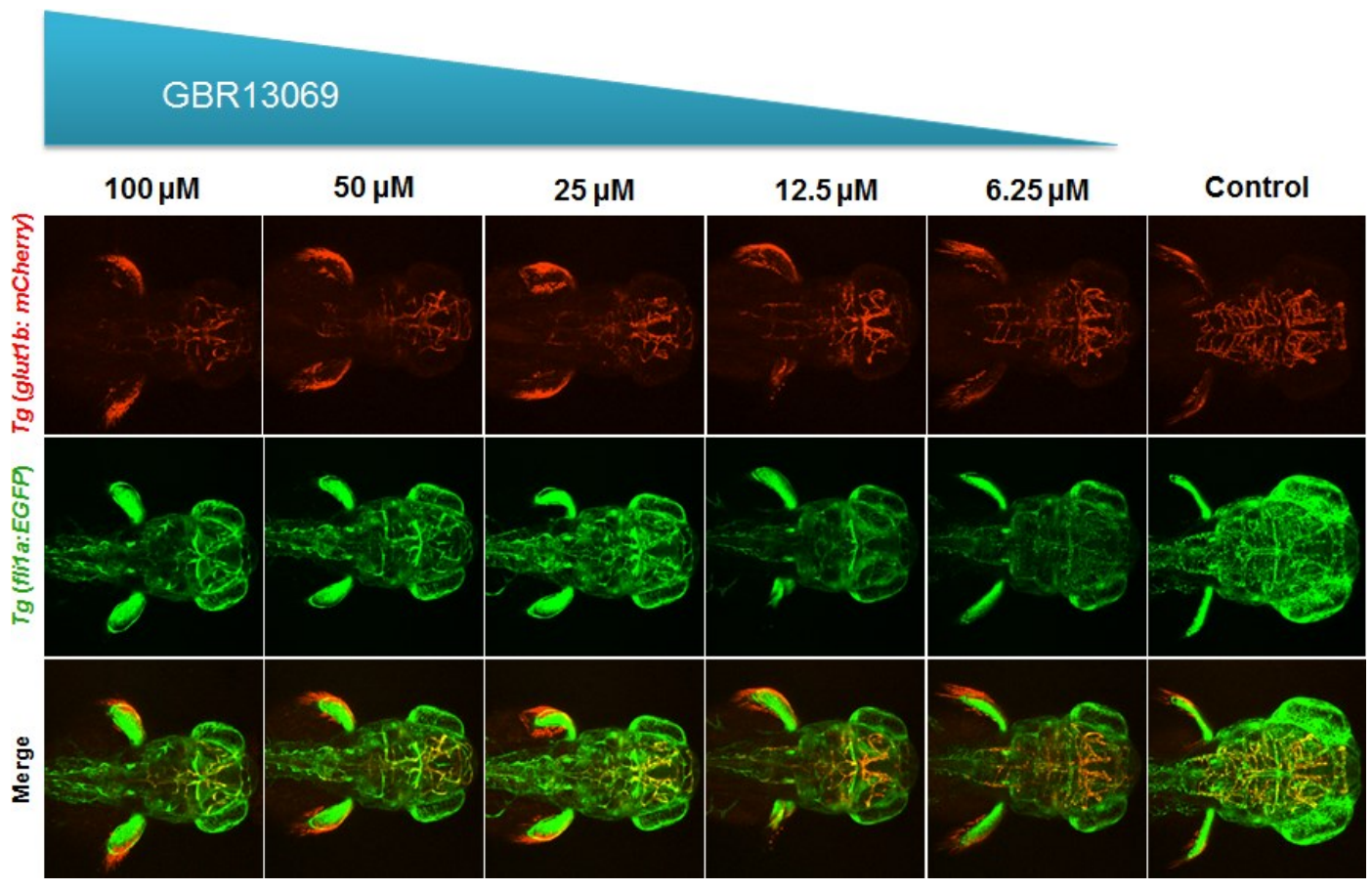

Figure 6-12. GBR13069 dose response shows a decrease in $\mathrm{Tg}$ (glut1b:mCherry) in the hindbrain of the developing zebrafish

GBR13069 affects the hindbrain vasculature when treated at $24 \mathrm{hpf}$. This effect regresses with decreasing doses of the compound. 
cellular process modifies many important signaling pathways such as Ras, laminin, $\mathrm{Rho} / \mathrm{Rac}$, and G-proteins and such signaling events have been implicated in BECs ${ }^{155,156}$. However, it is important to note that while these other groups suggest mechanisms through protein prenylation, the cholesterol biosynthesis pathway has other downstream effects such as N-linked glycosylation. The positive control used in our small molecule screens, Tunicamycin, is an effective blocker of N-linked glycosylation. Coincidentally when embryos are treated at $24 \mathrm{hpf}$ with Tunicamycin, they also develop a phenotype similar to statin treated embryos. After morpholino injections against dolk, an enzyme involved in N-linked glycosylation upstream of Tunicamycin, we found that there was a $10-15 \%$ occurrence of brain hemorrhage in morphants. While brain hemorrhage did not occur in all of the morphants, carriers of the p21-activated kinase $2 a$ mutation in the redhead zebrafish mutant also had hemorrhage occurrence less than $100 \%{ }^{124}$. This demonstrates that phenotypes such as brain hemorrhage may not be $100 \%$ penetrant even with germline mutations. While correlative, this suggests that $\mathrm{N}$-linked glycosylation could be affected by statins during CNS angiogenesis.

From the ENZO Wnt and Autophagy libraries we screened, we found that Forskolin was a hit common to both sets of compounds. Enhancing cAMP levels in vitro enhances tight junctions through TEER and reduced permeability in BECs ${ }^{157}$. cAMP has also been shown to elevate the induction of the tight junction Claudin5 in porcine $\mathrm{BECs}^{158}$. However, processes that affect the initiation of barriergenesis may differ from signaling as it happens after the barrier is established in adults. For example, Wnt signaling is important for $\mathrm{BBB}$ maturation but is reduced in adults ${ }^{102}$. In addition to Forskolin from the ENZO libraries, we found that GBR13069, a dopamine uptake inhibitor, affects hindbrain CNS angiogenesis in the developing zebrafish. This regional specificity coincides with transcript expression of dopamine receptors and hindbrain PHBC development ${ }^{108,154}$. 


\section{CHAPTER 7. SUMMARY AND CONCLUSIONS}

Among the three CNS barriers, the BBB is crucial for defense against circulating xenobiotics and endogenous ligands that would otherwise pose a threat to the $\mathrm{CNS}^{3}$. The BBB is a complex, physiological arrangement of brain endothelium and interacting astrocytic endfeet, neurons, pericytes, and microglia, all composing the $\mathrm{NVU}^{23}$. Cell non-autonomous signals between these cells help the BBB adapt its physiological functions $\mathrm{s}^{31,33,35,36,40,41}$. However, the protection the BBB provides the CNS simultaneously confers drug resistance against therapeutics. These multi-drug resistant mechanisms hinder the treatment of CNS diseases because efficacious drug volumes cannot be delivered into the brain ${ }^{5}$. The paracellular barrier in between brain endothelium also prohibits the passive diffusion of compounds into the $\mathrm{CNS}^{2}$. On the contrary, many CNS syndromes such as Multiple Sclerosis, Alzheimer's and Parkinson's disease present alterations in the BBB and consequently augment disease advancement ${ }^{92}$. Gaining a better understanding of healthy brain barrier signaling and physiology could therefore benefit the targeted delivery of CNS therapies and impede the progression of CNS diseases.

The BBB has been studied for over a century, but only recently have molecular cues important for barrier development become uncovered ${ }^{31,33,35,40,64}$. While informative to the field, these signals have been discovered with a gene candidate approach. Finding novel genes that regulate the BBB in an unbiased methodology could advance the field of brain barrier biology.

We hypothesized that zebrafish could be used a model to dissect the molecular mechanisms important for BBB development, maintenance, and function. We proposed zebrafish could be used for unbiased chemical and genetic screening assays. To do this, we initiated two specific scientific aims. The first aim was to design a transgenic BBB reporter line as no specific BBB reporter lines have been published to date. For the second aim, we proposed to use our transgenic line for small-scale forward genetic and small molecule screens to discover molecular modulators of barriergenesis.

Zebrafish are genetically tractable vertebrates and their quick development time and transparent nature makes them very amenable for developmental studies. Zebrafish have been widely used for studying different biological processes and have identified various small molecule hits and genes involved in signaling pathways and physiological structures $^{57,109,115,146}$. The ease of mapping zebrafish genetic mutants have also made zebrafish an attractive model for genetic studies and the recent advancement in sequencing technology has promoted the appeal of using zebrafish for genetic screens ${ }^{128,136}$. Furthermore, zebrafish have recently been used to study brain barriers, showing they possess a functional and homologous barrier to mammals early in development ${ }^{61,62,66,76}$.

To achieve our first aim of creating a BBB transgenic reporter line, we identified GLUT1 as a major molecular marker for barriergenesis. Rodent BBB studies report 
GLUT1 as a functional indicator of barrier formation and a study simultaneously performed during the transgenesis of our model confirmed the specificity of Glut 1 in the zebrafish $\mathrm{BBB}^{27,28,36,64}$. To create our BBB transgenic line, we utilized the common Tol2kit to engineer a DNA construct with a $2.9 \mathrm{~kb}$ fragment of the glut $1 b$ promoter upstream of the mCherry transgene ${ }^{84}$. After engineering our BBB DNA construct, we micro-injected glut 1b:mCherry DNA and Tol2 transposase mRNA into single cell $\mathrm{Tg}$ (flila:EGFP) embryos. Observing transient expression, we identified larvae which expressed glut1b:mCherry specifically in flila:EGFP brain endothelium. After achieving germline transmission of our glut 1 : $m$ Cherry transgene, we saw expression colocalized only in brain vessels and not in the periphery or circumventricular organs which lack barrier properties. glut $1 b$ expression continued into adulthood and was also expressed in reproductive tissues.

One of the ultimate goals of creating the first BBB transgenic line was to visualize the initial stages of barrier development as that had not be done in real time. Two hypotheses exist, including that nascent CNS vessels are immature and "leaky" during the first stages of development or that they acquire barrier properties as soon as vessels migrate into the brain parenchyma ${ }^{1}$. By performing live confocal microscopy, we were able to visualize barriergenesis occurring simultaneously with CNS angiogenesis starting at $30 \mathrm{hpf}$. This was the first time the initiation of barriergenesis had been demonstrated in a live vertebrate. With the creation of our $T g$ (glut 1b:mCherry) transgenic line, we were therefore able to answer one of the paramount questions in brain barrier biology.

We then wanted to characterize our BBB transgenic line to provide additional evidence that the zebrafish BBB is comparable to mammals as well as tease a part signaling pathways in a model system amenable to such assays. To assess known molecular markers of the mammalian BBB in the zebrafish, we performed IHC on $T g$ (flila:EGFP) sections over the course of zebrafish development. We found that the transporters Glut1 and Mdr1 as well as the tight junction protein Claudin5 are expressed as early as $2 \mathrm{dpf}$ and continue their expression into zebrafish adulthood. We already demonstrated that one of these barrier properties, glut $1 b$, is expressed during initial CNS angiogenesis, but we wanted to know if these newly formed vessels were also functional. We performed IV injection of an Albumin tracer conjugated with an Alexa Fluorophore 647. Upon injection and tracer circulation, we were able to visualize that newly formed brain vessels were impermeable to Albumin. While these results indicate that the zebrafish BBB is functional and molecularly comparable to mammals, future studies could examine the histological presence of other markers such as ZO-1, organic anion transporters, and BCRP, as well as investigate if smaller tracers are more amenable to leak out of nascent BECs.

Zebrafish provide an excellent model organism for studying developmental signaling pathways and because of this, we wanted to tease a part Wnt signaling in the zebrafish $\mathrm{BBB}$ as it had been demonstrated to function in the mammalian $\mathrm{BBB}^{31,36}$. These other Wnt studies used conditional mouse knockouts which were embryonic lethal and therefore the BBB could not be studied past a particular time point. Even though various Wnt ligands are expressed in a spatial manner throughout the brain, this is not 
surprising as Wnt signaling is important to development of neural circuitry in mammals ${ }^{37}$. To circumvent developmental abnormalities that could affect barriergenesis before it started, we crossed our BBB transgenic line to an inducible heat shock transgenic line to express a negative regulator of Wnt signaling, Axin-1. By performing heat shock after 1 hour, we were able to demonstrate that CNS angiogenesis is ablated and barrier properties are ectopically expressed as they are in the mammal ${ }^{36}$. It is satisfying that we reproduced a result seen in mammals in our BBB line, but without BECs it is impossible to study barrier properties. To evade this issue, we titrated back the amount of Axin-1 induction to only 30 minutes of heat shock, and were able to show that CNS angiogenesis occurred without barriergenesis. This suggests that even though CNS angiogenesis and barriergenesis occur simultaneously, they do so via different mechanisms. Therefore, we proposed that barrier properties would not exist without angiogenesis in the brain as fundamental transplant experiments demonstrated the brain provides a microenvironment important for barrier function ${ }^{32}$. To our surprise, after treating 24 hpf $T g$ (glut1b:mCherry;flila:EGFP) embryos with a potent VEGFR2 antagonist, we saw that CNS angiogenesis did not occur but PHBCs that run alongside the hindbrain were still glut $1 b$ positive. This suggested that angiogenesis is not required for barrier properties to form and VEGF signaling is not required for barriergenesis.

We performed additional characterization studies with chemical inhibitors of Shh and BMP signaling as they have been studied in vascular development ${ }^{33,105}$. After trying to chemically inhibit Shh with Cyclopamine and BMP with DMH1, we saw no effect on glut $1 b$ expression. While Shh has been implicated in the mammalian BBB, this result suggests that these signals are not required for glut $1 b$ expression in zebrafish BECs. Genetic knockdown of these pathways could further and more systematically resolve their contribution to the zebrafish BBB.

The last hypothesis we wanted to examine during the characterization of $\operatorname{Tg}$ (glut1b:mCherry) was the role of shear stress on barrier properties. It has been demonstrated that the physical circulation of blood and perhaps factors within circulation, contribute to BBB properties $^{107}$. Because zebrafish can survive up to a week without a heartbeat, we decided to perform morpholino knockdown of tnnt $2 a$, a gene responsible for cardiac contractility in the zebrafish. Upon morpholino injection in our BBB line, there was no heartbeat but we were surprised to see that the glut $1 b$ signal was not diminished but possibly stronger in tnnt $2 a$ morphants. The BEC lumens looked collapsed compared to un-injected siblings and therefore may explain any increase in glut 1b:mCherry expression that we visualized.

Upon final characterization of our BBB reporter line and completion of the first specific aim, we utilized the $T g$ (glut1b:mCherry;flila:EGFP) line for a $\mathrm{F}_{3}$ forward genetic screen and chemical screen. For our genetic screen, adult male Tg(glut1b:mCherry;flila:EGFP)fish were treated with the carcinogenic ENU once a week for three weeks. Of the original males treated, 9 males survived and were used for creating the subsequent $\mathrm{F}_{1}$ generation. We were unable to perform a specific locus test with the nacre and roy loci to calculate an estimated ENU proficiency due to small clutch size and unwillingness of the $\mathrm{F}_{0}$ males to breed with Casper females. However, after fish 
recovered their first treatment, we tested the efficiency of ENU treatment by crossing treated males to untreated TL females. We saw that after only one round of treatment, the ENU concentration was highly effective due to a complete elimination of flila:EGFP expression in progeny from the founder male. We generated $625 \mathrm{~F}_{1}$ males and $210 \mathrm{~F}_{2}$ families. To date, of these 210 families 114 families survived and 68 families have been screened. From these 68 families, 4 BBB mutants have been identified, confirmed, and out-crossed to polymorphic strains for genetic studies. In families 40, 44, and 196, mutants were found that had little to no CNS angiogenesis but normal peripheral angiogenesis. Family 22 harbored our most interesting mutant, line 22.6, whereby there was still a degree of CNS angiogenesis that took place but a major reduction in glut 1b:mCherry expression. These 4 lines represent the first zebrafish BBB mutants produced from a forward genetic screen.

We wanted to follow-up and clone the 22.6 mutant to identify a potential novel regulator of barriergenesis. Positional cloning is commonly performed in the zebrafish with bulk segregant analysis PCR ${ }^{129}$. While this method has cloned zebrafish mutants, it is not fully guaranteed for every mutant identified in a genetic screen ${ }^{134}$. To increase our chances of cloning 22.6, we simultaneously performed exome sequencing on pools from 22.6 mutant and wild-type sibling genomic DNA. After bioinformatics analysis was performed in the Hartwell Center at St. Jude Children's Research Hospital, two candidate mutations were identified. Both candidate alleles were PCR amplified and sequenced from the original individual wild-type and 22.6 mutant samples. We confirmed that gpr 124 was the mutated allele in the 22.6 line, whereby a $\mathrm{G}$ to A point mutation causes a premature stop codon at 981 amino acids in the protein sequence. The premature stop codon was also stronger evidence for the mutation of gpr 124. Furthermore, sequencing revealed that the candidate $a d r a 2 b$ mutation was a polymorphism among wild-type siblings. While the first BBB mutant we cloned was in a gene known to be important for mammalian barriergenesis, this result confirmed our whole screening strategy and that we could faithfully identify BBB mutants ${ }^{35,126}$.

We next wanted to characterize the $\operatorname{gpr} 124^{-/-}$mutant to look for similarities and potential differences between our fish line and the mammalian knockout models. Gpr $124^{-/}$knockout mice present brain hemorrhages and die embryonically ${ }^{35,126}$. While we did not identify any brain hemorrhages in $\operatorname{gpr} 124^{-/-}$after it was out-crossed to TL, we did visualize that the gpr $124^{-/-}$barrier was "leakier" compared to wild-type siblings after IV injection with 3,000 Da Cascade Blue and $10 \mathrm{kDa}$ Rhodamine tracers. Mutants also appeared leakier when bathed in 332 Da Fluorescein dye. Because protein albumin is much larger than these tracers, this could suggest a difference in size-selectivity upon ablation of gpr 124 in zebrafish compared to mammals. We also looked at Claudin5 expression in $g$ pr $124^{-/-}$to identify if that was the cause for tracer leakage. Claudin $5^{-/-}$ mice also do not present brain hemorrhage but do show a size-selective loosening of the barrier like our $g$ pr $124^{-/}$when injected with tracer dyes ${ }^{22}$. It is hypothesized that the absence of brain hemorrhage in the zebrafish $\mathrm{gpr} 124^{-/-}$may also be due to compensation from another zebrafish g-coupled protein receptor paralog resulting from the zebrafish genome duplication event ${ }^{46}$. Ultimately, identification of the first zebrafish $g p r 124^{-/}$ mutant could lead to small molecule screens or future protein studies that identify the 
ligand for this orphan receptor. Identification of the gpr124 ligand could also prove useful as a therapeutic target in CNS drug delivery as many therapeutics target g-coupled proteins ${ }^{139}$. RNA-seq experiments with gpr $124^{-/ /}$and wild-type siblings may elucidate additional signals important for barriergenesis.

While we spawned the various generations for our $\mathrm{F}_{3}$ forward genetic screen, we simultaneously performed small molecule screens to identify chemical modulators of the BBB. To date, we have screened the FDA and ENZO Wnt, Autophagy, and Neurotransmitter libraries. From the 884 compounds in the FDA library, we identified our most conspicuous hit to be a class of compounds called statins. We found that depending on the statin, zebrafish treated at $24 \mathrm{hpf}$ developed CNS necrosis, brain hemorrhage and even death by $48 \mathrm{hpf}$ and had reduced CNS angiogenesis. Statins are known reducers of cholesterol biosynthesis, but recent zebrafish studies have shown that statins induce brain hemorrhage in zebrafish via mechanisms independent of cholesterol inhibition ${ }^{150,151}$. While our finding was not unique, our work from the FDA screen was confirmed by other groups. Furthermore, after performing several dose responses with the range of statins available, we found that based on the lipophilicity of the statin, zebrafish were a predictive indicator of BBB statin penetration. With a developmental treatment time course, we were also able to establish that statin treatment before zygotic transcription or later at $48 \mathrm{hpf}$ still caused brain hemorrhage and reduced CNS angiogenesis in the developing zebrafish. Brain hemorrhages were no longer seen in fish treated at $5 \mathrm{dpf}$, suggesting a developmental effect of statin treatment during barriergenesis. This raises concern for pregnant women with hypercholesterolemia, but the safety of statin treatment during pregnancy is dubious due to conflicting evidence and high treatment regimens in animal studies versus what is observed in humans ${ }^{159}$. Furthermore, signals important for barrier maturation may deviate from signals in differentiated BECs ${ }^{102}$.

While mechanisms have been suggested by other groups, we suggested that modification of N-linked glycosylation could be why statins cause brain hemorrhage. We suggest this because after treatment with Tunicamycin, a potent inhibitor of $\mathrm{N}$-linked glycosylation, zebrafish have reduced CNS angiogenesis and develop brain hemorrhages. Also, upon morpholino knockdown of dolk, the enzyme upstream Tunicamycin's target, we find that CNS angiogenesis is disrupted and a percentage of morphants develop brain hemorrhage. Even though we only saw at most $15 \%$ of morphants with brain hemorrhage, this could be because dolk is maternally derived and the morpholino diffuses in the animal over time. Future studies using techniques like CRISPR knockout of the dolk gene may be able to produce a more penetrant brain hemorrhage phenotype and study the consequence of true dolk knockout effects on the BBB. It would also be interesting to perform protein or RNA-seq studies on Tunicamycin-treated embryos to see if there are particular N-linked glycosylated proteins in the BBB that are affected after Tunicamycin treatment and cause the subsequent brain hemorrhage phenotype.

We identified two other chemical hits from the ENZO library screens, Forskolin and GBR13069. Forskolin a known elevator of cAMP signaling, has been indicated in enhancing TEER and reducing tight junction permeability ${ }^{157}$. Upon treatment with 
Forskolin, zebrafish had increased motor activity and a marked reduction in CNS angiogenesis. GBR13069 is a dopamine uptake inhibitor which has shown increased motor activity in mice ${ }^{153}$. After GBR13069 treatment, zebrafish had a discernible reduction in the $T g$ (glut 1 b:mCherry) signal. This reduction seemed to be hindbrain specific which is congruent with dopamine receptor expression in the zebrafish larvae ${ }^{154}$. Both of these compounds also remained effective with individual dose responses. These hits confirm the presence of cAMP signaling in the BBB as well as the first report on how neurons may specifically regulate barriergenesis.

The development of $\operatorname{Tg}$ (glut 1 : $m$ Cherry) helped elucidate questions about barriergenesis in a developing vertebrate that until now have not been possible. Glut1 is only one marker for the BBB and therefore it could be suggested that Glut cannot be the solitary indicator of barrier properties. However, this marker is supported by studies performed in mammals that use Glut 1 as a sole indicator of barrier integrity ${ }^{27,35}$. Additional zebrafish BBB transgenic lines should be generated to see if the results found from our studies with glut $1 b$ hold true with other markers. We demonstrate that our BBB line was useful for studying the onset of barriergenesis, pathways that modulate this process, the discovery of BBB mutants, and identification of compounds that may uncover novel regulators of barriergenesis. Therefore, the work in this dissertation will guide the understanding of the molecular mechanisms that help develop and maintain a healthy BBB. Recognizing these process prove to be crucial for furthering the development of CNS therapies, targets for better CNS drug penetration, and the prevention of CNS disease progression. 


\section{LIST OF REFERENCES}

1. Saunders, N. R., Liddelow, S. A. \& Dziegielewska, K. M. Barrier mechanisms in the developing brain. Frontiers in pharmacology 3, 46, doi:10.3389/fphar.2012.00046 (2012).

2. Zlokovic, B. V. The blood-brain barrier in health and chronic neurodegenerative disorders. Neuron 57, 178-201, doi:10.1016/j.neuron.2008.01.003 (2008).

3. Saunders, N. R., Ek, C. J., Habgood, M. D. \& Dziegielewska, K. M. Barriers in the brain: a renaissance? Trends in neurosciences 31, 279-286, doi:10.1016/j.tins.2008.03.003 (2008).

4. Bell, R. D. et al. Pericytes control key neurovascular functions and neuronal phenotype in the adult brain and during brain aging. Neuron 68, 409-427, doi:10.1016/j.neuron.2010.09.043 (2010).

5. Pardridge, W. M. The blood-brain barrier: bottleneck in brain drug development. NeuroRx: the journal of the American Society for Experimental NeuroTherapeutics 2, 3-14, doi:10.1602/neurorx.2.1.3 (2005).

6. Pardridge, W. M. Drug delivery to the brain. Journal of cerebral blood flow and metabolism : official journal of the International Society of Cerebral Blood Flow and Metabolism 17, 713-731, doi:10.1097/00004647-199707000-00001 (1997).

7. Loscher, W. \& Potschka, H. Drug resistance in brain diseases and the role of drug efflux transporters. Nature reviews. Neuroscience 6, 591-602, doi:10.1038/nrn1728 (2005).

8. Cordon-Cardo, C. et al. Multidrug-resistance gene (P-glycoprotein) is expressed by endothelial cells at blood-brain barrier sites. Proc Natl Acad Sci U S A 86, 695698 (1989).

9. Thiebaut, F. et al. Immunohistochemical localization in normal tissues of different epitopes in the multidrug transport protein P170: evidence for localization in brain capillaries and crossreactivity of one antibody with a muscle protein. The journal of histochemistry and cytochemistry : official journal of the Histochemistry Society 37, 159-164 (1989).

10. Wohlfart, S., Gelperina, S. \& Kreuter, J. Transport of drugs across the blood-brain barrier by nanoparticles. Journal of controlled release : official journal of the Controlled Release Society 161, 264-273, doi:10.1016/j.jconrel.2011.08.017 (2012).

11. Begley, D. J. Delivery of therapeutic agents to the central nervous system: the problems and the possibilities. Pharmacology \& therapeutics 104, 29-45, doi:10.1016/j.pharmthera.2004.08.001 (2004).

12. Breedveld, P., Beijnen, J. H. \& Schellens, J. H. Use of P-glycoprotein and BCRP inhibitors to improve oral bioavailability and CNS penetration of anticancer drugs. Trends in pharmacological sciences 27, 17-24, doi:10.1016/j.tips.2005.11.009 (2006).

13. Kinoshita, M., McDannold, N., Jolesz, F. A. \& Hynynen, K. Targeted delivery of antibodies through the blood-brain barrier by MRI-guided focused ultrasound.

Biochemical and biophysical research communications 340, 1085-1090, doi:10.1016/j.bbrc.2005.12.112 (2006). 
14. Hynynen, K., McDannold, N., Sheikov, N. A., Jolesz, F. A. \& Vykhodtseva, N. Local and reversible blood-brain barrier disruption by noninvasive focused ultrasound at frequencies suitable for trans-skull sonications. NeuroImage 24, 1220, doi:10.1016/j.neuroimage.2004.06.046 (2005).

15. Gabathuler, R. Approaches to transport therapeutic drugs across the blood-brain barrier to treat brain diseases. Neurobiology of disease 37, 48-57, doi:10.1016/j.nbd.2009.07.028 (2010).

16. Pardridge, W. M. Blood-brain barrier delivery. Drug discovery today 12, 54-61, doi:10.1016/j.drudis.2006.10.013 (2007).

17. Ehrlich, P. Das sauerstoff-bedürfnis des organismus, in Eine Farbenanalytische Studie, (1885).

18. Ehrlich, P. Ueber die beziehungen von chemischer constitution, vertheilung, und pharmakologischen wirkung. (J. Wiley \& Sons, 1906).

19. Goldmann, E. E. Vitalfärbung am zentralnervensystem. Abhandl Königl Preuss Akad Wiss, 1-60 (1913).

20. Engelhardt, B. Development of the blood-brain barrier. Cell and tissue research 314, 119-129, doi:10.1007/s00441-003-0751-z (2003).

21. Brightman, M. W. \& Reese, T. S. Junctions between intimately apposed cell membranes in the vertebrate brain. The Journal of cell biology 40, 648-677 (1969).

22. Nitta, T. et al. Size-selective loosening of the blood-brain barrier in claudin-5deficient mice. The Journal of cell biology 161, 653-660, doi:10.1083/jcb.200302070 (2003).

23. Abbott, N. J., Ronnback, L. \& Hansson, E. Astrocyte-endothelial interactions at the blood-brain barrier. Nature reviews. Neuroscience 7, 41-53, doi:10.1038/nrn1824 (2006).

24. Schinkel, A. H. et al. Disruption of the mouse mdr1a P-glycoprotein gene leads to a deficiency in the blood-brain barrier and to increased sensitivity to drugs. Cell 77, 491-502 (1994).

25. Lee, G., Dallas, S., Hong, M. \& Bendayan, R. Drug transporters in the central nervous system: brain barriers and brain parenchyma considerations. Pharmacological reviews 53, 569-596 (2001).

26. Klepper, J. \& Voit, T. Facilitated glucose transporter protein type 1 (GLUT1) deficiency syndrome: impaired glucose transport into brain-- a review. European journal of pediatrics 161, 295-304, doi:10.1007/s00431-002-0939-3 (2002).

27. Daneman, R., Zhou, L., Kebede, A. A. \& Barres, B. A. Pericytes are required for blood-brain barrier integrity during embryogenesis. Nature 468, 562-566, doi:10.1038/nature09513 (2010).

28. Dermietzel, R., Krause, D., Kremer, M., Wang, C. \& Stevenson, B. Pattern of glucose transporter (Glut 1) expression in embryonic brains is related to maturation of blood-brain barrier tightness. Developmental dynamics : an official publication of the American Association of Anatomists 193, 152-163, doi:10.1002/aja.1001930207 (1992). 
29. Hellstrom, M., Kalen, M., Lindahl, P., Abramsson, A. \& Betsholtz, C. Role of PDGF-B and PDGFR-beta in recruitment of vascular smooth muscle cells and pericytes during embryonic blood vessel formation in the mouse. Development 126, 3047-3055 (1999).

30. Janzer, R. C. \& Raff, M. C. Astrocytes induce blood-brain barrier properties in endothelial cells. Nature 325, 253-257, doi:10.1038/325253a0 (1987).

31. Stenman, J. M. et al. Canonical Wnt signaling regulates organ-specific assembly and differentiation of CNS vasculature. Science 322, 1247-1250, doi:10.1126/science.1164594 (2008).

32. Stewart, P. A. \& Wiley, M. J. Developing nervous tissue induces formation of blood-brain barrier characteristics in invading endothelial cells: a study using quail--chick transplantation chimeras. Developmental biology 84, 183-192 (1981).

33. Alvarez, J. I. et al. The Hedgehog pathway promotes blood-brain barrier integrity and CNS immune quiescence. Science 334, 1727-1731, doi:10.1126/science.1206936 (2011).

34. Kuhnert, F. et al. Essential regulation of CNS angiogenesis by the orphan $\mathrm{G}$ protein-coupled receptor GPR124. Science 330, 985-989, doi:10.1126/science.1196554 (2010).

35. Cullen, M. et al. GPR124, an orphan G protein-coupled receptor, is required for CNS-specific vascularization and establishment of the blood-brain barrier. Proc Natl Acad Sci U S A 108, 5759-5764, doi:10.1073/pnas.1017192108 (2011).

36. Daneman, R. et al. Wnt/beta-catenin signaling is required for CNS, but not nonCNS, angiogenesis. Proc Natl Acad Sci U S A 106, 641-646, doi:0805165106 [pii] 10.1073/pnas.0805165106 (2009).

37. Salinas, P. C. \& Zou, Y. Wnt signaling in neural circuit assembly. Annual review of neuroscience 31, 339-358, doi:10.1146/annurev.neuro.31.060407.125649 (2008).

38. Ruiz i Altaba, A., Palma, V. \& Dahmane, N. Hedgehog-Gli signalling and the growth of the brain. Nature reviews. Neuroscience 3, 24-33, doi:10.1038/nrn704 (2002).

39. Daneman, R. et al. The mouse blood-brain barrier transcriptome: a new resource for understanding the development and function of brain endothelial cells. PloS one 5, e13741, doi:10.1371/journal.pone.0013741 (2010).

40. Ben-Zvi, A. et al. Mfsd2a is critical for the formation and function of the bloodbrain barrier. Nature 509, 507-511, doi:10.1038/nature13324 (2014).

41. Armulik, A. et al. Pericytes regulate the blood-brain barrier. Nature 468, 557-561, doi:10.1038/nature09522 (2010).

42. Geldenhuys, W. J., Allen, D. D. \& Bloomquist, J. R. Novel models for assessing blood-brain barrier drug permeation. Expert opinion on drug metabolism \& toxicology 8, 647-653, doi:10.1517/17425255.2012.677433 (2012).

43. Mayer, F. et al. Evolutionary conservation of vertebrate blood-brain barrier chemoprotective mechanisms in Drosophila. The Journal of neuroscience : the official journal of the Society for Neuroscience 29, 3538-3550, doi:10.1523/JNEUROSCI.5564-08.2009 (2009).

44. Wilhelm, I., Fazakas, C. \& Krizbai, I. A. In vitro models of the blood-brain barrier. Acta neurobiologiae experimentalis 71, 113-128 (2011). 
45. Lippmann, E. S., Weidenfeller, C., Svendsen, C. N. \& Shusta, E. V. Blood-brain barrier modeling with co-cultured neural progenitor cell-derived astrocytes and neurons. Journal of neurochemistry 119, 507-520, doi:10.1111/j.14714159.2011.07434.x (2011).

46. Amores, A. et al. Zebrafish hox clusters and vertebrate genome evolution. Science 282, 1711-1714 (1998).

47. Lieschke, G. J. \& Currie, P. D. Animal models of human disease: zebrafish swim into view. Nature reviews. Genetics 8, 353-367, doi:10.1038/nrg2091 (2007).

48. Driever, W. et al. A genetic screen for mutations affecting embryogenesis in zebrafish. Development 123, 37-46 (1996).

49. Haffter, P. et al. The identification of genes with unique and essential functions in the development of the zebrafish, Danio rerio. Development 123, 1-36 (1996).

50. Muto, A. et al. Forward genetic analysis of visual behavior in zebrafish. PLoS genetics 1, e66, doi:10.1371/journal.pgen.0010066 (2005).

51. Shepard, J. L. et al. A zebrafish bmyb mutation causes genome instability and increased cancer susceptibility. Proc Natl Acad Sci U S A 102, 13194-13199, doi:10.1073/pnas.0506583102 (2005).

52. Habeck, H., Odenthal, J., Walderich, B., Maischein, H. \& Schulte-Merker, S. Analysis of a zebrafish VEGF receptor mutant reveals specific disruption of angiogenesis. Current biology : CB 12, 1405-1412 (2002).

53. Lawson, N. D. \& Wolfe, S. A. Forward and reverse genetic approaches for the analysis of vertebrate development in the zebrafish. Developmental cell 21, 48-64, doi:10.1016/j.devcel.2011.06.007 (2011).

54. Huang, P. et al. Heritable gene targeting in zebrafish using customized TALENs. Nature biotechnology 29, 699-700, doi:10.1038/nbt.1939 (2011).

55. Hwang, W. Y. et al. Efficient genome editing in zebrafish using a CRISPR-Cas system. Nature biotechnology 31, 227-229, doi:10.1038/nbt.2501 (2013).

56. Ceol, C. J. et al. The histone methyltransferase SETDB1 is recurrently amplified in melanoma and accelerates its onset. Nature 471, 513-517, doi:10.1038/nature09806 (2011).

57. Zon, L. I. \& Peterson, R. T. In vivo drug discovery in the zebrafish. Nature reviews. Drug discovery 4, 35-44, doi:10.1038/nrd1606 (2005).

58. Yu, P. B. et al. Dorsomorphin inhibits BMP signals required for embryogenesis and iron metabolism. Nature chemical biology 4, 33-41, doi:10.1038/nchembio.2007.54 (2008).

59. Stern, H. M. et al. Small molecules that delay S phase suppress a zebrafish bmyb mutant. Nature chemical biology 1, 366-370 (2005).

60. Wang, C. et al. Rosuvastatin, identified from a zebrafish chemical genetic screen for antiangiogenic compounds, suppresses the growth of prostate cancer.

European urology 58, 418-426, doi:10.1016/j.eururo.2010.05.024 (2010).

61. Jeong, J. Y. et al. Functional and developmental analysis of the blood-brain barrier in zebrafish. Brain research bulletin 75, 619-628, doi:10.1016/j.brainresbull.2007.10.043 (2008).

62. Xie, J., Farage, E., Sugimoto, M. \& Anand-Apte, B. A novel transgenic zebrafish model for blood-brain and blood-retinal barrier development. BMC developmental biology 10, 76, doi:10.1186/1471-213X-10-76 (2010). 
63. Tseng, Y. C. et al. Specific expression and regulation of glucose transporters in zebrafish ionocytes. American journal of physiology. Regulatory, integrative and comparative physiology 297, R275-290, doi:10.1152/ajpregu.00180.2009 (2009).

64. Tam, S. J. et al. Death receptors DR6 and TROY regulate brain vascular development. Developmental cell 22, 403-417, doi:10.1016/j.devcel.2011.11.018 (2012).

65. Umans, R. A. \& Taylor, M. R. Zebrafish as a model to study drug transporters at the blood-brain barrier. Clin Pharmacol Ther 92, 567-570, doi:10.1038/clpt.2012.168 (2012).

66. Fischer, S. et al. Abcb4 acts as multixenobiotic transporter and active barrier against chemical uptake in zebrafish (Danio rerio) embryos. BMC biology 11, 69, doi:10.1186/1741-7007-11-69 (2013).

67. Abdelilah-Seyfried, S. Claudin-5a in developing zebrafish brain barriers: another brick in the wall. BioEssays : news and reviews in molecular, cellular and developmental biology 32, 768-776, doi:10.1002/bies.201000045 (2010).

68. Zhang, J. et al. Establishment of a neuroepithelial barrier by Claudin5a is essential for zebrafish brain ventricular lumen expansion. Proc Natl Acad Sci U S A 107, 1425-1430, doi:10.1073/pnas.0911996107 (2010).

69. Daneman, R. \& Rescigno, M. The gut immune barrier and the blood-brain barrier: are they so different? Immunity 31, 722-735, doi:10.1016/j.immuni.2009.09.012 (2009).

70. Isogai, S., Horiguchi, M. \& Weinstein, B. M. The vascular anatomy of the developing zebrafish: an atlas of embryonic and early larval development. Developmental biology 230, 278-301, doi:10.1006/dbio.2000.9995 (2001).

71. Gale, N. W. \& Yancopoulos, G. D. Growth factors acting via endothelial cellspecific receptor tyrosine kinases: VEGFs, angiopoietins, and ephrins in vascular development. Genes \& development 13, 1055-1066 (1999).

72. Carmeliet, P. et al. Abnormal blood vessel development and lethality in embryos lacking a single VEGF allele. Nature 380, 435-439, doi:10.1038/380435a0 (1996).

73. Covassin, L. D., Villefranc, J. A., Kacergis, M. C., Weinstein, B. M. \& Lawson, N. D. Distinct genetic interactions between multiple Vegf receptors are required for development of different blood vessel types in zebrafish. Proc Natl Acad Sci U S A 103, 6554-6559, doi:10.1073/pnas.0506886103 (2006).

74. Risau, W. Mechanisms of angiogenesis. Nature 386, 671-674, doi:10.1038/386671a0 (1997).

75. Bauer, H. et al. Ontogenic expression of the erythroid-type glucose transporter (Glut 1) in the telencephalon of the mouse: correlation to the tightening of the blood-brain barrier. Brain research. Developmental brain research 86, 317-325 (1995).

76. Wang, Y., Pan, L., Moens, C. B. \& Appel, B. Notch3 establishes brain vascular integrity by regulating pericyte number. Development 141, 307-317, doi:10.1242/dev.096107 (2014).

77. Bernardos, R. L. \& Raymond, P. A. GFAP transgenic zebrafish. Gene expression patterns : GEP 6, 1007-1013, doi:10.1016/j.modgep.2006.04.006 (2006). 
78. Molofsky, A. V. et al. Astrocytes and disease: a neurodevelopmental perspective. Genes \& development 26, 891-907, doi:10.1101/gad.188326.112 (2012).

79. Li, F. et al. Endothelial Smad4 maintains cerebrovascular integrity by activating N-cadherin through cooperation with Notch. Developmental cell 20, 291-302, doi:10.1016/j.devcel.2011.01.011 (2011).

80. Lawson, N. D. \& Weinstein, B. M. In vivo imaging of embryonic vascular development using transgenic zebrafish. Developmental biology 248, 307-318 (2002).

81. Hanahan, D., Wagner, E. F. \& Palmiter, R. D. The origins of oncomice: a history of the first transgenic mice genetically engineered to develop cancer. Genes \& development 21, 2258-2270, doi:10.1101/gad.1583307 (2007).

82. Schlaeger, T. M., Qin, Y., Fujiwara, Y., Magram, J. \& Sato, T. N. Vascular endothelial cell lineage-specific promoter in transgenic mice. Development 121, 1089-1098 (1995).

83. Fleming, A., Diekmann, H. \& Goldsmith, P. Functional characterisation of the maturation of the blood-brain barrier in larval zebrafish. PloS one 8, e77548, doi:10.1371/journal.pone.0077548 (2013).

84. Kwan, K. M. et al. The Tol2kit: a multisite gateway-based construction kit for Tol2 transposon transgenesis constructs. Developmental dynamics : an official publication of the American Association of Anatomists 236, 3088-3099, doi:10.1002/dvdy.21343 (2007).

85. Koga, A., Suzuki, M., Inagaki, H., Bessho, Y. \& Hori, H. Transposable element in fish. Nature 383, 30, doi:10.1038/383030a0 (1996).

86. Kawakami, K., Shima, A. \& Kawakami, N. Identification of a functional transposase of the Tol2 element, an Ac-like element from the Japanese medaka fish, and its transposition in the zebrafish germ lineage. Proc Natl Acad Sci U S A 97, 11403-11408, doi:10.1073/pnas.97.21.11403 (2000).

87. Kawakami, K. et al. A transposon-mediated gene trap approach identifies developmentally regulated genes in zebrafish. Developmental cell 7, 133-144, doi:10.1016/j.devcel.2004.06.005 (2004).

88. Pardridge, W. M. Molecular biology of the blood-brain barrier. Molecular biotechnology 30, 57-70, doi:10.1385/MB:30:1:057 (2005).

89. Shaner, N. C. et al. Improved monomeric red, orange and yellow fluorescent proteins derived from Discosoma sp. red fluorescent protein. Nature biotechnology 22, 1567-1572, doi:10.1038/nbt1037 (2004).

90. Nishimoto, H. et al. Gene expression of glucose transporter (GLUT) 1, 3 and 4 in bovine follicle and corpus luteum. The Journal of endocrinology 188, 111-119, doi:10.1677/joe.1.06210 (2006).

91. Linden, K. C. et al. Renal expression and localization of the facilitative glucose transporters GLUT1 and GLUT12 in animal models of hypertension and diabetic nephropathy. American journal of physiology. Renal physiology 290, F205-213, doi:10.1152/ajprenal.00237.2004 (2006).

92. Daneman, R. The blood-brain barrier in health and disease. Annals of neurology 72, 648-672, doi:10.1002/ana.23648 (2012). 
93. Shue, E. H. et al. Plasmalemmal vesicle associated protein-1 (PV-1) is a marker of blood-brain barrier disruption in rodent models. BMC neuroscience 9, 29, doi:10.1186/1471-2202-9-29 (2008).

94. Schulzke, J. D. et al. Epithelial transport and barrier function in occludin-deficient mice. Biochimica et biophysica acta 1669, 34-42, doi:10.1016/j.bbamem.2005.01.008 (2005).

95. Vlaming, M. L., Lagas, J. S. \& Schinkel, A. H. Physiological and pharmacological roles of ABCG2 (BCRP): recent findings in Abcg2 knockout mice. Advanced drug delivery reviews 61, 14-25, doi:10.1016/j.addr.2008.08.007 (2009).

96. Schinkel, A. H. P-Glycoprotein, a gatekeeper in the blood-brain barrier. Advanced drug delivery reviews 36, 179-194 (1999).

97. Jaffer, H., Adjei, I. M. \& Labhasetwar, V. Optical imaging to map blood-brain barrier leakage. Scientific reports 3, 3117, doi:10.1038/srep03117 (2013).

98. Sehnert, A. J. et al. Cardiac troponin T is essential in sarcomere assembly and cardiac contractility. Nature genetics 31, 106-110, doi:10.1038/ng875 (2002).

99. Bill, B. R. et al. Development and Notch signaling requirements of the zebrafish choroid plexus. PloS one 3, e3114, doi:10.1371/journal.pone.0003114 (2008).

100. Garcia-Lecea, M., Kondrychyn, I., Fong, S. H., Ye, Z. R. \& Korzh, V. In vivo analysis of choroid plexus morphogenesis in zebrafish. PloS one 3, e3090, doi:10.1371/journal.pone.0003090 (2008).

101. Dejana, E. The role of wnt signaling in physiological and pathological angiogenesis. Circulation research 107, 943-952, doi:10.1161/CIRCRESAHA.110.223750 (2010).

102. Liebner, S. et al. Wnt/beta-catenin signaling controls development of the bloodbrain barrier. The Journal of cell biology 183, 409-417, doi:10.1083/jcb.200806024 (2008).

103. Wang, X. et al. Wnt signaling regulates postembryonic hypothalamic progenitor differentiation. Developmental cell 23, 624-636, doi:10.1016/j.devcel.2012.07.012 (2012).

104. Chimote, G. et al. Comparison of effects of anti-angiogenic agents in the zebrafish efficacy-toxicity model for translational anti-angiogenic drug discovery. Drug design, development and therapy 8, 1107-1123, doi:10.2147/DDDT.S55621 (2014).

105. Hao, J. et al. In vivo structure-activity relationship study of dorsomorphin analogues identifies selective VEGF and BMP inhibitors. ACS chemical biology 5, 245-253, doi:10.1021/cb9002865 (2010).

106. Coultas, L., Chawengsaksophak, K. \& Rossant, J. Endothelial cells and VEGF in vascular development. Nature 438, 937-945, doi:10.1038/nature04479 (2005).

107. Cucullo, L., Hossain, M., Puvenna, V., Marchi, N. \& Janigro, D. The role of shear stress in Blood-Brain Barrier endothelial physiology. BMC neuroscience 12, 40, doi:10.1186/1471-2202-12-40 (2011).

108. Fujita, M. et al. Assembly and patterning of the vascular network of the vertebrate hindbrain. Development 138, 1705-1715, doi:10.1242/dev.058776 (2011).

109. Patton, E. E. \& Zon, L. I. The art and design of genetic screens: zebrafish. Nature reviews. Genetics 2, 956-966, doi:10.1038/35103567 (2001). 
110. Streisinger, G., Walker, C., Dower, N., Knauber, D. \& Singer, F. Production of clones of homozygous diploid zebra fish (Brachydanio rerio). Nature 291, 293296 (1981).

111. Brenner, S. The genetics of Caenorhabditis elegans. Genetics 77, 71-94 (1974).

112. Nusslein-Volhard, C. \& Wieschaus, E. Mutations affecting segment number and polarity in Drosophila. Nature 287, 795-801 (1980).

113. Chakrabarti, S., Streisinger, G., Singer, F. \& Walker, C. Frequency of gammaRay Induced Specific Locus and Recessive Lethal Mutations in Mature Germ Cells of the Zebrafish, BRACHYDANIO RERIO. Genetics 103, 109-123 (1983).

114. Walker, C. \& Streisinger, G. Induction of Mutations by gamma-Rays in Pregonial Germ Cells of Zebrafish Embryos. Genetics 103, 125-136 (1983).

115. Brockerhoff, S. E. et al. A behavioral screen for isolating zebrafish mutants with visual system defects. Proc Natl Acad Sci U S A 92, 10545-10549 (1995).

116. Stainier, D. Y. et al. Mutations affecting the formation and function of the cardiovascular system in the zebrafish embryo. Development 123, 285-292 (1996).

117. Schier, A. F. et al. Mutations affecting the development of the embryonic zebrafish brain. Development 123, 165-178 (1996).

118. Trevarrow, B. Techniques for optimizing the creation of mutations in zebrafish using N-ethyl-N-nitrosourea. Lab animal 40, 353-361, doi:10.1038/laban1111353 (2011).

119. Noveroske, J. K., Weber, J. S. \& Justice, M. J. The mutagenic action of N-ethyl$\mathrm{N}$-nitrosourea in the mouse. Mammalian genome : official journal of the International Mammalian Genome Society 11, 478-483 (2000).

120. Solnica-Krezel, L., Schier, A. F. \& Driever, W. Efficient recovery of ENUinduced mutations from the zebrafish germline. Genetics 136, 1401-1420 (1994).

121. Mullins, M. C., Hammerschmidt, M., Haffter, P. \& Nusslein-Volhard, C. Largescale mutagenesis in the zebrafish: in search of genes controlling development in a vertebrate. Current biology : CB 4, 189-202 (1994).

122. Salinger, A. P. \& Justice, M. J. Mouse Mutagenesis Using N-Ethyl-N-Nitrosourea (ENU). CSH protocols 2008, pdb prot4985, doi:10.1101/pdb.prot4985 (2008).

123. de Bruijn, E., Cuppen, E., \& Feitsma, H. . in Zebrafish: Methods and Protocols. Methods in Molecular Biology Vol. 546 Methods in Molecular Biology (ed G.J Lieschke, Oates, A.C., \& Kawakami, K. ) Ch. 1, 1-12 (Humana Press, 2009).

124. Buchner, D. A. et al. pak2a mutations cause cerebral hemorrhage in redhead zebrafish. Proc Natl Acad Sci U S A 104, 13996-14001, doi:10.1073/pnas.0700947104 (2007).

125. White, R. M. et al. Transparent adult zebrafish as a tool for in vivo transplantation analysis. Cell stem cell 2, 183-189, doi:10.1016/j.stem.2007.11.002 (2008).

126. Anderson, K. D. et al. Angiogenic sprouting into neural tissue requires Gpr124, an orphan G protein-coupled receptor. Proc Natl Acad Sci U S A 108, 2807-2812, doi:10.1073/pnas.1019761108 (2011).

127. Knapik, E. W. et al. A microsatellite genetic linkage map for zebrafish (Danio rerio). Nature genetics 18, 338-343, doi:10.1038/ng0498-338 (1998).

128. Postlethwait, J. H. et al. A genetic linkage map for the zebrafish. Science 264, 699-703 (1994). 
129. Talbot, W. S. S., A.F. . in Essential Zebrafish Methods: Genetics and Genomics (ed H.W. Detrich III, Westerfield, M., \& Zon, L.I.) Ch. 2, 30-44 (Academic Press, 2009).

130. Henke, K., Bowen, M. E. \& Harris, M. P. Perspectives for identification of mutations in the zebrafish: making use of next-generation sequencing technologies for forward genetic approaches. Methods 62, 185-196, doi:10.1016/j.ymeth.2013.05.015 (2013).

131. Donovan, A. et al. Positional cloning of zebrafish ferroportin1 identifies a conserved vertebrate iron exporter. Nature 403, 776-781, doi:10.1038/35001596 (2000).

132. Zhang, J., Talbot, W. S. \& Schier, A. F. Positional cloning identifies zebrafish one-eyed pinhead as a permissive EGF-related ligand required during gastrulation. Cell 92, 241-251 (1998).

133. Lamason, R. L. et al. SLC24A5, a putative cation exchanger, affects pigmentation in zebrafish and humans. Science 310, 1782-1786, doi:10.1126/science.1116238 (2005).

134. del Viso, F., Bhattacharya, D., Kong, Y., Gilchrist, M. J. \& Khokha, M. K. Exon capture and bulk segregant analysis: rapid discovery of causative mutations using high-throughput sequencing. BMC genomics 13, 649, doi:10.1186/1471-2164-13649 (2012).

135. Kettleborough, R. N. et al. A systematic genome-wide analysis of zebrafish protein-coding gene function. Nature 496, 494-497, doi:10.1038/nature11992 (2013).

136. Voz, M. L. et al. Fast homozygosity mapping and identification of a zebrafish ENU-induced mutation by whole-genome sequencing. PloS one 7, e34671, doi:10.1371/journal.pone.0034671 (2012).

137. Obholzer, N. et al. Rapid positional cloning of zebrafish mutations by linkage and homozygosity mapping using whole-genome sequencing. Development 139, 4280-4290, doi:10.1242/dev.083931 (2012).

138. Schneeberger, K. Using next-generation sequencing to isolate mutant genes from forward genetic screens. Nature reviews. Genetics, doi:10.1038/nrg3745 (2014).

139. Lappano, R. \& Maggiolini, M. G protein-coupled receptors: novel targets for drug discovery in cancer. Nature reviews. Drug discovery 10, 47-60, doi:10.1038/nrd3320 (2011).

140. Hughes, J. P., Rees, S., Kalindjian, S. B. \& Philpott, K. L. Principles of early drug discovery. British journal of pharmacology 162, 1239-1249, doi:10.1111/j.14765381.2010.01127.x (2011).

141. Peterson, J. R. \& Mitchison, T. J. Small molecules, big impact: a history of chemical inhibitors and the cytoskeleton. Chemistry \& biology 9, 1275-1285 (2002).

142. Peterson, R. T. \& Fishman, M. C. Designing zebrafish chemical screens. Methods in cell biology 105, 525-541, doi:10.1016/B978-0-12-381320-6.00023-0 (2011).

143. Eggert, U. S. The why and how of phenotypic small-molecule screens. Nature chemical biology 9, 206-209, doi:10.1038/nchembio.1206 (2013). 
144. Kitambi, S. S., McCulloch, K. J., Peterson, R. T. \& Malicki, J. J. Small molecule screen for compounds that affect vascular development in the zebrafish retina. Mechanisms of development 126, 464-477 (2009).

145. Huang, H., Lindgren, A., Wu, X., Liu, N. A. \& Lin, S. High-throughput screening for bioactive molecules using primary cell culture of transgenic zebrafish embryos. Cell reports 2, 695-704, doi:10.1016/j.celrep.2012.08.015 (2012).

146. Sachidanandan, C., Yeh, J. R., Peterson, Q. P. \& Peterson, R. T. Identification of a novel retinoid by small molecule screening with zebrafish embryos. PloS one $\mathbf{3}$, e1947, doi:10.1371/journal.pone.0001947 (2008).

147. Ren, B. et al. ERK1/2-Akt1 crosstalk regulates arteriogenesis in mice and zebrafish. The Journal of clinical investigation 120, 1217-1228, doi:10.1172/JCI39837 (2010).

148. Bellosta, S., Paoletti, R. \& Corsini, A. Safety of statins: focus on clinical pharmacokinetics and drug interactions. Circulation 109, III50-57, doi:10.1161/01.CIR.0000131519.15067.1f(2004).

149. Malfitano, A. M. et al. Statins in neurological disorders: An overview and update. Pharmacological research : the official journal of the Italian Pharmacological Society, doi:10.1016/j.phrs.2014.06.007 (2014).

150. Mugoni, V. et al. Ubiad1 is an antioxidant enzyme that regulates eNOS activity by CoQ10 synthesis. Cell 152, 504-518, doi:10.1016/j.cell.2013.01.013 (2013).

151. Eisa-Beygi, S., Hatch, G., Noble, S., Ekker, M. \& Moon, T. W. The 3-hydroxy-3methylglutaryl-CoA reductase (HMGCR) pathway regulates developmental cerebral-vascular stability via prenylation-dependent signalling pathway. Developmental biology 373, 258-266, doi:10.1016/j.ydbio.2012.11.024 (2013).

152. Baraban, S. C., Taylor, M. R., Castro, P. A. \& Baier, H. Pentylenetetrazole induced changes in zebrafish behavior, neural activity and c-fos expression. Neuroscience 131, 759-768, doi:10.1016/j.neuroscience.2004.11.031 (2005).

153. Heikkila, R. E. \& Manzino, L. Behavioral properties of GBR 12909, GBR 13069 and GBR 13098: specific inhibitors of dopamine uptake. European journal of pharmacology 103, 241-248 (1984).

154. Boehmler, W. et al. Evolution and expression of D2 and D3 dopamine receptor genes in zebrafish. Developmental dynamics : an official publication of the American Association of Anatomists 230, 481-493, doi:10.1002/dvdy.20075 (2004).

155. Yamamoto, M. et al. Phosphorylation of claudin-5 and occludin by rho kinase in brain endothelial cells. The American journal of pathology 172, 521-533, doi:10.2353/ajpath.2008.070076 (2008).

156. Corsini, A. et al. New insights into the pharmacodynamic and pharmacokinetic properties of statins. Pharmacology \& therapeutics 84, 413-428 (1999).

157. Wolburg, H. et al. Modulation of tight junction structure in blood-brain barrier endothelial cells. Effects of tissue culture, second messengers and cocultured astrocytes. Journal of cell science 107 ( Pt 5), 1347-1357 (1994).

158. Ishizaki, T. et al. Cyclic AMP induces phosphorylation of claudin-5 immunoprecipitates and expression of claudin-5 gene in blood-brain-barrier endothelial cells via protein kinase A-dependent and -independent pathways. Experimental cell research 290, 275-288 (2003). 
159. Kusters, D. M. et al. Statin use during pregnancy: a systematic review and metaanalysis. Expert review of cardiovascular therapy 10, 363-378, doi:10.1586/erc.11.196 (2012). 


\section{VITA}

Robyn Anne Umans was born in 1987 in Freeport, Long Island, New York. After graduating from the University of Evansville in Evansville, Indiana with a Bachelor's of Science in Neuroscience in 2009, she continued her graduate studies with the Integrated Program of Biomedical Sciences at the University of Tennessee Health Science Center in Memphis, Tennessee in the fall of 2009. During her time in graduate school, Robyn held many leadership positions such as her college Honor Council President and Graduate Student Executive Committee President. Robyn also received several travel awards from St. Jude Children's Research Hospital, the University of Tennessee Health Science Center, and the Genetics Society of America to attend various conferences related to her dissertation work. She was also a scientific collaborator in several local Art of Science shows, which paired a scientist at St. Jude Children's Research Hospital with local Memphian artists. Her passion for scientific imaging was also displayed with a finalist image in the High Content Screening category of the 2013 GE Healthcare Imaging Competition. After final approval of her electronic dissertation, Robyn will receive a Doctorate of Philosophy with a major in Biomedical Sciences and a concentration in Neuroscience. She will graduate December 2014 from the University of Tennessee Health Science Center. 